\title{
Mercuric Triflate-Catalyzed Reaction of Propargyl Acetates with Water Leading Vinyl Ketones
}

Hiroshi Imagawa, Yumiko Asai, Hiroto Takano, Hitomi Hamagaki, Mugio Nishizawa*

Faculty of Pharmaceutical Sciences, Tokushima Bunri University, Yamashiro-cho,

Tokushima 770-8514, Japan

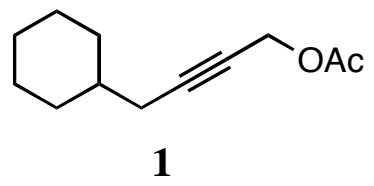

4-cyclohexylbut-2-ynyl acetate (1): FTIR 3473, 2931, 2852, 2307, 2237, 1757, 1378 $\mathrm{cm}^{-1} ;{ }^{1} \mathrm{H}$ NMR (300 MHz in $\mathrm{CDCl}_{3}$ ) $\delta$ 0.90-1.04 (2H, m), 1.06-1.31 (3H, m), 1.40-1.54 $(1 \mathrm{H}, \mathrm{m}), 1.62-1.83(5 \mathrm{H}, \mathrm{m}), 2.10(3 \mathrm{H}, \mathrm{s}), 2.11(2 \mathrm{H}, \mathrm{dt}, J=6.7,2.2 \mathrm{~Hz}), 4.67(2 \mathrm{H}, \mathrm{t}, J=$ $2.2 \mathrm{~Hz}) ;{ }^{13} \mathrm{C}$ NMR $\left(75 \mathrm{MHz}\right.$ in $\left.\mathrm{CDCl}_{3}\right) \delta 20.49 \mathrm{q}, 25.89 \mathrm{t}, 25.89 \mathrm{t}, 26.02 \mathrm{t}, 26.31 \mathrm{t}, 32.45 \mathrm{t}$, 32.45t, 36.93d, 52.58t, 74.62s, 86.18s, 169.98s; MS (CI) m/z $195\left(\mathrm{M}^{+}+\mathrm{H}\right)$; HRMS (CI)

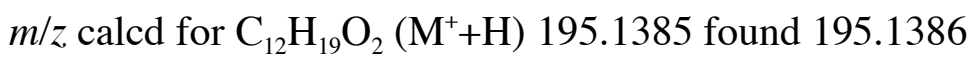

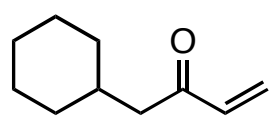

2

1-cyclohexylbut-3-en-2-one (2): FTIR 3094, 3020, 2931, 2852, 1700, 1682, $1615 \mathrm{~cm}^{-1}$; ${ }^{1} \mathrm{H}$ NMR (300 MHz in $\left.\mathrm{CDCl}_{3}\right) \delta$ 0.88-1.02 (2H, m), 1.07-1.35 (3H, m), 1.61-1.74 (5H, m), 1.78-1.93 (1H, m), $2.45(2 \mathrm{H}, \mathrm{d}, J=6.9 \mathrm{~Hz}), 5,81(1 \mathrm{H}, \mathrm{dd}, J=10.2,1.4 \mathrm{~Hz}), 6,20$ $(1 \mathrm{H}, \mathrm{dd}, J=17.6,1.4 \mathrm{~Hz}), 6.36(1 \mathrm{H}, \mathrm{dd}, J=17.6,10.2 \mathrm{~Hz}) ;{ }^{13} \mathrm{C}$ NMR $(75 \mathrm{MHz}$ in $\left.\mathrm{CDCl}_{3}\right) \delta 26.00 \mathrm{t}, 26.00 \mathrm{t}, 26.09 \mathrm{t}, 33.17 \mathrm{t}, 33.17 \mathrm{t}, 34.11 \mathrm{~d}, 47.15 \mathrm{t}, 127.74 \mathrm{t}, 136.89 \mathrm{~d}$, 200.58s; MS (CI) $m / z 153\left(\mathrm{M}^{+}+\mathrm{H}\right)$; HRMS (CI) $m / z$ calcd for $\mathrm{C}_{10} \mathrm{H}_{17} \mathrm{O}\left(\mathrm{M}^{+}+\mathrm{H}\right)$ 153.1279 found 153.1260 
<smiles>C=C(NC(=C)C(=O)CC1CCCCC1)C(=O)CC1CCCCC1</smiles>

3

bis (4-cyclohexyl-3-oxobut-1-en-2-yl) mercury (3): FTIR 3051, 2923, 2850, 1645 $\mathrm{cm}^{-1} ;{ }^{1} \mathrm{H}$ NMR $\left(300 \mathrm{MHz}\right.$ in $\mathrm{CDCl}_{3}$ ) $\delta$ 0.87-1.01 (4H, m), 1.06-1.35 (6H, m), 1.60-1.74 $(10 \mathrm{H}, \mathrm{m}), 1.75-1.90(2 \mathrm{H}, \mathrm{m}), 2.51(4 \mathrm{H}, \mathrm{d}, J=6.9 \mathrm{~Hz}), 6.06(2 \mathrm{H}, \mathrm{d}, J=1.2 \mathrm{~Hz}), 6.68$ $(2 \mathrm{H}, \mathrm{d}, J=1.2 \mathrm{~Hz}) ;{ }^{13} \mathrm{C} \mathrm{NMR}\left(75 \mathrm{MHz}\right.$ in $\left.\mathrm{CDCl}_{3}\right) \delta 26.14 \mathrm{t}, 26.14 \mathrm{t}, 26.14 \mathrm{t}, 26.14 \mathrm{t}$, $26.22 \mathrm{t}, 26.22 \mathrm{t}, 33.42 \mathrm{t}, 33.42 \mathrm{t}, 33.42 \mathrm{t}, 33.42 \mathrm{t}, 34.79 \mathrm{~d}, 34.79 \mathrm{~d}, 45.80 \mathrm{t}, 45.80 \mathrm{t}, 136.60 \mathrm{t}$, 136.60t, 180.04s, 180.04s, 207.21s, 207.21s; MS (CI) $m / z .505\left(\mathrm{M}^{+}+\mathrm{H}\right)$; HRMS (CI) $\mathrm{m} / \mathrm{z}$ calcd for $\mathrm{C}_{20} \mathrm{H}_{31} \mathrm{O}_{2} \mathrm{Hg}\left(\mathrm{M}^{+}+\mathrm{H}\right) 505.2030$ found 505.2020

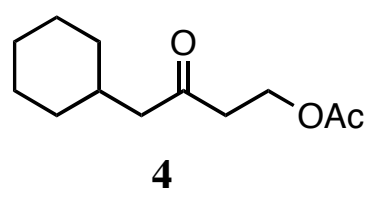

4-cyclohexyl-3-oxobutyl acetate (4): FTIR 2927, 2852, 1747, 1713, 1553, $1367 \mathrm{~cm}^{-1}$; ${ }^{1} \mathrm{H}$ NMR (300 MHz in $\left.\mathrm{CDCl}_{3}\right) \delta$ 0.86-1.00 (2H, m), 1.06-1.35 (3H, m), 1.60-1.73 (5H, m), 1.77-1.91 (1H, m), $2.02(3 \mathrm{H}, \mathrm{s}), 2.30(2 \mathrm{H}, \mathrm{d}, J=6.9 \mathrm{~Hz}), 2.71(2 \mathrm{H}, \mathrm{t}, J=6.3 \mathrm{~Hz})$, $4.32(2 \mathrm{H}, \mathrm{t}, J=6.3 \mathrm{~Hz}) ;{ }^{13} \mathrm{C} \mathrm{NMR}\left(75 \mathrm{MHz}\right.$ in $\left.\mathrm{CDCl}_{3}\right) \delta 20.76 \mathrm{q}, 25.98 \mathrm{t}, 25.98 \mathrm{t}, 26.08 \mathrm{t}$, 33.09t, 33.09t, 33.74d, 41.75t, 50.85t, 59.32t, 170.78s, 207.62s; MS (CI) m/z 213 $\left(\mathrm{M}^{+}+\mathrm{H}\right)$; HRMS (CI) $\mathrm{m} / z$, calcd for $\mathrm{C}_{12} \mathrm{H}_{21} \mathrm{O}_{3}\left(\mathrm{M}^{+}+\mathrm{H}\right) 213.1491$ found 213.1473<smiles>CC(=O)OCC(=O)CCC1CCCCC1</smiles>

5

4-cyclohexyl-2-oxobutyl acetate (5): FTIR 2925, 2851, 1751, 1733, $1374 \mathrm{~cm}^{-1} ;{ }^{1} \mathrm{H}$ NMR (300 MHz in $\left.\mathrm{CDCl}_{3}\right) \delta$ 0.81-0.95 $(2 \mathrm{H}, \mathrm{m}), 1.09-1.29(4 \mathrm{H}, \mathrm{m}), 1.50(2 \mathrm{H}, \mathrm{q}, J=7.7$ $\mathrm{Hz}), 1.60-1.74(5 \mathrm{H}, \mathrm{m}), 2.17(3 \mathrm{H}, \mathrm{s}), 2.42(2 \mathrm{H}, \mathrm{t}, J=7.7 \mathrm{~Hz}), 4.65(2 \mathrm{H}, \mathrm{s}) ;{ }^{13} \mathrm{C} \mathrm{NMR}$ $\left(75 \mathrm{MHz}\right.$ in $\left.\mathrm{CDCl}_{3}\right) \delta 20.25 \mathrm{q}, 26.01 \mathrm{t}, 26.01 \mathrm{t}, 26.31 \mathrm{t}, 30.39 \mathrm{t}, 32.85 \mathrm{t}, 32.85 \mathrm{t}, 36.15 \mathrm{t}$, 37.00d, 67.71t, 170.00s, 204.01s; MS (CI) $m / z 213\left(\mathrm{M}^{+}+\mathrm{H}\right)$; HRMS (CI) $m / z$ calcd for 


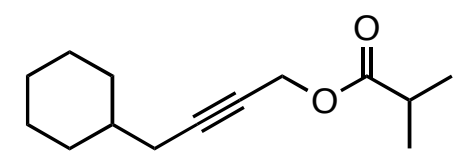

$6 a$

4-cyclohexylbut-2-ynyl isobutyrate (6a): FTIR 3464, 2934, 2857, 2306, 2237, 1748 $\mathrm{cm}^{-1} ;{ }^{1} \mathrm{H}\left(300 \mathrm{MHz}\right.$ in $\left.\mathrm{CDCl}_{3}\right) \delta 0.90-1.05(2 \mathrm{H}, \mathrm{m}), 1.18(6 \mathrm{H}, \mathrm{d}, J=7.0 \mathrm{~Hz}), 1.10-1.31$ $(3 \mathrm{H}, \mathrm{m}), 1.38-1.54(1 \mathrm{H}, \mathrm{m}), 1.61-1.83(5 \mathrm{H}, \mathrm{m}), 2.11(2 \mathrm{H}, \mathrm{dt}, J=6.7,2.2 \mathrm{~Hz}), 2.59(1 \mathrm{H}$, septet, $J=7.0 \mathrm{~Hz}), 4.67(2 \mathrm{H}, \mathrm{t}, J=2.2 \mathrm{~Hz}) ;{ }^{13} \mathrm{C} \mathrm{NMR}\left(75 \mathrm{MHz}\right.$ in $\left.\mathrm{CDCl}_{3}\right) \delta 18.73 \mathrm{q}$, $18.73 \mathrm{q}, 25.96 \mathrm{t}, 25.96 \mathrm{t}, 26.11 \mathrm{t}, 26.40 \mathrm{t}, 3250 \mathrm{t}, 32.50 \mathrm{t}, 33.70 \mathrm{~d}, 37.03 \mathrm{~d}, 52.54 \mathrm{t}, 74.93 \mathrm{~s}$, 86.07s, 176.19s; MS (CI) m/z, $223\left(\mathrm{M}^{+}+\mathrm{H}\right)$; HRMS (CI) $m / z$ calcd for $\mathrm{C}_{14} \mathrm{H}_{23} \mathrm{O}_{2}\left(\mathrm{M}^{+}+\mathrm{H}\right)$ 223.1698 found 223.1679

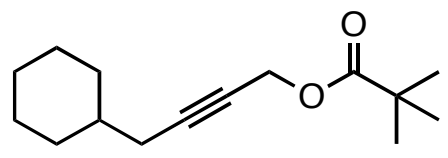

6b

4-cyclohexylbut-2-ynyl pivalate (6b): FTIR 3450, 2939, 2853, 2305, 2236, 1746, 1365 $\mathrm{cm}^{-1} ;{ }^{1} \mathrm{H}$ NMR (300 MHz in $\left.\mathrm{CDCl}_{3}\right) \delta$ 0.91-1.05 (2H, m), 1.09-1.31 $(3 \mathrm{H}, \mathrm{m}), 1.22(9 \mathrm{H}$, s), 1.39-1.53 (1H, m), 1.61-1.82 (5H, m), $2.10(2 \mathrm{H}, \mathrm{dt}, J=6.6,2.3 \mathrm{~Hz}), 4.65(2 \mathrm{H}, \mathrm{t}, J=$ $2.3 \mathrm{~Hz}$ ); ${ }^{13} \mathrm{C} \mathrm{NMR}\left(75 \mathrm{MHz}\right.$ in $\left.\mathrm{CDCl}_{3}\right) \delta 25.99 \mathrm{t}, 25.99 \mathrm{t}, 26.13 \mathrm{t}, 26.41 \mathrm{t}, 26.96 \mathrm{q}, 26.96 \mathrm{q}$, 26.96q, 32.50t, 32.50t, 37.07d, 38.57s, 52.65t, 75.11s, 85.88s, 177.59s; MS (CI) $\mathrm{m} / \mathrm{z}$ $237\left(\mathrm{M}^{+}+\mathrm{H}\right)$; HRMS (CI) $m / z$ calcd for $\mathrm{C}_{15} \mathrm{H}_{25} \mathrm{O}_{2}\left(\mathrm{M}^{+}+\mathrm{H}\right) 237.1854$ found 237.1854

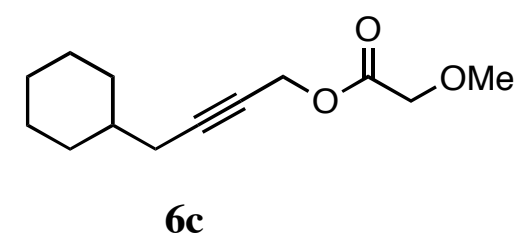

4-cyclohexylbut-2-ynyl 2-methoxyacetate (6c): FTIR 2938, 2853, 2307, 2237, 1769 $\mathrm{cm}^{-1} ;{ }^{1} \mathrm{H}$ NMR (300 MHz in $\mathrm{CDCl}_{3}$ ) $\delta$ 0.90-1.04 (2H, m), 1.05-1.31 (3H, m), 1.39-1.53 $(1 \mathrm{H}, \mathrm{m}), 1.61-1.82(5 \mathrm{H}, \mathrm{m}), 2.11(2 \mathrm{H}, \mathrm{dt}, J=6.7,2.2 \mathrm{~Hz}), 3.46(3 \mathrm{H}, \mathrm{s}), 4.07(2 \mathrm{H}, \mathrm{s})$, $4.77(2 \mathrm{H}, \mathrm{t}, J=2.2 \mathrm{~Hz}) ;{ }^{13} \mathrm{C}$ NMR $\left(75 \mathrm{MHz}\right.$ in $\left.\mathrm{CDCl}_{3}\right) \delta 25.87 \mathrm{t}, 25.87 \mathrm{t}, 26.01 \mathrm{t}, 26.31 \mathrm{t}$, 
32.44t, 32.44t, 36.89d, 52.92t, 59.11q, 69.45t, 74.17s, 86.80s, 169.41s; MS (CI) m/z 225 $\left(\mathrm{M}^{+}+\mathrm{H}\right)$; HRMS (CI) $m / z$ calcd for $\mathrm{C}_{13} \mathrm{H}_{21} \mathrm{O}_{3}\left(\mathrm{M}^{+}+\mathrm{H}\right) 225.1491$ found 225.1493

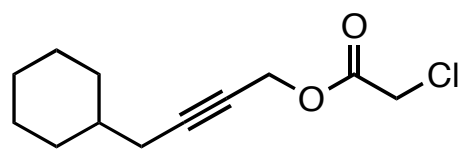

6d

4-cyclohexylbut-2-ynyl 2-chloroacetate (6d): FTIR 2929, 2852, 2309, 2237, 1768, $975 \mathrm{~cm}^{-1} ;{ }^{1} \mathrm{H}$ NMR (300 MHz in $\left.\mathrm{CDCl}_{3}\right) \delta$ 0.90-1.05 (2H, m), 1.06-1.32 (3H, m), 1.40-1.53 (1H, m), 1.61-1.82 (5H, m), $2.12(2 \mathrm{H}, \mathrm{dt}, J=6.7,2.2 \mathrm{~Hz}), 4.10(2 \mathrm{H}, \mathrm{s}), 4.79$ $(2 \mathrm{H}, \mathrm{t}, J=2.2 \mathrm{~Hz}) ;{ }^{13} \mathrm{C} \mathrm{NMR}\left(75 \mathrm{MHz}\right.$ in $\left.\mathrm{CDCl}_{3}\right) \delta 25.95 \mathrm{t}, 25.95 \mathrm{t}, 26.09 \mathrm{t}, 26.40 \mathrm{t}, 32.53 \mathrm{t}$, 32.53t, 36.95d, 40.59t, 54.37t, 73.80s, 87.49s, 166.59s; MS (CI) m/z $229\left(\mathrm{M}^{+}+\mathrm{H}\right)$; HRMS $m / z$ calcd for $\mathrm{C}_{12} \mathrm{H}_{18} \mathrm{O}_{2} \mathrm{Cl}\left(\mathrm{M}^{+}+\mathrm{H}\right) 229.0996$ found 229.1008

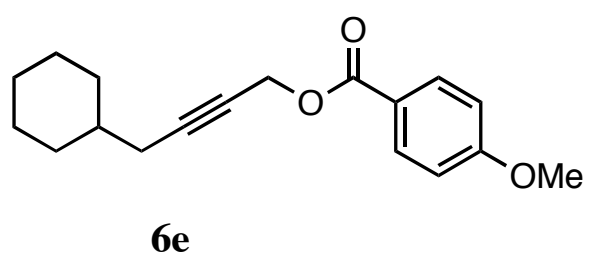

4-cyclohexylbut-2-ynyl 4-methoxybenzoate (6e): FTIR 2926, 2851, 2308, 2237, 2050, 1917, 1724, 1607, $1511 \mathrm{~cm}^{-1}$; ${ }^{1} \mathrm{H}$ NMR (300 MHz in $\left.\mathrm{CDCl}_{3}\right) \delta$ 0.91-1.06 (2H, m), 1.06-1.32 (3H, m), 1.41-1.54 (1H, m), 1.60-1.85 (5H, m), $2.13(2 \mathrm{H}, \mathrm{dt}, J=6.7,2.2 \mathrm{~Hz})$, $3.86(3 \mathrm{H}, \mathrm{s}), 4.89(2 \mathrm{H}, \mathrm{t}, J=2.2 \mathrm{~Hz}), 6.92(2 \mathrm{H}, \mathrm{d}, J=9.1 \mathrm{~Hz}), 8.03(2 \mathrm{H}, \mathrm{d}, J=9.1 \mathrm{~Hz})$; ${ }^{13} \mathrm{C}$ NMR $\left(75 \mathrm{MHz}\right.$ in $\left.\mathrm{CDCl}_{3}\right) \delta 25.94 \mathrm{t}, 25.94 \mathrm{t}, 26.07 \mathrm{t}, 26.42 \mathrm{t}, 32.50 \mathrm{t}, 32.50 \mathrm{t}, 36.98 \mathrm{~d}$, 52.86t, 55.16q, 75.02s, 86.23s, 113.41d, 113.41d, 122.04s, 131.61d, 131.61d, 163.34s, $165.47 \mathrm{~s} ; \mathrm{MS}(\mathrm{CI}) \mathrm{m} / z 287\left(\mathrm{M}^{+}+\mathrm{H}\right)$; HRMS (CI) $m / z$ calcd for $\mathrm{C}_{18} \mathrm{H}_{23} \mathrm{O}_{3}\left(\mathrm{M}^{+}+\mathrm{H}\right)$ 287.1647 , found 287.1640<smiles>O=C(OCC#CCC1CCCCC1)c1ccc([N+](=O)[O-])cc1</smiles>

6f

4-cyclohexylbut-2-ynyl 4-nitrobenzoate (6f): FTIR 3442, 3112, 3083, 3056, 2926, 2852, 2310, 2237, 1949, 1806, 1737, 1608, 1535, $719 \mathrm{~cm}^{-1} ;{ }^{1} \mathrm{H}$ NMR $(200 \mathrm{MHz}$ in 
$\left.\mathrm{CDCl}_{3}\right) \delta$ 0.88-1.11 (2H, m), 1.13-1.35 (3H, m), 1.40-1.53 (1H, m), 1.60-1.86 (5H, m), $2.14(2 \mathrm{H}, \mathrm{dt}, J=6.6,2.2 \mathrm{~Hz}), 4.97(2 \mathrm{H}, \mathrm{t}, J=2.2 \mathrm{~Hz}), 8.22-8.34(4 \mathrm{H}, \mathrm{m}) ;{ }^{13} \mathrm{C} \mathrm{NMR}(50$ $\mathrm{MHz}$ in $\left.\mathrm{CDCl}_{3}\right) \delta 25.94 \mathrm{t}, 25.94 \mathrm{t}, 26.08 \mathrm{t}, 26.42 \mathrm{t}, 32.55 \mathrm{t}, 32.55 \mathrm{t}, 36.99 \mathrm{~d}, 54.17 \mathrm{t}, 74.19 \mathrm{~s}$, 87.44s, 123.51d, 123.51d, 130.88d, 130.88d, 135.22s, 150.71s, 164.14s; MS (CI) m/z $302\left(\mathrm{M}^{+}+\mathrm{H}\right)$; HRMS (CI) $\mathrm{m} / z$ calcd for $\mathrm{C}_{17} \mathrm{H}_{20} \mathrm{NO}_{4}\left(\mathrm{M}^{+}+\mathrm{H}\right) 302.1393$ found 302.1382

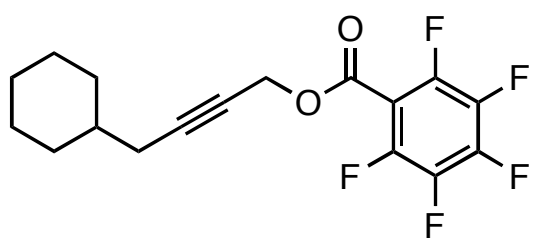

$6 \mathrm{~g}$

4-cyclohexylbut-2-ynyl 2, 3, 4, 5, 6-pentafluorobenzoate (6g): FTIR 3479, 2927, 2853, 2311, 2239, 1748, 1652, 1524, $1506 \mathrm{~cm}^{-1} ;{ }^{1} \mathrm{H}$ NMR $\left(300 \mathrm{MHz}\right.$ in $\left.\mathrm{CDCl}_{3}\right) \delta$ 0.92-1.06 (2H, m), 1.06-1.33 (3H, m), 1.41-1.55 (1H, m), 1.60-1.83 (5H, m), $2.13(2 \mathrm{H}$, dt, $J=6.6,2.2 \mathrm{~Hz}), 4.96(2 \mathrm{H}, \mathrm{t}, J=2.2 \mathrm{~Hz}) ;{ }^{13} \mathrm{C} \mathrm{NMR}\left(75 \mathrm{MHz}\right.$ in $\left.\mathrm{CDCl}_{3}\right) \delta 26.06 \mathrm{t}$, $26.06 \mathrm{t}, 26.18 \mathrm{t}, 26.49 \mathrm{t}, 32.61 \mathrm{t}, 32.61 \mathrm{t}, 37.09 \mathrm{~d}, 54.97 \mathrm{t}, 73.53 \mathrm{~s}, 88.03 \mathrm{~s}, 137.70\left(\mathrm{~d}, J_{\mathrm{CF}}=\right.$ $257.1 \mathrm{~Hz}), 143.33\left(\mathrm{~d}, J_{\mathrm{CF}}=260.0 \mathrm{~Hz}\right), 145.47\left(\mathrm{~d}, J_{\mathrm{CF}}=258.5 \mathrm{~Hz}\right), 158.46 \mathrm{~s}$; MS (CI) $\mathrm{m} / \mathrm{z}$ $347\left(\mathrm{M}^{+}+\mathrm{H}\right)$; HRMS (CI) $m / z$ calcd for $\mathrm{C}_{17} \mathrm{H}_{16} \mathrm{O}_{2} \mathrm{~F}_{5}\left(\mathrm{M}^{+}+\mathrm{H}\right) 347.1070$ found 347.1066

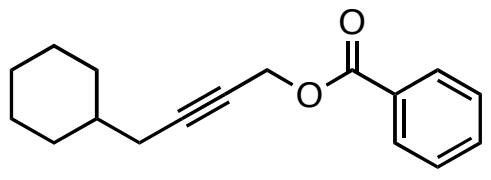

6h

4-cyclohexylbut-2-ynyl benzoate (6h): FTIR 3431, 3087, 3063, 3033, 2934, 2852, 2308, 2237, 2056, 1965, 1909, 1819, 1732, $1602 \mathrm{~cm}^{-1} ;{ }^{1} \mathrm{H}$ NMR $\left(300 \mathrm{MHz}\right.$ in $\left.\mathrm{CDCl}_{3}\right) \delta$ 0.92-1.06 (2H, m), 1.09-1.32 (3H, m), 1.41-1.55 (1H, m), 1.60-1.85 (5H, m), $2.13(2 \mathrm{H}$, $\mathrm{dt}, J=6.6,2.2 \mathrm{~Hz}), 4.93(2 \mathrm{H}, \mathrm{t}, J=2.2 \mathrm{~Hz}), 7.42-7.48(2 \mathrm{H}, \mathrm{m}), 7.54-7.61(1 \mathrm{H}, \mathrm{m})$, 8.05-8.10 (2H, m); ${ }^{13} \mathrm{C}$ NMR (75 MHz in $\mathrm{CDCl}_{3}$ ) $\delta 26.00 t, 26.00 t, 26.14 \mathrm{t}, 26.49 \mathrm{t}, 32.57 \mathrm{t}$, $32.57 \mathrm{t}, 37.04 \mathrm{~d}, 53.26 \mathrm{t}, 74.84 \mathrm{~s}, 86.55 \mathrm{~s}, 128.23 \mathrm{~d}, 128.23 \mathrm{~d}, 129.65 \mathrm{~d}, 129.65 \mathrm{~d}, 129.75 \mathrm{~s}$, 132.96d, 165.84s; MS (CI) $\mathrm{m} / z 257\left(\mathrm{M}^{+}+\mathrm{H}\right)$; HRMS (CI) $\mathrm{m} / \mathrm{z}$ calcd for $\mathrm{C}_{17} \mathrm{H}_{21} \mathrm{O}_{2}$ $\left(\mathrm{M}^{+}+\mathrm{H}\right) 257.1542$ found 257.1537 


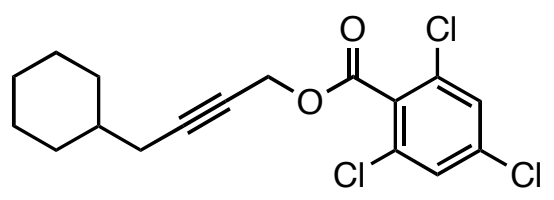

6i

4-cyclohexylbut-2-ynyl 2, 4, 6-trichlorobenzoate (6i): FTIR 3478, 3152, 3129, 3081, 2933, 2852, 2310, 2238, 1958, 1757, 1582, $1551 \mathrm{~cm}^{-1}$; ${ }^{1} \mathrm{H}$ NMR $\left(300 \mathrm{MHz}\right.$ in $\left.\mathrm{CDCl}_{3}\right) \delta$ 0.92-1.06 (2H, m), 1.06-1.31 (3H, m), 1.41-1.50 (1H, m), 1.60-1.84 (5H, m), $2.13(2 \mathrm{H}$, $\mathrm{dt}, J=6.7,2.3 \mathrm{~Hz}), 4.96(2 \mathrm{H}, \mathrm{t}, J=2.3 \mathrm{~Hz}), 7.35(2 \mathrm{H}, \mathrm{s}) ;{ }^{13} \mathrm{C} \mathrm{NMR}\left(75 \mathrm{MHz}\right.$ in $\left.\mathrm{CDCl}_{3}\right)$ ठ 26.02t, 26.02t, 26.15t, 26.49t, 32.57t, 32.57t, 37.03d, 54.65t, 73.72s, 87.68s, 127.94d, $127.94 \mathrm{~d}, 131.58 \mathrm{~s}, 132.78 \mathrm{~s}, 132.78 \mathrm{~s}, 136.25 \mathrm{~s}, 163.31 \mathrm{~s} ; \mathrm{MS}(\mathrm{CI}) \mathrm{m} / \mathrm{z} 359\left(\mathrm{M}^{+}+\mathrm{H}\right)$; HRMS (CI) $m / z$ calcd for $\mathrm{C}_{17} \mathrm{H}_{18} \mathrm{O}_{2} \mathrm{Cl}_{3}\left(\mathrm{M}^{+}\right.$

$+\mathrm{H}) 359.0373$ found 359.0373

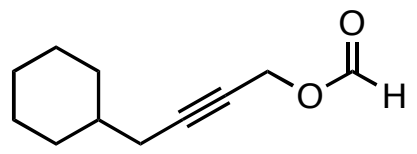

6j

4-cyclohexylbut-2-ynyl formate (6j): FTIR 3441, 2928, 2852, 2310, 2238, $1738 \mathrm{~cm}^{-1}$; ${ }^{1} \mathrm{H}$ NMR $\left(300 \mathrm{MHz}\right.$ in $\left.\mathrm{CDCl}_{3}\right) \delta$ 0.91-1.05 (2H, m), 1.06-1.32 $(3 \mathrm{H}, \mathrm{m}), 1.40-1.54(1 \mathrm{H}$, m), 1.62-1.83 (5H, m), $2.12(2 \mathrm{H}, \mathrm{dt}, J=6.9,2.2 \mathrm{~Hz}), 4.77(2 \mathrm{H}, \mathrm{dt}, J=2.2,0.9 \mathrm{~Hz}), 8.07$ $(1 \mathrm{H}, \mathrm{t}, J=0.9 \mathrm{~Hz}) ;{ }^{13} \mathrm{C} \mathrm{NMR}\left(75 \mathrm{MHz}\right.$ in $\left.\mathrm{CDCl}_{3}\right) \delta 25.84 \mathrm{t}, 25.84 \mathrm{t}, 25.97 \mathrm{t}, 26.23 \mathrm{t}, 32.39 \mathrm{t}$, 32.39t, 36.85d, 51.94t, 73.99s, 86.71s, 159.88d; MS (CI) m/z $181\left(\mathrm{M}^{+}+\mathrm{H}\right)$; HRMS (CI) $m / z$ calcd for $\mathrm{C}_{11} \mathrm{H}_{17} \mathrm{O}_{2}\left(\mathrm{M}^{+}+\mathrm{H}\right) 181.1229$ found 181.1222

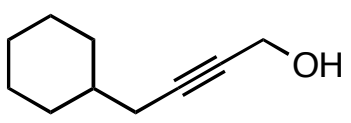

6k

4-cyclohexylbut-2-yn-1-ol (6k): FTIR 3343, 2935, 2854, 2287, 2224, $\mathrm{cm}^{-1}$; ${ }^{1} \mathrm{H}$ NMR (300 MHz in $\left.\mathrm{CDCl}_{3}\right) \delta$ 0.91-1.05 (2H, m) , 1.06-1.32 (3H, m) , 1.38-1.53 (1H, m) , 1.48 $(1 \mathrm{H}, \mathrm{t}, J=6.1 \mathrm{~Hz}), 1.61-1.84(5 \mathrm{H}, \mathrm{m}), 2.11(2 \mathrm{H}, \mathrm{dt}, J=6.6,2.2 \mathrm{~Hz}), 4.26(2 \mathrm{H}, \mathrm{dt}, J=$ 6.1, $2.2 \mathrm{~Hz}) ;{ }^{13} \mathrm{C}$ NMR $\left(75 \mathrm{MHz}\right.$ in $\left.\mathrm{CDCl}_{3}\right) \delta 25.93 \mathrm{t}, 25.93 \mathrm{t}, 26.08 \mathrm{t}, 26.37 \mathrm{t}, 32.51 \mathrm{t}$, 32.51t, 37.09d, 50.90t, 79.15s, 84.86s; MS (CI) m/z $135\left(\mathrm{M}^{+}-\mathrm{OH}\right), m / z 151\left(\mathrm{M}^{+}-\mathrm{H}\right)$; HRMS (CI) $m / z$ calcd for $\mathrm{C}_{10} \mathrm{H}_{15}\left(\mathrm{M}^{+}-\mathrm{OH}\right) 135.1173$ found $135.1144, \mathrm{~m} / \mathrm{z}$ calcd for 


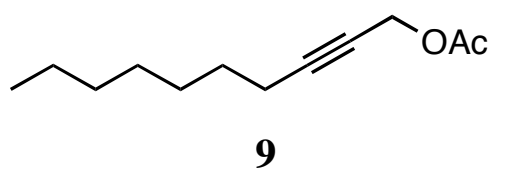

dec-2-ynyl acetate (9): FTIR 2930, 2857, 2308, 2237, 1749, $1378 \mathrm{~cm}^{-1}$; ${ }^{1} \mathrm{H}$ NMR (300 $\mathrm{MHz}$ in $\left.\mathrm{CDCl}_{3}\right) \delta 0.88(3 \mathrm{H}, \mathrm{t}, J=6.6 \mathrm{~Hz}), 1.24-1.40(8 \mathrm{H}, \mathrm{m}), 1.46-1.56(2 \mathrm{H}, \mathrm{m}), 2.09$ $(3 \mathrm{H}, \mathrm{s}), 2.22(2 \mathrm{H}, \mathrm{tt}, J=7.1,2.2 \mathrm{~Hz}), 4.66(2 \mathrm{H}, \mathrm{t}, J=2.2 \mathrm{~Hz}) ;{ }^{13} \mathrm{C} \mathrm{NMR}(75 \mathrm{MHz}$ in $\left.\mathrm{CDCl}_{3}\right) \delta 13.93 \mathrm{q}, 18.65 \mathrm{t}, 20.64 \mathrm{q}, 22.51 \mathrm{t}, 28.33 \mathrm{t}, 28.67 \mathrm{t}, 28.70 \mathrm{t}, 31.61 \mathrm{t}, 52.73 \mathrm{t}, 73.81 \mathrm{~s}$, 87.55s, 170.17s; MS (CI) $m / z, 197\left(\mathrm{M}^{+}+\mathrm{H}\right)$; HRMS (CI) $m / z$ calcd for $\mathrm{C}_{12} \mathrm{H}_{21} \mathrm{O}_{2}\left(\mathrm{M}^{+}+\mathrm{H}\right)$ 197.1542 found 197.1548

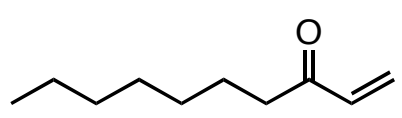

10

dec-1-en-3-one (10): FTIR 3095, 3023, 2958, 2928, 2856, 1705, 1683, $1615 \mathrm{~cm}^{-1}$; ${ }^{1} \mathrm{H}$ NMR $\left(300 \mathrm{MHz}\right.$ in $\left.\mathrm{CDCl}_{3}\right) \delta 0.88(3 \mathrm{H}, \mathrm{t}, J=6.8 \mathrm{~Hz}), 1.24-1.36(8 \mathrm{H}, \mathrm{m}), 1.62(2 \mathrm{H}$, quintet, $J=7.4 \mathrm{~Hz}), 2.57(2 \mathrm{H}, \mathrm{t}, J=7.4 \mathrm{~Hz}), 5.81(1 \mathrm{H}, \mathrm{dd}, J=10.3,1.4 \mathrm{~Hz}), 6.21(1 \mathrm{H}$, $\mathrm{dd}, J=17.7,1.4 \mathrm{~Hz}), 6.36(1 \mathrm{H}, \mathrm{dd}, J=17.7,10.3 \mathrm{~Hz}) ;{ }^{13} \mathrm{C} \mathrm{NMR}\left(75 \mathrm{MHz}\right.$ in $\left.\mathrm{CDCl}_{3}\right) \delta$ 13.92q, 22.49t, 23.91t, 28.98t, 29.11t, 31.58t, 39.54t, 127.61t, 136.51d, 200.84s; MS (CI) $m / z 155\left(\mathrm{M}^{+}+\mathrm{H}\right)$; HRMS (CI) $m / z$ calcd for $\mathrm{C}_{10} \mathrm{H}_{19} \mathrm{O}\left(\mathrm{M}^{+}+\mathrm{H}\right) 155.1436$ found 155.1410

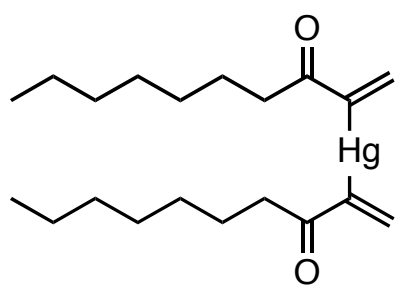

11

bis (3-oxodec-1-en-2-yl) mercury (11): ${ }^{1} \mathrm{H}$ NMR $\left(200 \mathrm{MHz}\right.$ in $\left.\mathrm{CDCl}_{3}\right) \delta 0.88(6 \mathrm{H}, \mathrm{t}, J=$ $6.5 \mathrm{~Hz}), 1.22-1.36(16 \mathrm{H}, \mathrm{m}), 1.52-1.68(4 \mathrm{H}, \mathrm{m}), 2.64(4 \mathrm{H}, \mathrm{t}, J=7.6 \mathrm{~Hz}), 6.07(2 \mathrm{H}, \mathrm{d}, J=$ $1.1 \mathrm{~Hz}), 6.69(2 \mathrm{H}, \mathrm{d}, J=1.1 \mathrm{~Hz}) ; \mathrm{MS}(\mathrm{CI}) m / z .509\left(\mathrm{M}^{+}+\mathrm{H}\right)$ 


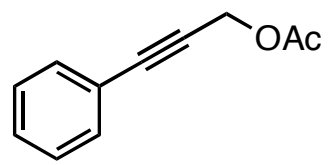

12

3-phenylprop-2-ynyl acetate (12): FTIR 3472, 3078, 3057, 3036, 3023, 2940, 2237, 2136, 2046, 1955, 1885, 1743, 758, $691 \mathrm{~cm}^{-1} ;{ }^{1} \mathrm{H}$ NMR $\left(200 \mathrm{MHz}\right.$ in $\left.\mathrm{CDCl}_{3}\right) \delta 2.14(3 \mathrm{H}$, s), $4.91(2 \mathrm{H}, \mathrm{s}), 7.28-7.36(3 \mathrm{H}, \mathrm{m}), 7.43-7.50(2 \mathrm{H}, \mathrm{m}) ;{ }^{13} \mathrm{C} \mathrm{NMR}\left(50 \mathrm{MHz}\right.$ in $\left.\mathrm{CDCl}_{3}\right) \delta$ 20.33q, 52.41t, 82.88s, 86.10s, 121.91s, 128.04d, 128.04d, 128.47d, 131.58d, 131.58d, 169.79s; MS (CI) $m / z, 174\left(\mathrm{M}^{+}\right)$; HRMS (CI) $m / z$ calcd for $\mathrm{C}_{11} \mathrm{H}_{10} \mathrm{O}_{2}\left(\mathrm{M}^{+}\right) 174.0680$ found 174.0693<smiles>C=CC(=O)c1ccccc1</smiles>

13

1-phenylprop-2-en-1-one (13): FTIR 3087, 3062, 3025, 2976, 2930, 1965, 1818, 1674, $1608,1597,1578,730,689 \mathrm{~cm}^{-1} ;{ }^{1} \mathrm{H}$ NMR $\left(300 \mathrm{MHz}\right.$ in $\left.\mathrm{CDCl}_{3}\right) \delta 5.94(1 \mathrm{H}, \mathrm{dd}, J=$ $10.4,1.7 \mathrm{~Hz}), 6.44(1 \mathrm{H}, \mathrm{dd}, J=17.2,1.7 \mathrm{~Hz}), 7.17(1 \mathrm{H}, \mathrm{dd}, J=17.2,10.4 \mathrm{~Hz})$, 7.46-7.52 (2H, m), 7.56-7.62 (1H, m), 7.93-7.98 (2H, m); ${ }^{13} \mathrm{C} \mathrm{NMR}\left(75 \mathrm{MHz}\right.$ in $\left.\mathrm{CDCl}_{3}\right)$ $\delta 128.55 \mathrm{~d}, 128.55 \mathrm{~d}, 128.61 \mathrm{~d}, 128.61 \mathrm{~d}, 130.05 \mathrm{t}, 132.32 \mathrm{~d}, 132.91 \mathrm{~d}, 137.20 \mathrm{~s}, 190.93 \mathrm{~s}$; MS (CI) $m / z 133\left(\mathrm{M}^{+}+\mathrm{H}\right)$; HRMS (CI) $m / z$ calcd for $\mathrm{C}_{9} \mathrm{H}_{9} \mathrm{O}\left(\mathrm{M}^{+}+\mathrm{H}\right) 133.0653$ found 133.0646

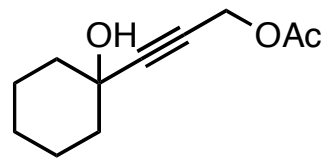

14

3-(1-hydroxycyclohexyl) prop-2-ynyl acetate (14): FTIR 3437, 2938, 2859, 2237, $1748 \mathrm{~cm}^{-1} ;{ }^{1} \mathrm{H}$ NMR (300 MHz in $\left.\mathrm{CDCl}_{3}\right) \delta$ 1.21-1.32 (1H, m), 1.46-1.63 (4H, m), 1.64-1.73 (3H, m), 1.86-1.95 (2H, m), 1.95-2.01 (1H, m), $2.10(3 \mathrm{H}, \mathrm{s}), 4.72(2 \mathrm{H}, \mathrm{s}) ;{ }^{13} \mathrm{C}$ NMR $\left(75 \mathrm{MHz}\right.$ in $\left.\mathrm{CDCl}_{3}\right) \delta 20.49 \mathrm{q}, 22.87 \mathrm{t}, 22.87 \mathrm{t}, 24.89 \mathrm{t}, 39.36 \mathrm{t}, 39.36 \mathrm{t}, 52.26 \mathrm{t}$, 68.07s, 77.50s, 90.42s, 170.22s; MS (CI) m/z $179\left(\mathrm{M}^{+}-\mathrm{OH}\right)$; HRMS (CI) $m / z$ calcd for $\mathrm{C}_{11} \mathrm{H}_{15} \mathrm{O}_{2}\left(\mathrm{M}^{+}-\mathrm{OH}\right) 179.1072$ found 179.1063 


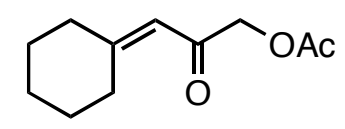

15

3-cyclohexylidene-2-oxopropyl acetate (15): FTIR 2931, 2856, 1754, 1705, 1627 $\mathrm{cm}^{-1} ;{ }^{1} \mathrm{H}$ NMR $\left(300 \mathrm{MHz}\right.$ in $\left.\mathrm{CDCl}_{3}\right) \delta 1.58-1.73(6 \mathrm{H}, \mathrm{m}), 2.18(3 \mathrm{H}, \mathrm{s}), 2.21(2 \mathrm{H}, \mathrm{t}, J=$ $5.2 \mathrm{~Hz}), 2.84(2 \mathrm{H}, \mathrm{t}, J=5.6 \mathrm{~Hz}), 4.67(2 \mathrm{H}, \mathrm{s}), 5.94(1 \mathrm{H}, \mathrm{s}) ;{ }^{13} \mathrm{C} \mathrm{NMR}(75 \mathrm{MHz}$ in $\left.\mathrm{CDCl}_{3}\right) \delta 20.47 \mathrm{q}, 26.00 \mathrm{t}, 27.80 \mathrm{t}, 28.68 \mathrm{t}, 30.38 \mathrm{t}, 38.22 \mathrm{t}, 68.75 \mathrm{t}, 115.93 \mathrm{~d}, 166.03 \mathrm{~s}$, 170.16s, 193.14s; MS (CI) m/z $197\left(\mathrm{M}^{+}+\mathrm{H}\right)$; HRMS (CI) $\mathrm{m} / \mathrm{z}$ calcd for $\mathrm{C}_{11} \mathrm{H}_{17} \mathrm{O}_{3}$ $\left(\mathrm{M}^{+}+\mathrm{H}\right)$ 197.1177 found 197.1157

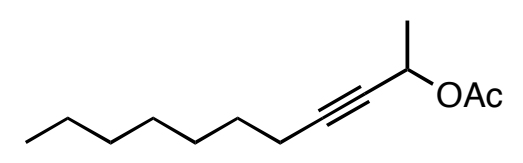

16

undec-3-yn-2-yl acetate (16): FTIR 3469, 2988, 2935, 2858, 2248, 1750, $1371 \mathrm{~cm}^{-1}$; ${ }^{1} \mathrm{H}$ NMR $\left(300 \mathrm{MHz}\right.$ in $\left.\mathrm{CDCl}_{3}\right) \delta 0.88(3 \mathrm{H}, \mathrm{t}, J=6.7 \mathrm{~Hz}), 1.25-1.38(8 \mathrm{H}, \mathrm{m}), 1.46(3 \mathrm{H}, \mathrm{d}, J$ $=6.7 \mathrm{~Hz}), 1.44-1.53(2 \mathrm{H}, \mathrm{m}), 2.06(3 \mathrm{H}, \mathrm{s}), 2.19(2 \mathrm{H}, \mathrm{dt}, J=7.2,2.0 \mathrm{~Hz}), 5.44(1 \mathrm{H}, \mathrm{tq}, J$ $=6.7,2.0 \mathrm{~Hz}) ;{ }^{13} \mathrm{C} \mathrm{NMR}\left(75 \mathrm{MHz}\right.$ in $\left.\mathrm{CDCl}_{3}\right) \delta 13.91 \mathrm{q}, 18.52 \mathrm{t}, 20.95 \mathrm{q}, 21.67 \mathrm{q}, 22.47 \mathrm{t}$, 28.36t, 28.64t, 28.64t, 31.59t, 60.68d, 78.50s, 85.39s, 169.73s; MS (CI) m/z 211 $\left(\mathrm{M}^{+}+\mathrm{H}\right)$; HRMS (CI) $m / z$ calcd for $\mathrm{C}_{13} \mathrm{H}_{23} \mathrm{O}_{2}\left(\mathrm{M}^{+}+\mathrm{H}\right) 211.1698$ found 211.1720

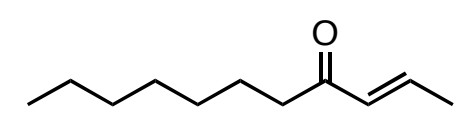

17

(E)-undec-2-en-4-one (17): FTIR 3036, 2958, 2929, 2856, 1698, 1675, $1633 \mathrm{~cm}^{-1} ;{ }^{1} \mathrm{H}$ NMR $\left(600 \mathrm{MHz}\right.$ in $\left.\mathrm{CDCl}_{3}\right) \delta 0.88(3 \mathrm{H}, \mathrm{t}, J=7.1 \mathrm{~Hz}), 1.24-1.33(8 \mathrm{H}, \mathrm{m}), 1.60(2 \mathrm{H}$, quintet, $J=7.4 \mathrm{~Hz}), 1.90(3 \mathrm{H}, \mathrm{dd}, J=6.9,1.6 \mathrm{~Hz}), 2.51(2 \mathrm{H}, \mathrm{t}, J=7.4 \mathrm{~Hz}), 6.12(1 \mathrm{H}$, $\mathrm{dq}, J=15.9,1.6 \mathrm{~Hz}), 6.84(1 \mathrm{H}, \mathrm{dq}, J=15.9,6.9 \mathrm{~Hz}) ;{ }^{13} \mathrm{C} \mathrm{NMR}\left(75 \mathrm{MHz}\right.$ in $\left.\mathrm{CDCl}_{3}\right) \delta$ $13.89 \mathrm{q}, 18.01 \mathrm{q}, 22.47 \mathrm{t}, 24.17 \mathrm{t}, 28.97 \mathrm{t}, 29.15 \mathrm{t}, 31.56 \mathrm{t}, 39.89 \mathrm{t}, 131.85 \mathrm{~d}, 142.01 \mathrm{~d}$, 200.44s; MS (CI) $m / z 169\left(\mathrm{M}^{+}+\mathrm{H}\right)$; HRMS (CI) $m / z$ calcd for $\mathrm{C}_{11} \mathrm{H}_{21} \mathrm{O}\left(\mathrm{M}^{+}+\mathrm{H}\right)$ 169.1592 found 169.1588 


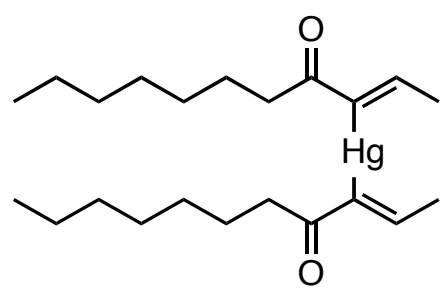

18

bis ((Z)-4-oxoundec-2-en-3-yl) mercury (18): FTIR 2953, 2926, 2854, 1638, 1612 $\mathrm{cm}^{-1} ;{ }^{1} \mathrm{H}\left(600 \mathrm{MHz}\right.$ in $\left.\mathrm{CDCl}_{3}\right) \delta 0.88(6 \mathrm{H}, \mathrm{t}, J=7.1 \mathrm{~Hz}), 1.23-1.33(16 \mathrm{H}, \mathrm{m}), 1.57-1.63$ $(4 \mathrm{H}, \mathrm{m}), 2.10(6 \mathrm{H}, \mathrm{d}, J=6.6 \mathrm{~Hz}), 2.61(4 \mathrm{H}, \mathrm{t}, J=7.6 \mathrm{~Hz}), 7.89(2 \mathrm{H}, \mathrm{q}, J=6.6 \mathrm{~Hz}) ;{ }^{13} \mathrm{C}$ NMR (75 MHz in $\left.\mathrm{CDCl}_{3}\right) \delta 14.10 \mathrm{q}, 14.10 \mathrm{q}, 20.35 \mathrm{q}, 20.35 \mathrm{q}, 22.65 \mathrm{t}, 22.65 \mathrm{t}, 25.08 \mathrm{t}$, 25.08t, 29.17t, 29.17t, 29.47t, 29.47t, 31.74t, 31.74t, 38.71t, 38.71t, 148.40d, 148.40d, 173.52s, 173.52s, 207.45s, 207.45s; MS (CI) m/z $537\left(\mathrm{M}^{+}+\mathrm{H}\right)$; HRMS (CI) $m / z$ calcd for $\mathrm{C}_{22} \mathrm{H}_{39} \mathrm{O}_{2} \mathrm{Hg}\left(\mathrm{M}^{+}+\mathrm{H}\right) 537.2657$ found 537.2646

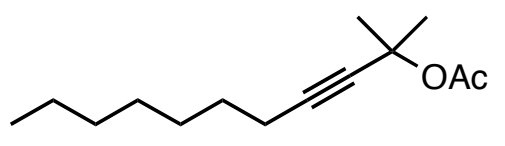

19

2-methylundec-3-yn-2-yl acetate (19): FTIR 3474, 2985, 2931, 2858, 2244, $1748 \mathrm{~cm}^{-1}$; ${ }^{1} \mathrm{H}$ NMR $\left(300 \mathrm{MHz}\right.$ in $\left.\mathrm{CDCl}_{3}\right) \delta 0.88(3 \mathrm{H}, \mathrm{t}, J=6.7 \mathrm{~Hz}), 1.24-1.40(8 \mathrm{H}, \mathrm{m}), 1.44-1.54$ $(2 \mathrm{H}, \mathrm{m}), 1.64(6 \mathrm{H}, \mathrm{s}), 2.00(3 \mathrm{H}, \mathrm{s}), 2.19(2 \mathrm{H}, \mathrm{t}, J=7.1 \mathrm{~Hz}) ;{ }^{13} \mathrm{C}$ NMR $(75 \mathrm{MHz}$ in $\left.\mathrm{CDCl}_{3}\right) \delta 13.91 \mathrm{q}, 18.54 \mathrm{t}, 21.89 \mathrm{q}, 22.46 \mathrm{t}, 28.43 \mathrm{t}, 28.59 \mathrm{t}, 28.62 \mathrm{t}, 29.15 \mathrm{q}, 29.15 \mathrm{q}, 31.60 \mathrm{t}$, 72.41s, 81.26s, 84.49s, 169.11s; MS (CI) m/z $224\left(\mathrm{M}^{+}\right)$; HRMS (CI) $\mathrm{m} / z$ calcd for $\mathrm{C}_{14} \mathrm{H}_{24} \mathrm{O}_{2}\left(\mathrm{M}^{+}\right) 224.1776$ found 224.1762

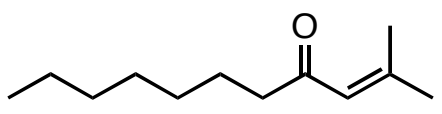

20

2-methylundec-2-en-4-one (20): FTIR 2958, 2929, 2856, 1690, $1623 \mathrm{~cm}^{-1} ;{ }^{1} \mathrm{H}$ NMR $\left(300 \mathrm{MHz}\right.$ in $\left.\mathrm{CDCl}_{3}\right) \delta 0.88(3 \mathrm{H}, \mathrm{t}, J=6.9 \mathrm{~Hz}), 1.24-1.33(8 \mathrm{H}, \mathrm{m}), 1.53-1.63(2 \mathrm{H}, \mathrm{m})$, $1.88(3 \mathrm{H}, \mathrm{d}, J=1.4 \mathrm{~Hz}), 2.14(3 \mathrm{H}, \mathrm{d}, J=1.4 \mathrm{~Hz}), 2.39(2 \mathrm{H}, \mathrm{t}, J=7.6 \mathrm{~Hz}), 6.07(1 \mathrm{H}, \mathrm{m})$; ${ }^{13} \mathrm{C} \mathrm{NMR}\left(75 \mathrm{MHz}\right.$ in $\mathrm{CDCl}_{3}$ ) $\delta 14.03 \mathrm{q}, 20.60 \mathrm{q}, 22.59 \mathrm{t}, 24.27 \mathrm{t}, 27.58 \mathrm{q}, 29.11 \mathrm{t}, 29.26 \mathrm{t}$, 31.69t, 44.32t, 123.80d, 154.57s, 201.31s; MS (CI) m/z $183\left(\mathrm{M}^{+}+\mathrm{H}\right)$; HRMS (CI) $m / z$ calcd for $\mathrm{C}_{12} \mathrm{H}_{23} \mathrm{O}\left(\mathrm{M}^{+}+\mathrm{H}\right) 183.1749$ found 183.1735 


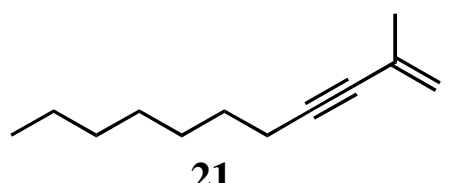

21

2-methylundec-1-en-3-yne (21): FTIR 3096, 2953, 2931, 2857, 2225, $1615 \mathrm{~cm}^{-1} ;{ }^{1} \mathrm{H}$ NMR (300 MHz in $\left.\mathrm{CDCl}_{3}\right) \delta 0.89(3 \mathrm{H}, \mathrm{t}, J=6.7 \mathrm{~Hz}), 1.24-1.44(8 \mathrm{H}, \mathrm{m}), 1.48-1.58(2 \mathrm{H}$, m), $1.87(3 \mathrm{H}, \mathrm{dd}, J=1.5,1.1 \mathrm{~Hz}), 2.29(2 \mathrm{H}, \mathrm{t}, J=7.0 \mathrm{~Hz}), 5.13(1 \mathrm{H}, \mathrm{m}), 5.19(1 \mathrm{H}, \mathrm{m})$; ${ }^{13} \mathrm{C}$ NMR $\left(75 \mathrm{MHz}\right.$ in $\left.\mathrm{CDCl}_{3}\right) \delta 14.08 \mathrm{q}, 19.28 \mathrm{t}, 22.63 \mathrm{t}, 23.87 \mathrm{q}, 28.78 \mathrm{t}, 28.83 \mathrm{t}, 28.86 \mathrm{t}$, 31.76t, 81.82s, 89.54s, 120.21t, 127.40s; MS (CI) $m / z 165\left(\mathrm{M}^{+}+\mathrm{H}\right)$; HRMS (CI) $m / z$

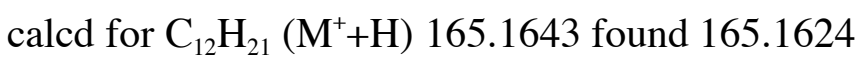

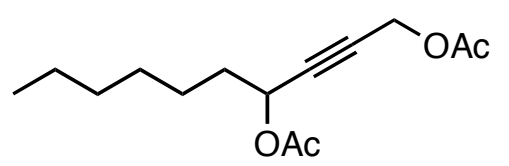

22

dec-2-yne-1,4-diyl diacetate (22): FTIR 3473, 2953, 2932, 2860, $1759 \mathrm{~cm}^{-1}$; ${ }^{1} \mathrm{H}$ NMR $\left(300 \mathrm{MHz}\right.$ in $\left.\mathrm{CDCl}_{3}\right) \delta 0.89(3 \mathrm{H}, \mathrm{t}, J=6.7 \mathrm{~Hz}), 1.26-1.46(8 \mathrm{H}, \mathrm{m}), 1.72-1.80(2 \mathrm{H}, \mathrm{m})$, $2.08(3 \mathrm{H}, \mathrm{s}), 2.09(3 \mathrm{H}, \mathrm{s}), 4.70(2 \mathrm{H}, \mathrm{d}, J=1.7 \mathrm{~Hz}), 5.39(1 \mathrm{H}, \mathrm{tt}, J=6.7,1.7 \mathrm{~Hz}) ;{ }^{13} \mathrm{C}$ NMR (75 MHz in $\left.\mathrm{CDCl}_{3}\right) \delta 13.79 \mathrm{q}, 20.41 \mathrm{q}, 20.68 \mathrm{q}, 22.30 \mathrm{t}, 24.67 \mathrm{t}, 28.51 \mathrm{t}, 31.39 \mathrm{t}$, 34.32t, 51.87t, 63.61d, 79.13s, 84.05s, 169.57s, 169.77s; MS (CI) m/z $253\left(\mathrm{M}^{+}-\mathrm{H}\right)$; HRMS (CI) $m / z$ calcd for $\mathrm{C}_{14} \mathrm{H}_{21} \mathrm{O}_{4}\left(\mathrm{M}^{+}-\mathrm{H}\right) 253.1440$ found 253.1449

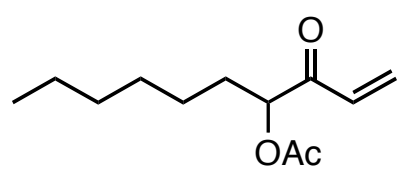

23

3-oxodec-1-en-4-yl acetate (23): FTIR 2953, 2930, 2860, 1747, 1713, $1615 \mathrm{~cm}^{-1} ;{ }^{1} \mathrm{H}$ NMR $\left(300 \mathrm{MHz}\right.$ in $\left.\mathrm{CDCl}_{3}\right) \delta 0.88(3 \mathrm{H}, \mathrm{t}, J=6.7 \mathrm{~Hz}), 1.24-1.44(8 \mathrm{H}, \mathrm{m}), 1.72-1.81(2 \mathrm{H}$, m), $2.15(3 \mathrm{H}, \mathrm{s}), 5.21(1 \mathrm{H}, \mathrm{dd}, J=7.6,5.3 \mathrm{~Hz}), 5.84(1 \mathrm{H}, \mathrm{dd}, J=10.2,1.9 \mathrm{~Hz}), 6.38$ $(1 \mathrm{H}, \mathrm{dd}, J=17.5,1.9 \mathrm{~Hz}), 6.51(1 \mathrm{H}, \mathrm{dd}, J=17.5,10.2 \mathrm{~Hz}) ;{ }^{13} \mathrm{C}$ NMR $(75 \mathrm{MHz}$ in $\left.\mathrm{CDCl}_{3}\right) \delta 13.85 \mathrm{q}, 20.48 \mathrm{q}, 22.37 \mathrm{t}, 25.00 \mathrm{t}, 28.75 \mathrm{t}, 30.46 \mathrm{t}, 31.38 \mathrm{t}, 77.18 \mathrm{~d}, 129.74 \mathrm{t}$, 131.66d, 170.31s, 196.05s; MS (CI) $m / z 213\left(\mathrm{M}^{+}+\mathrm{H}\right)$; HRMS (CI) $m / z$ calcd for $\mathrm{C}_{12} \mathrm{H}_{21} \mathrm{O}_{3}\left(\mathrm{M}^{+}+\mathrm{H}\right) 213.1491$ found 213.1499 


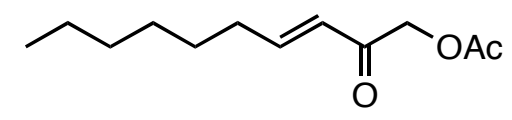

24

(E)-2-oxodec-3-enyl acetate (24): FTIR 2958, 2930, 2858, 1753, 1713, 1696, 1634 $\mathrm{cm}^{-1} ;{ }^{1} \mathrm{H}$ NMR $\left(600 \mathrm{MHz}\right.$ in $\left.\mathrm{CDCl}_{3}\right) \delta 0.89(3 \mathrm{H}, \mathrm{t}, J=7.0 \mathrm{~Hz}), 1.25-1.35(6 \mathrm{H}, \mathrm{m}), 1.47$ $(2 \mathrm{H}$, quintet, $J=7.4 \mathrm{~Hz}) 2.19(3 \mathrm{H}, \mathrm{s}), 2.24(2 \mathrm{H}, \mathrm{ddt}, J=7.9,1.5,7.1 \mathrm{~Hz}), 4.84(2 \mathrm{H}, \mathrm{s})$, $6.15(1 \mathrm{H}, \mathrm{dt}, J=16.0,1.5 \mathrm{~Hz}), 6.95(1 \mathrm{H}, \mathrm{dt}, J=16.0,6.9 \mathrm{~Hz}) ;{ }^{13} \mathrm{C} \mathrm{NMR}(75 \mathrm{MHz}$ in $\left.\mathrm{CDCl}_{3}\right) \delta 13.99 \mathrm{q}, 20.50 \mathrm{q}, 22.47 \mathrm{t}, 27.83 \mathrm{t}, 28.80 \mathrm{t}, 31.52 \mathrm{t}, 32.69 \mathrm{t}, 66.82 \mathrm{t}, 125.95 \mathrm{~d}$, 149.51d, 170.28s, 192.29s; MS (CI) $m / z 213\left(\mathrm{M}^{+}+\mathrm{H}\right)$; HRMS (CI) $m / z$ calcd for $\mathrm{C}_{12} \mathrm{H}_{21} \mathrm{O}_{3}\left(\mathrm{M}^{+}+\mathrm{H}\right) 213.1490$ found 213.1483

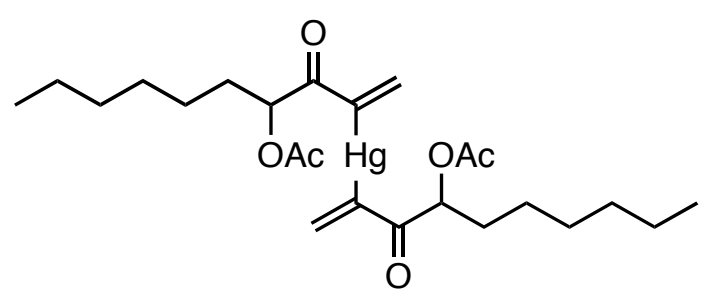

25

bis (4-acetoxy-3-oxodec-1-en-2-yl) mercury (25): ${ }^{1} \mathrm{H}$ NMR $\left(600 \mathrm{MHz}\right.$ in $\left.\mathrm{CDCl}_{3}\right) \delta$ $0.88(6 \mathrm{H}, \mathrm{t}, J=7.1 \mathrm{~Hz}), 1.35-1.44(12 \mathrm{H}, \mathrm{m}), 1.73-1.83(4 \mathrm{H}, \mathrm{m}), 2.12(6 \mathrm{H}, \mathrm{s}), 5.42(2 \mathrm{H}$, $\mathrm{dd}, J=8.7,4.4 \mathrm{~Hz}), 6.08(2 \mathrm{H}, \mathrm{d}, J=0.8 \mathrm{~Hz}), 6.72(2 \mathrm{H}, \mathrm{brs}) ; \mathrm{MS}(\mathrm{CI}) \mathrm{m} / z 625\left(\mathrm{M}^{+}+\mathrm{H}\right)$; HRMS (CI) $m / z$ calcd for $\mathrm{C}_{24} \mathrm{H}_{39} \mathrm{O}_{6} \mathrm{Hg}\left(\mathrm{M}^{+}+\mathrm{H}\right) 625.2453$ found 625.2456<smiles>CCCCCCC=C(N/C(=C\CCCCCC)C(=O)OC(C)=O)C(=O)COC(C)=O</smiles>

26

bis $\left((Z)\right.$-1-acetoxy-2-oxodec-3-en-3-yl) mercury (26): ${ }^{1} \mathrm{H} \mathrm{NMR}\left(600 \mathrm{MHz}\right.$ in $\left.\mathrm{CDCl}_{3}\right) \delta$ $0.89(6 \mathrm{H}, \mathrm{t}, J=6.9 \mathrm{~Hz}), 1.24-1.36(12 \mathrm{H}, \mathrm{m}), 1.51(4 \mathrm{H}$, quintet, $J=7.1 \mathrm{~Hz}), 2.18(6 \mathrm{H}, \mathrm{s})$, $2.41(4 \mathrm{H}, \mathrm{dt}, J=7.4,7.1 \mathrm{~Hz}), 5.02(4 \mathrm{H}, \mathrm{s}), 7.77(2 \mathrm{H}, \mathrm{t}, J=7.1 \mathrm{~Hz})$; MS (CI) $\mathrm{m} / z .625$ $\left(\mathrm{M}^{+}+\mathrm{H}\right)$; HRMS (CI) $m / z$ calcd for $\mathrm{C}_{24} \mathrm{H}_{39} \mathrm{O}_{6} \mathrm{Hg}\left(\mathrm{M}^{+}+\mathrm{H}\right) 625.2453$ found 625.2456 
arow

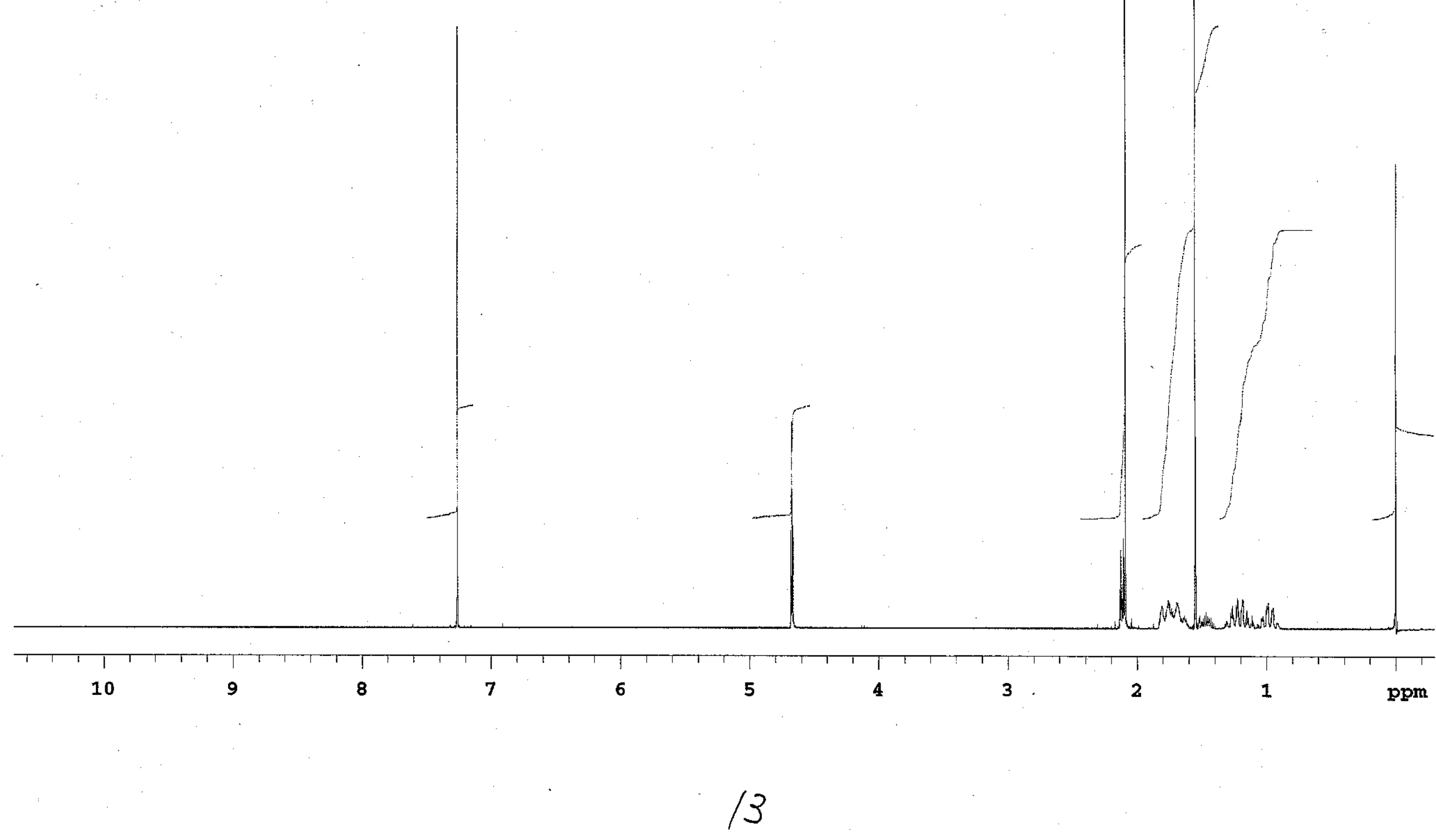



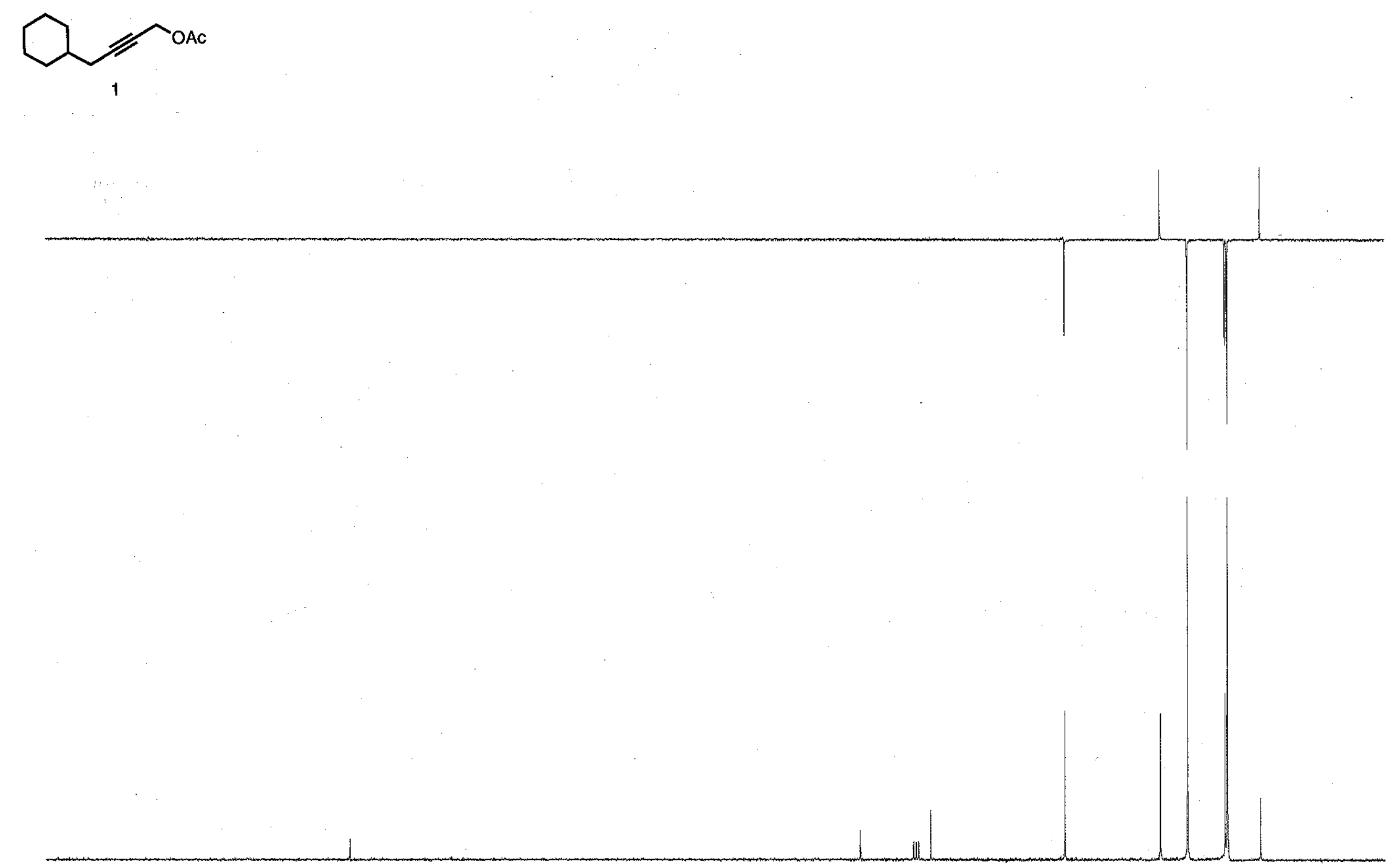

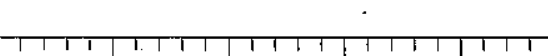

200

180

160

140

120

100

80

60

40

20

ppm. 
ain

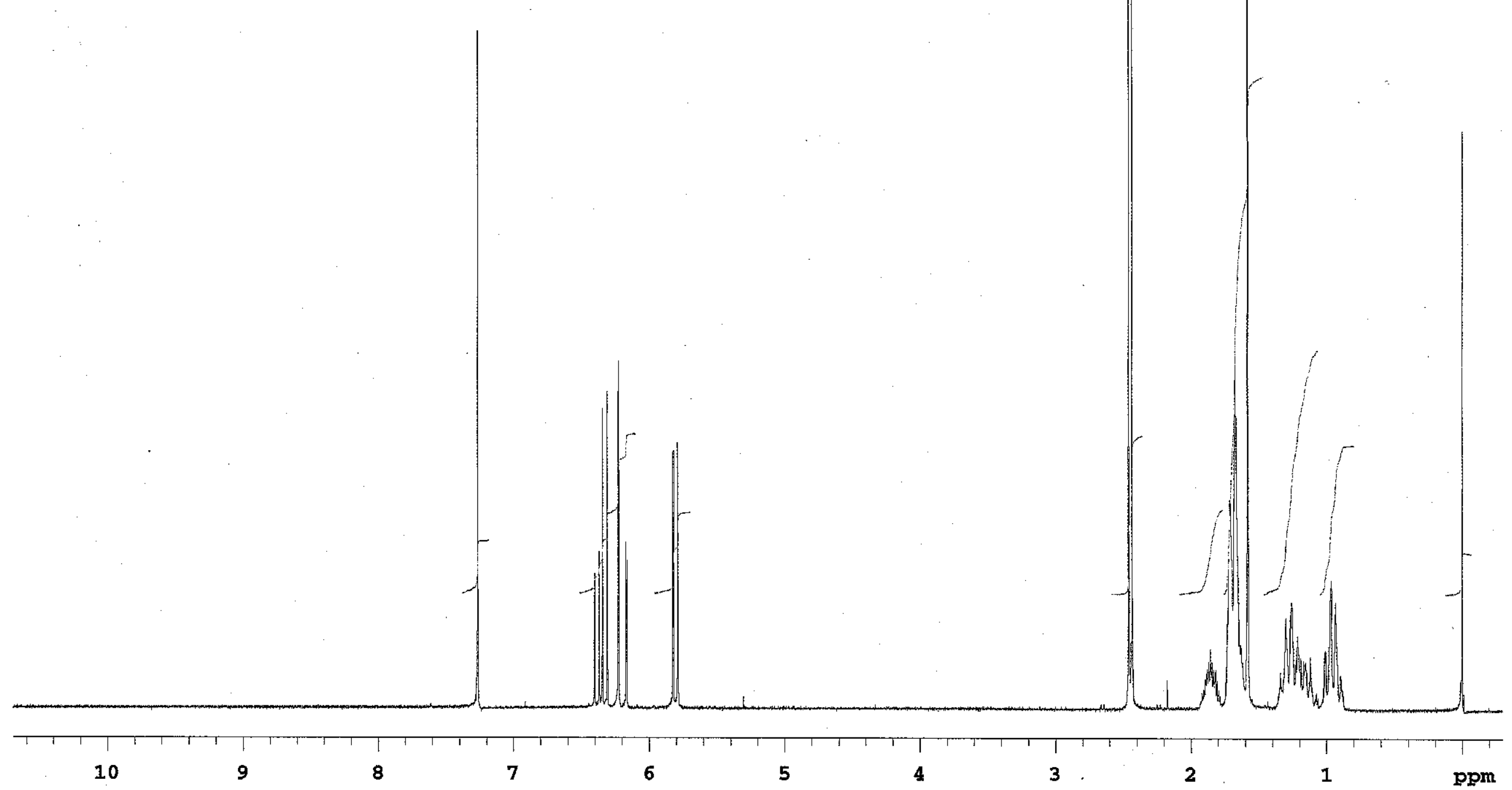




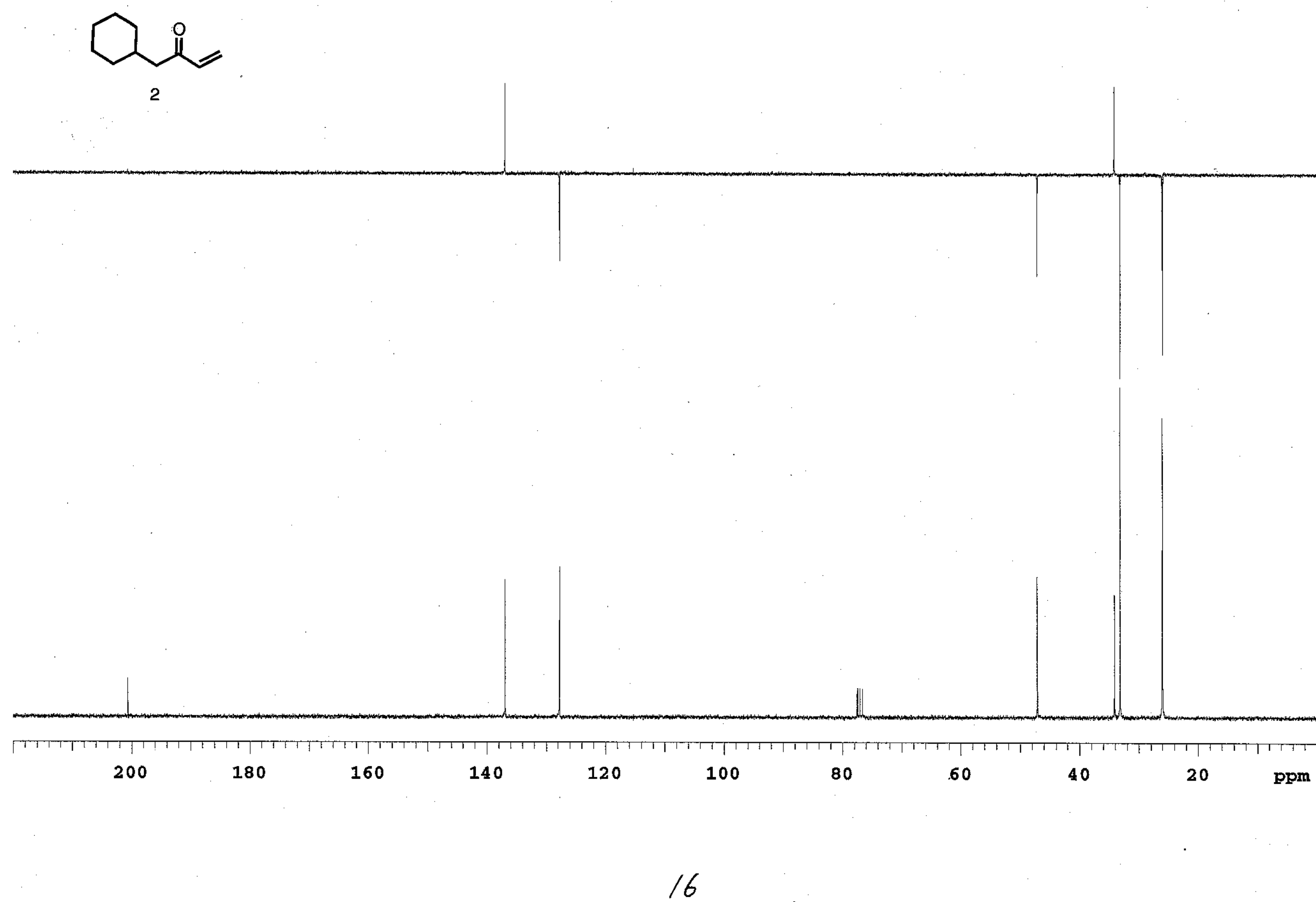




$$
\text { "twe }
$$




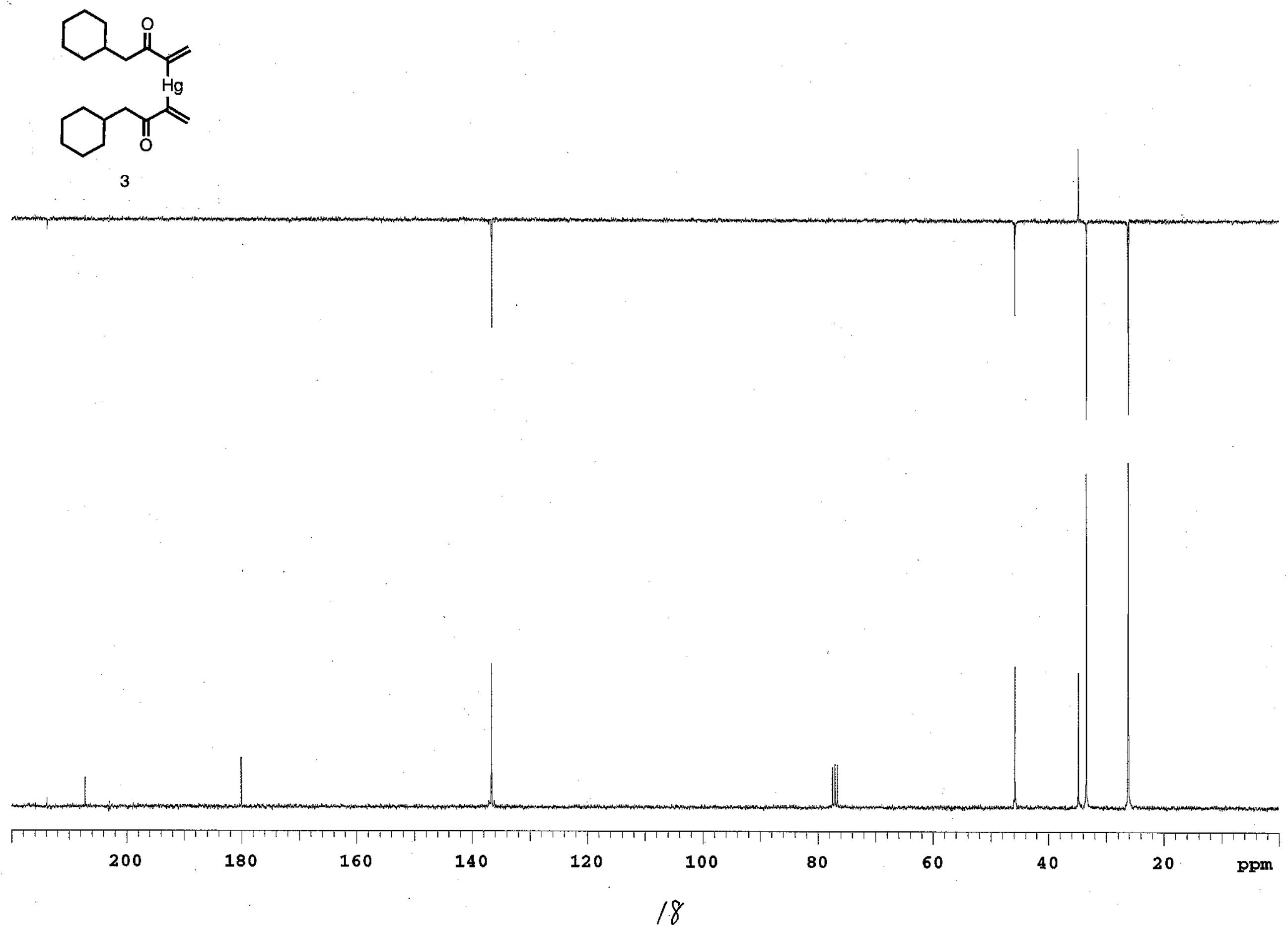


Din

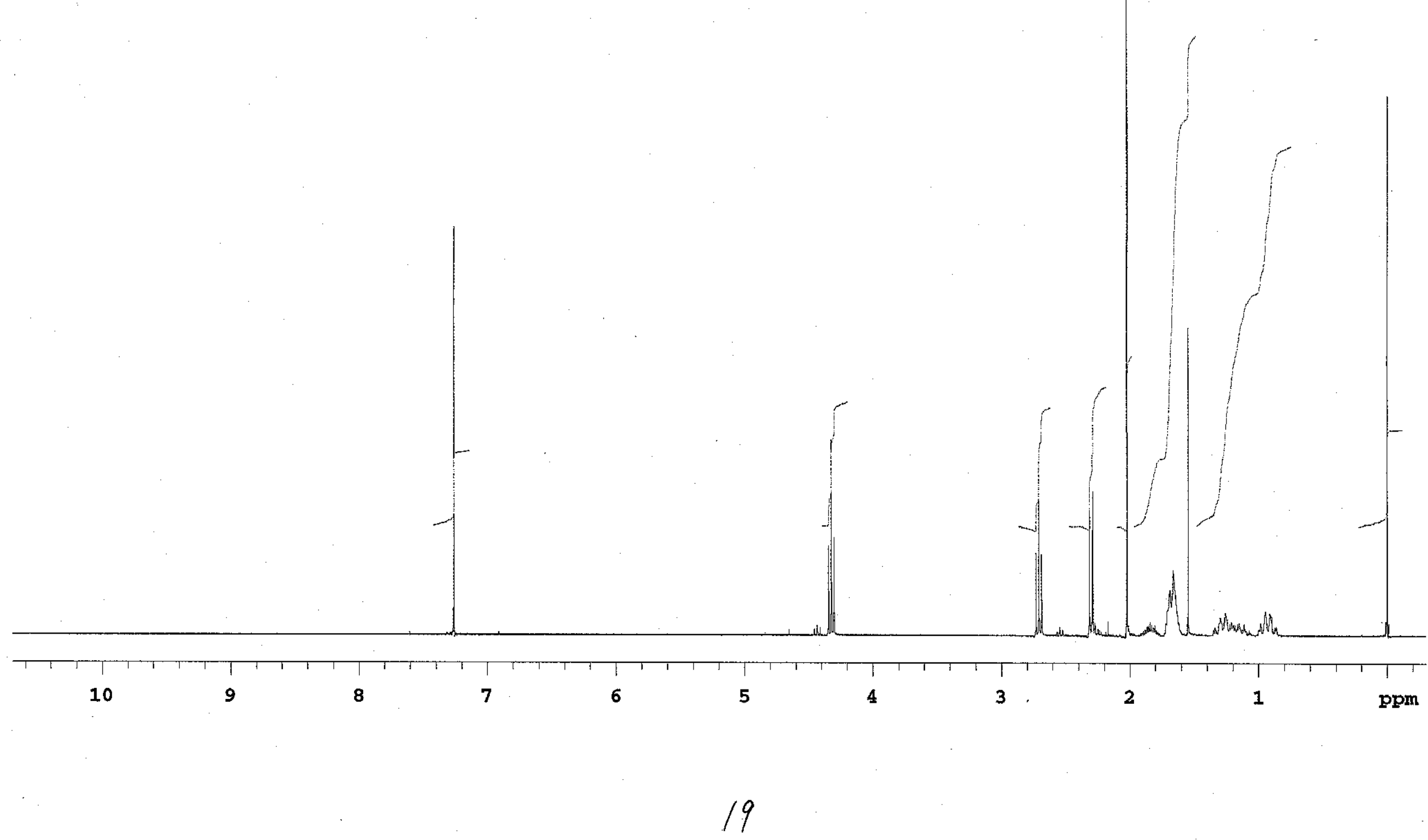




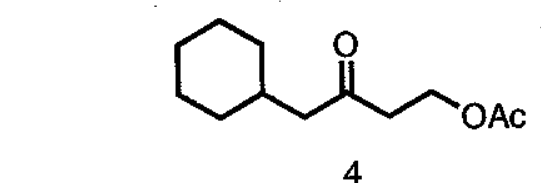

4
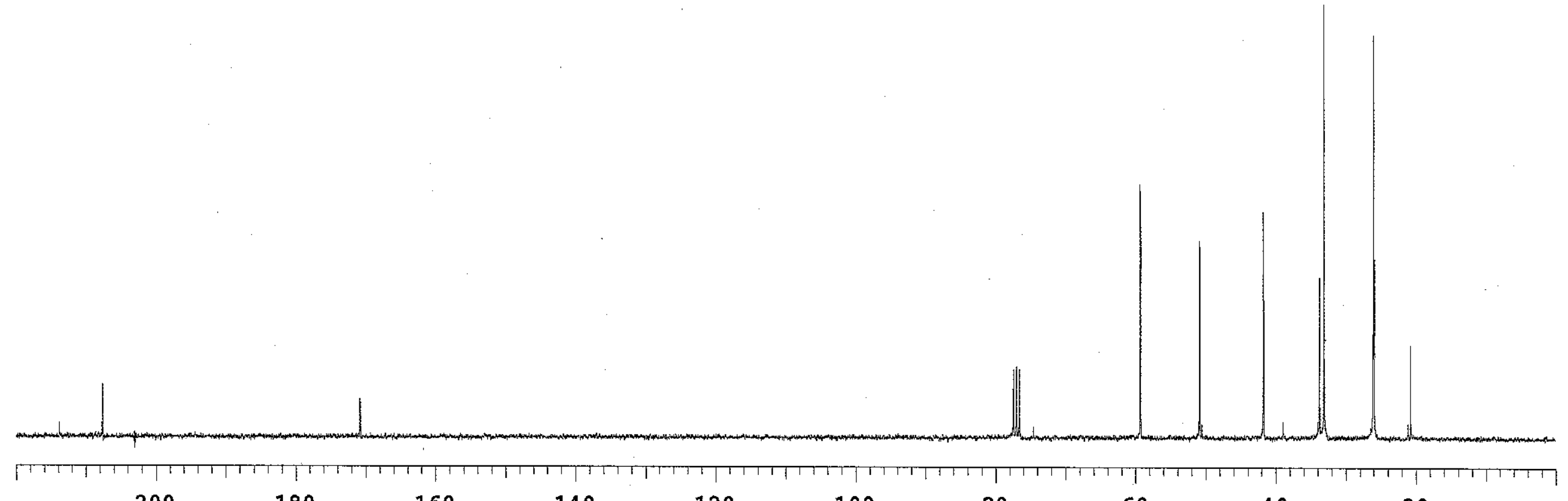
arow

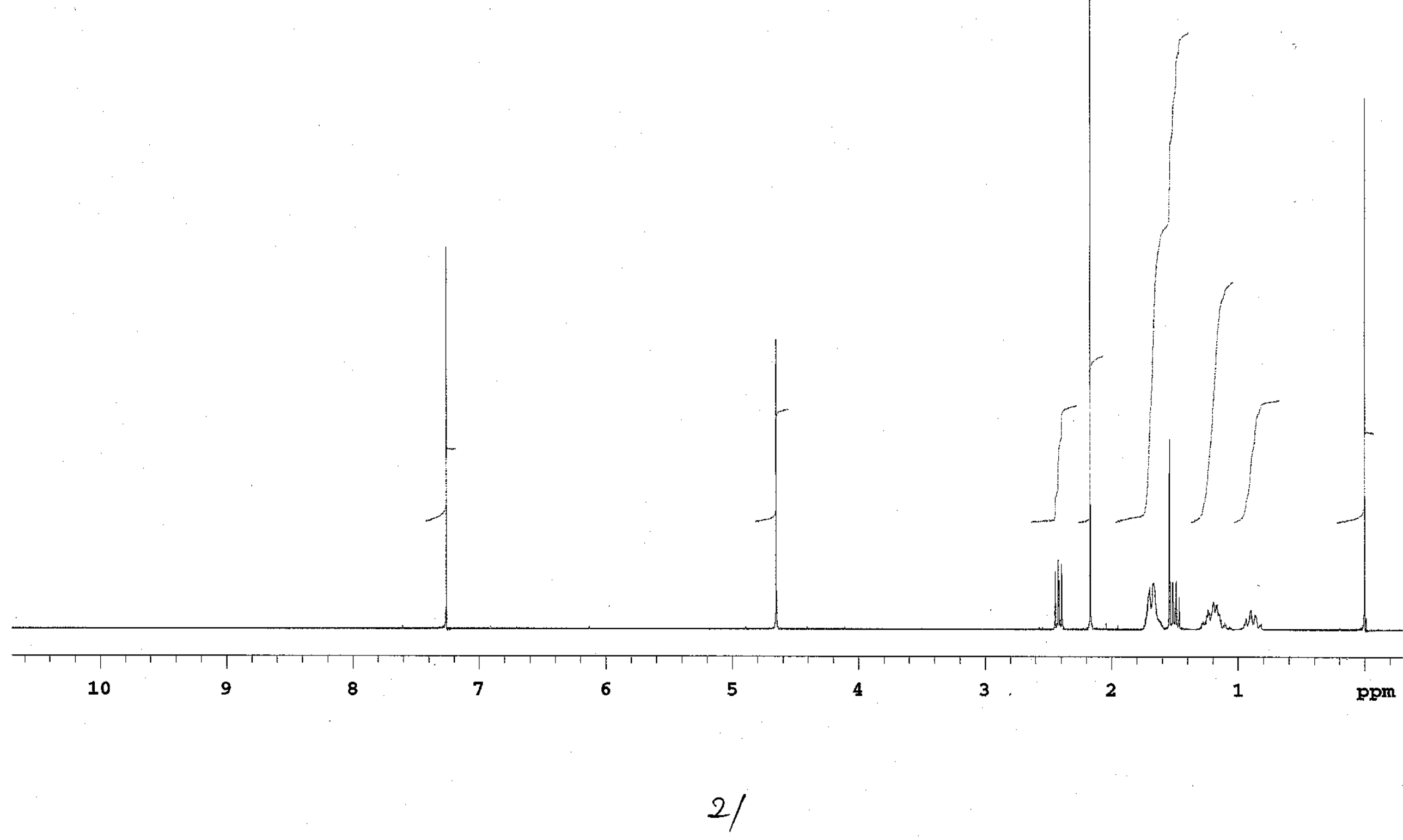




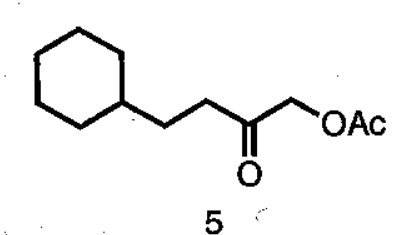

5

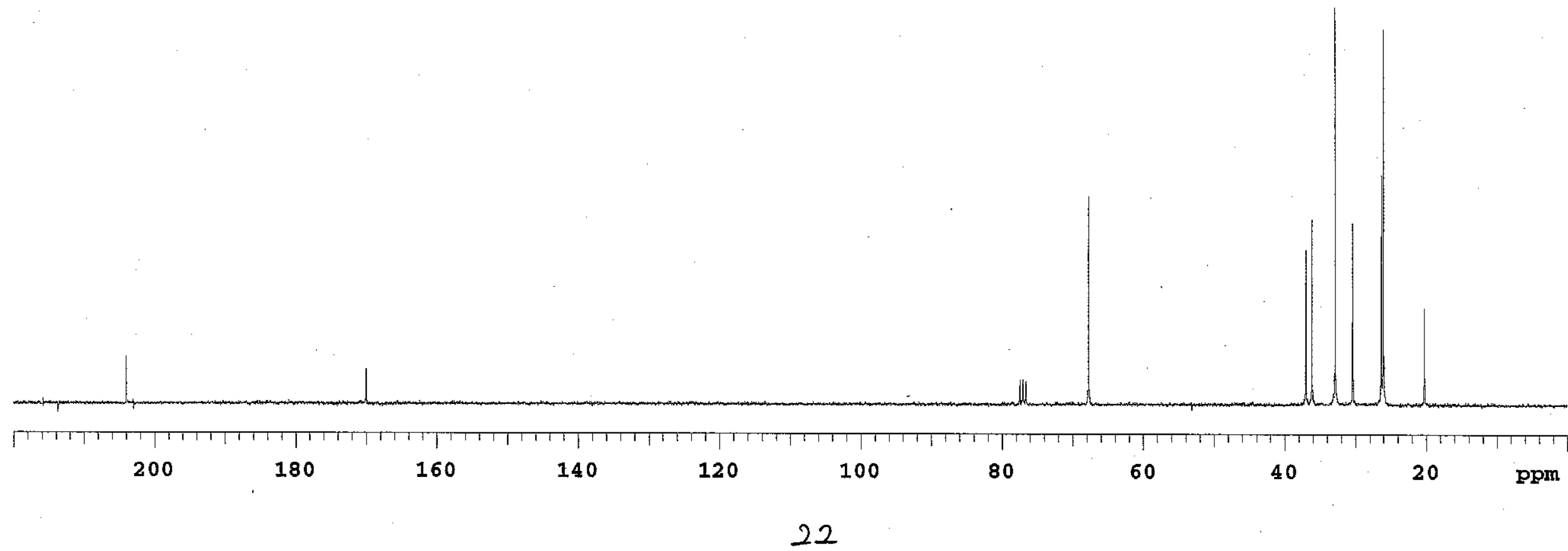




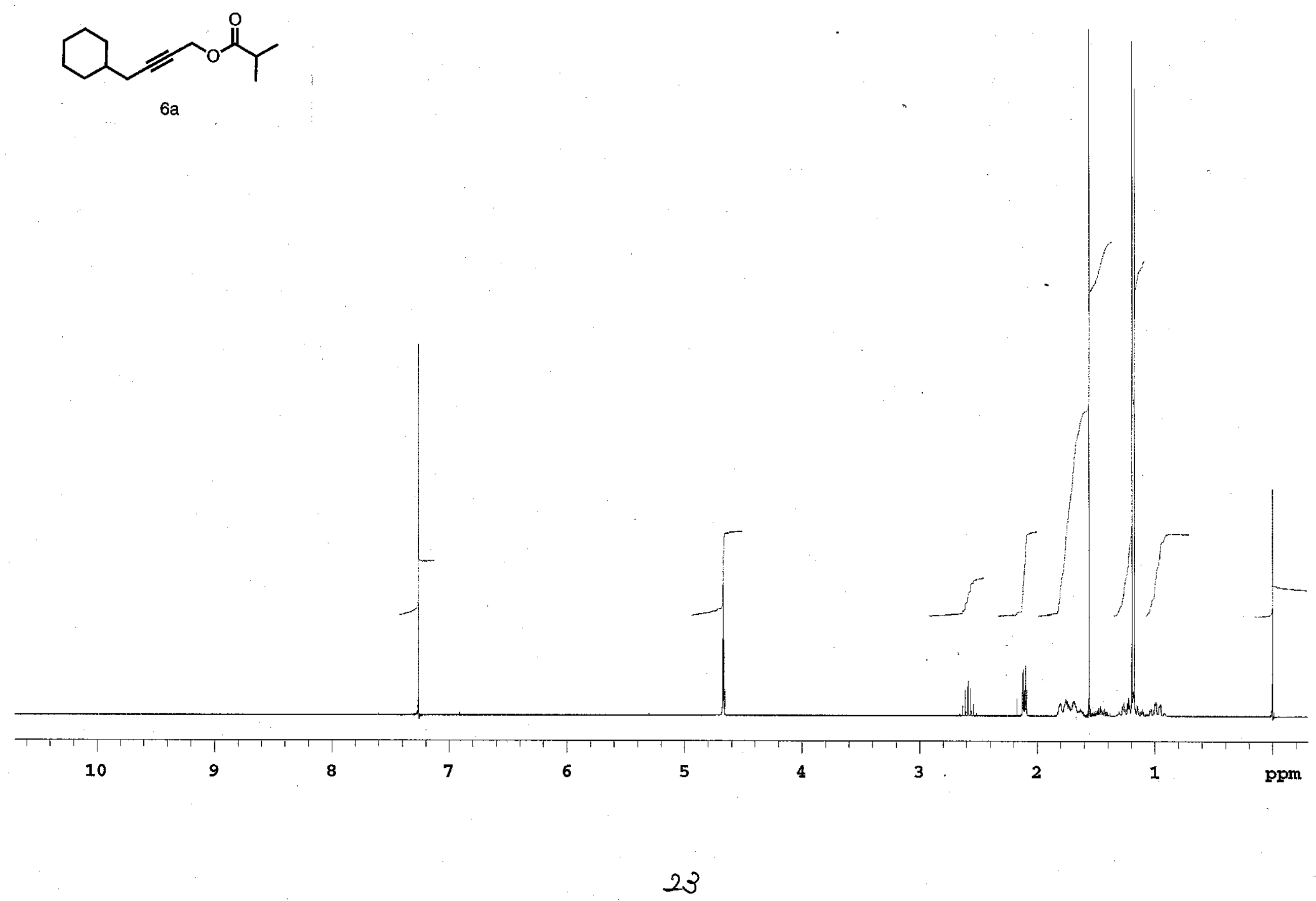




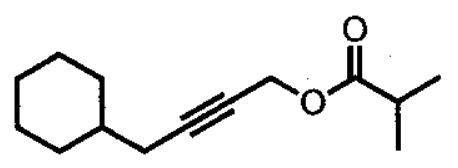

$6 a$

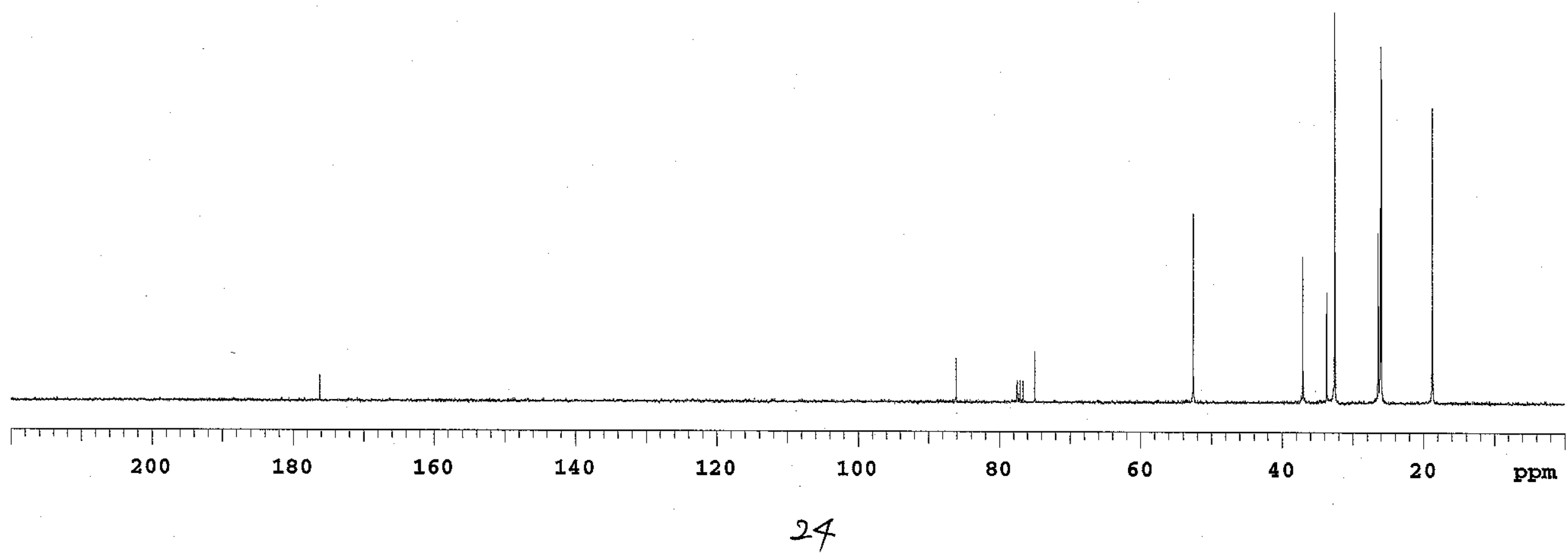


arok

$6 b$

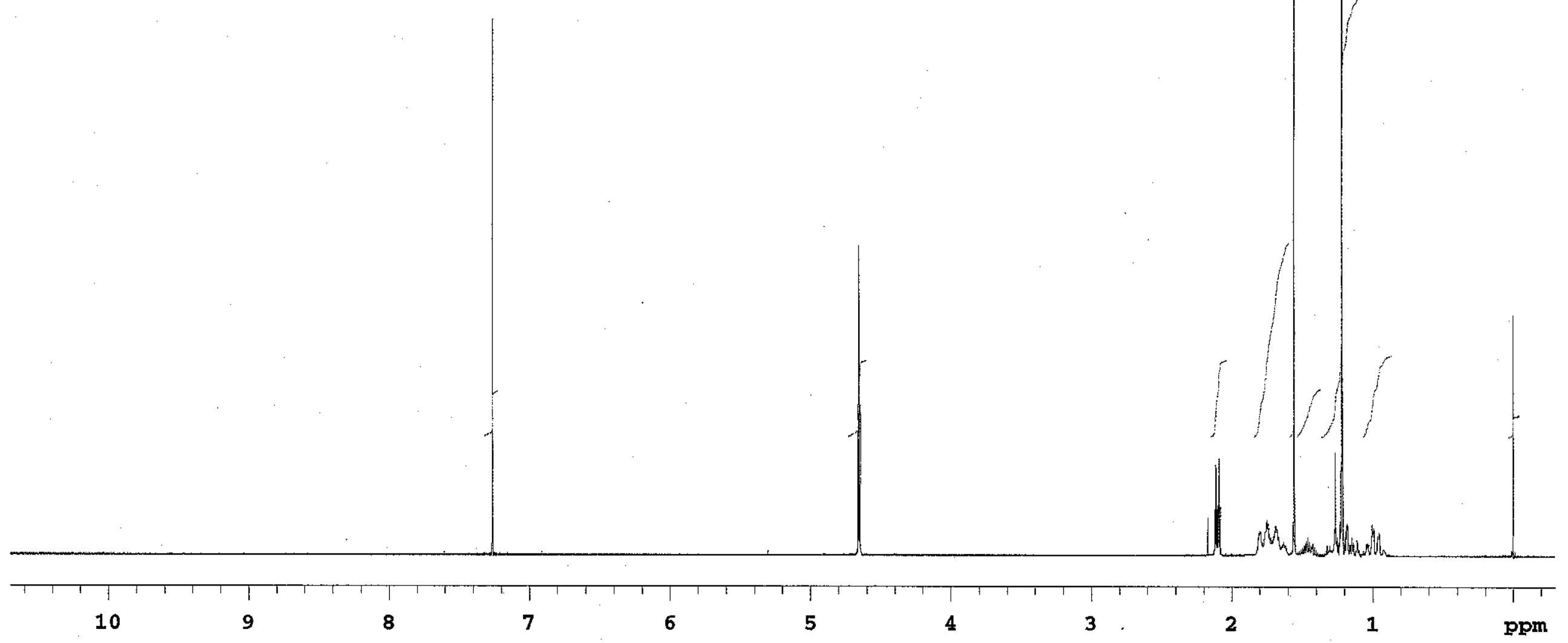




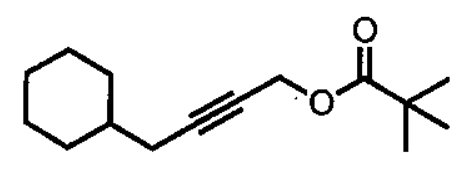

$6 b$

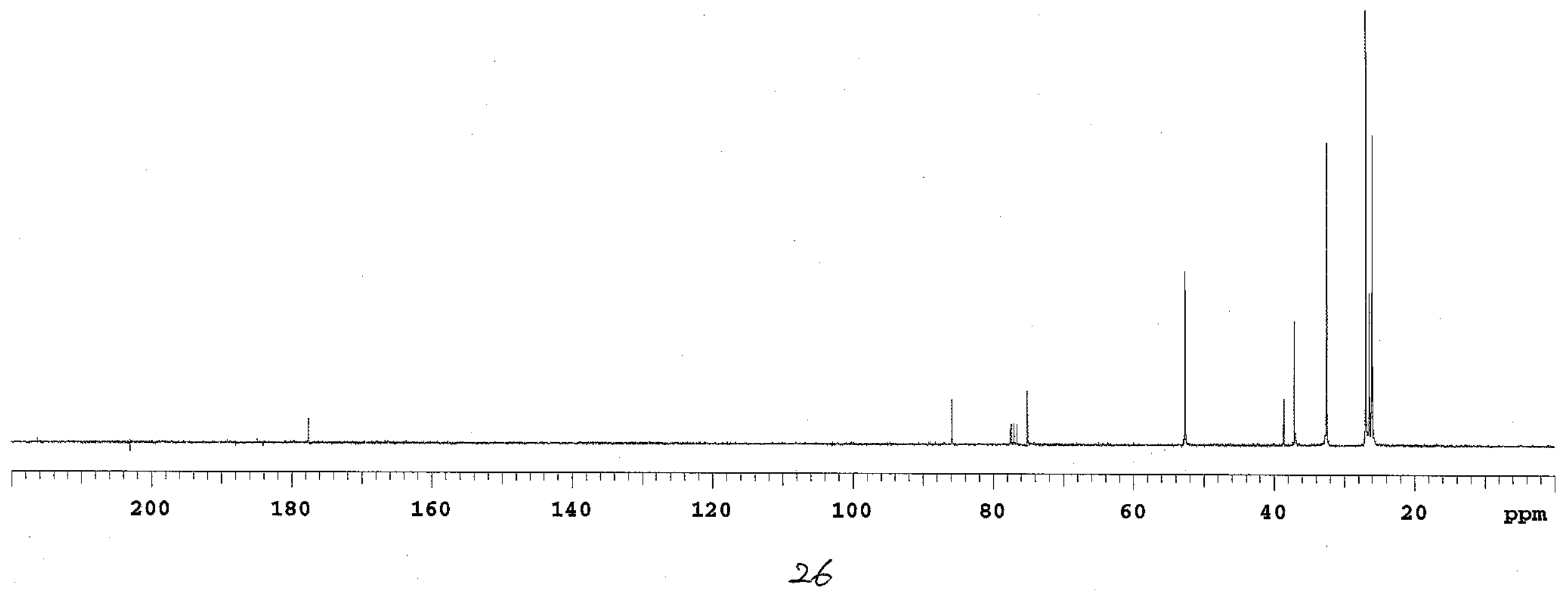




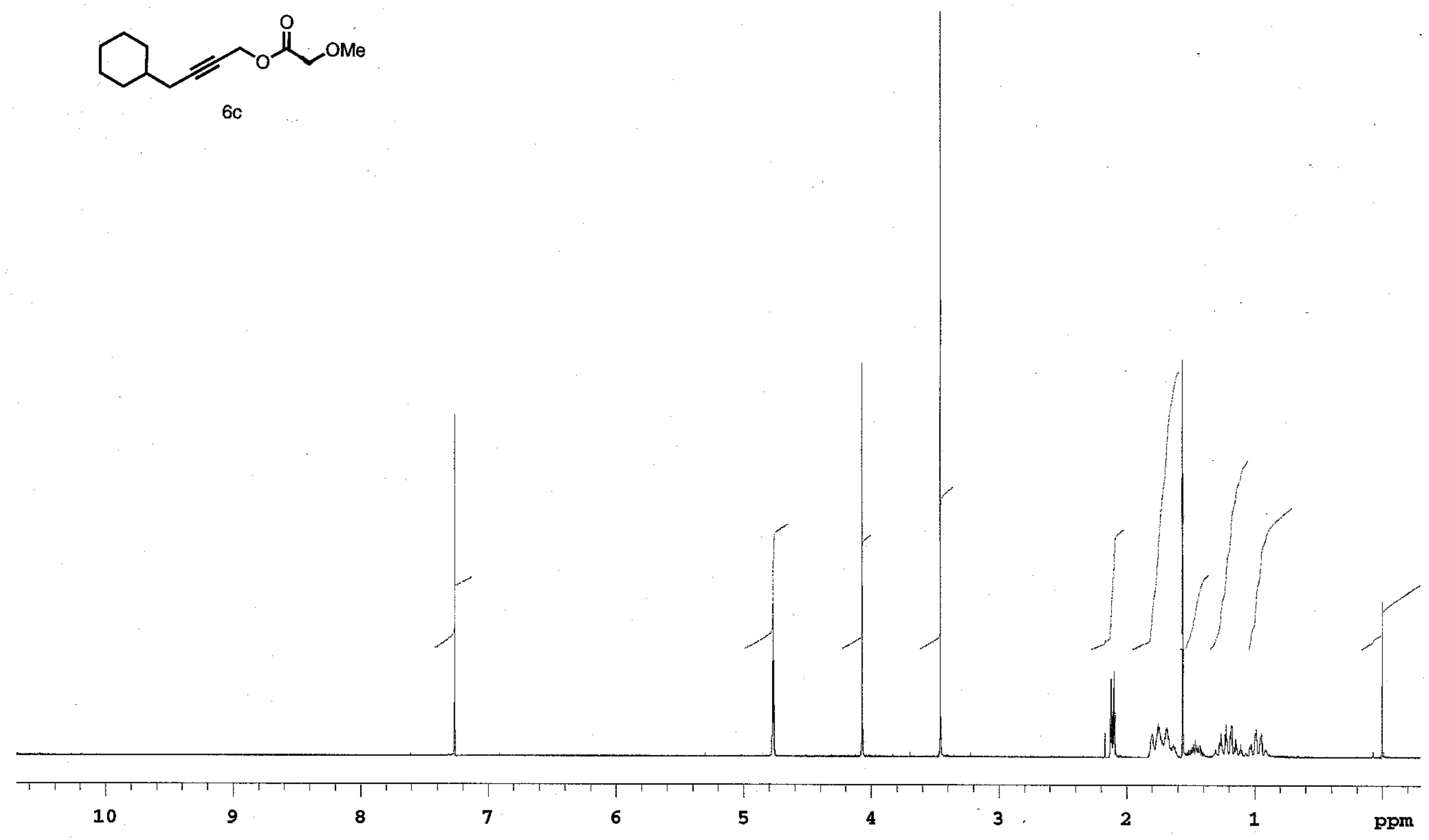

27 


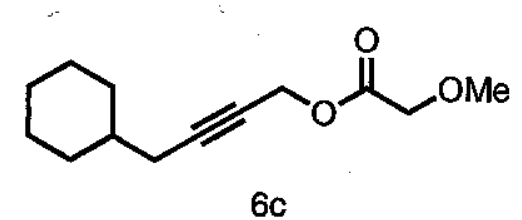

$6 c$
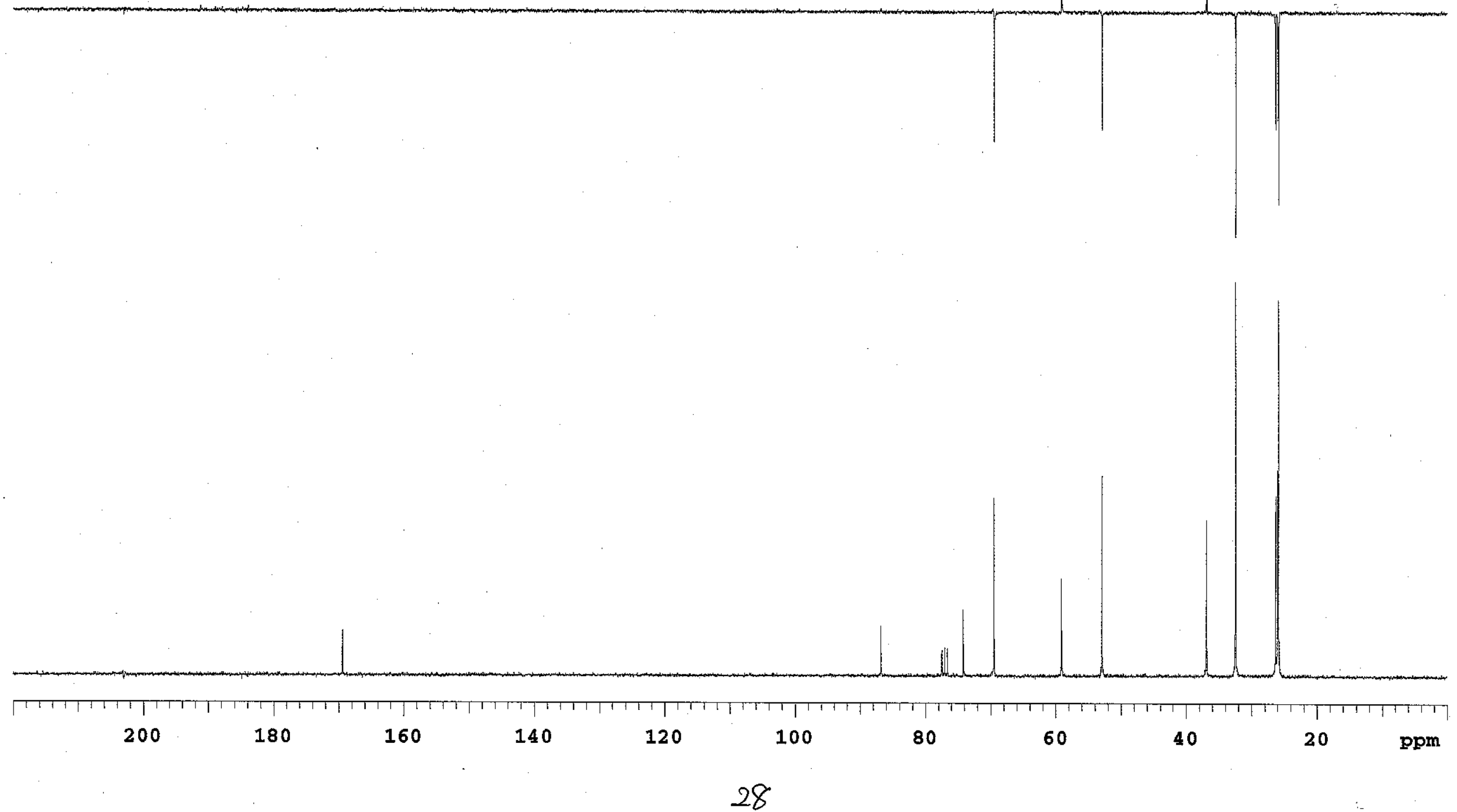
and

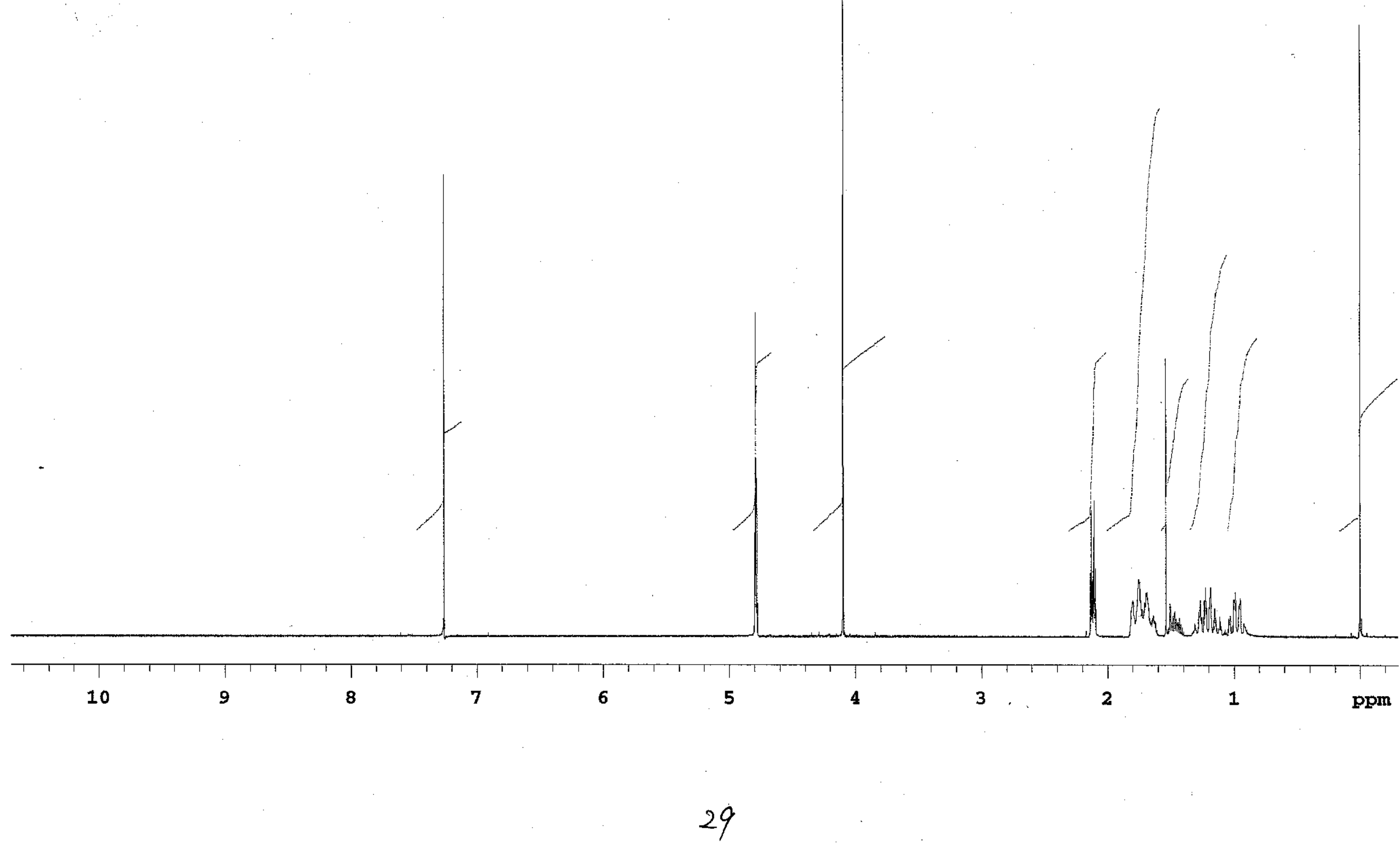


ardo
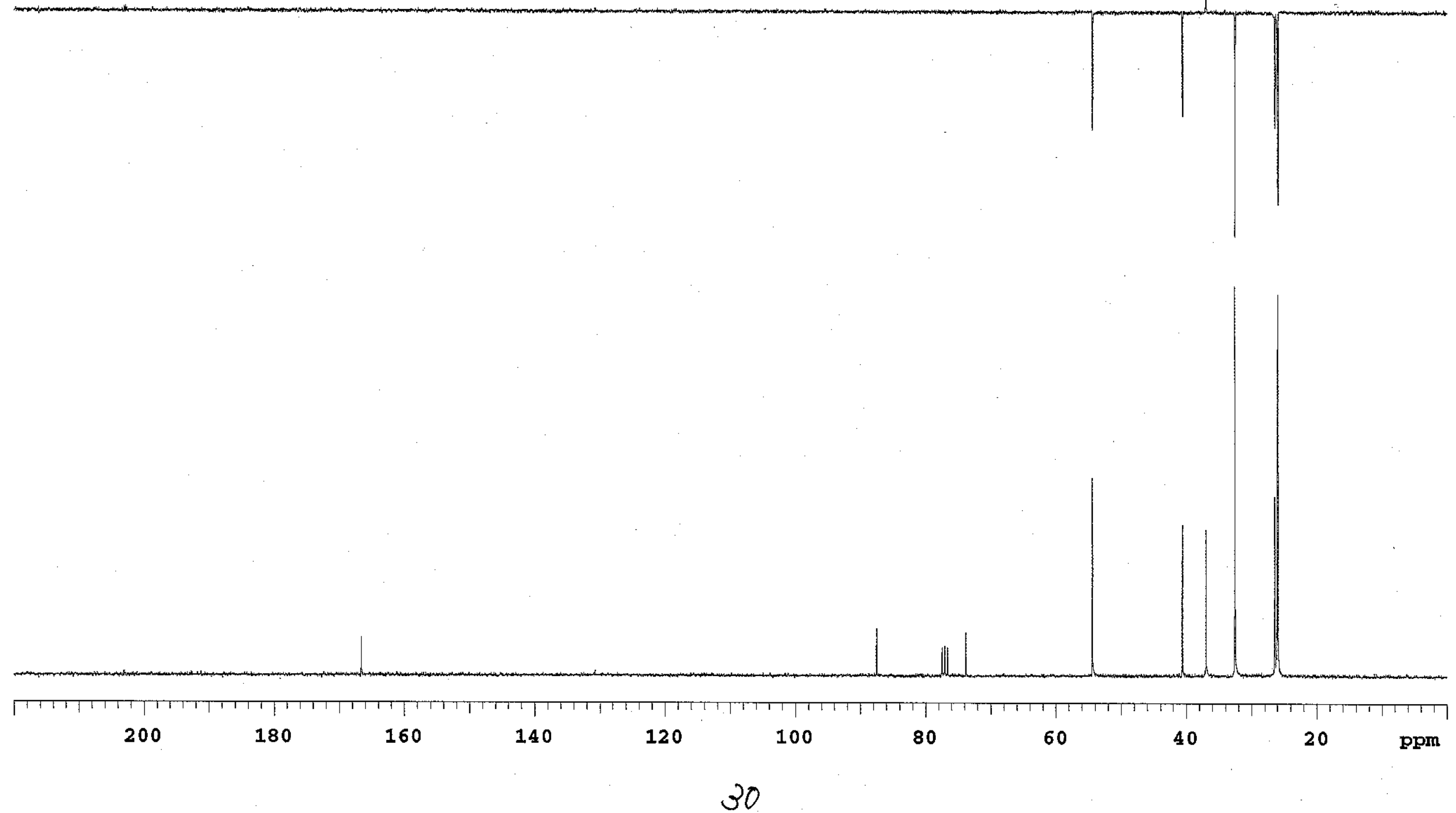


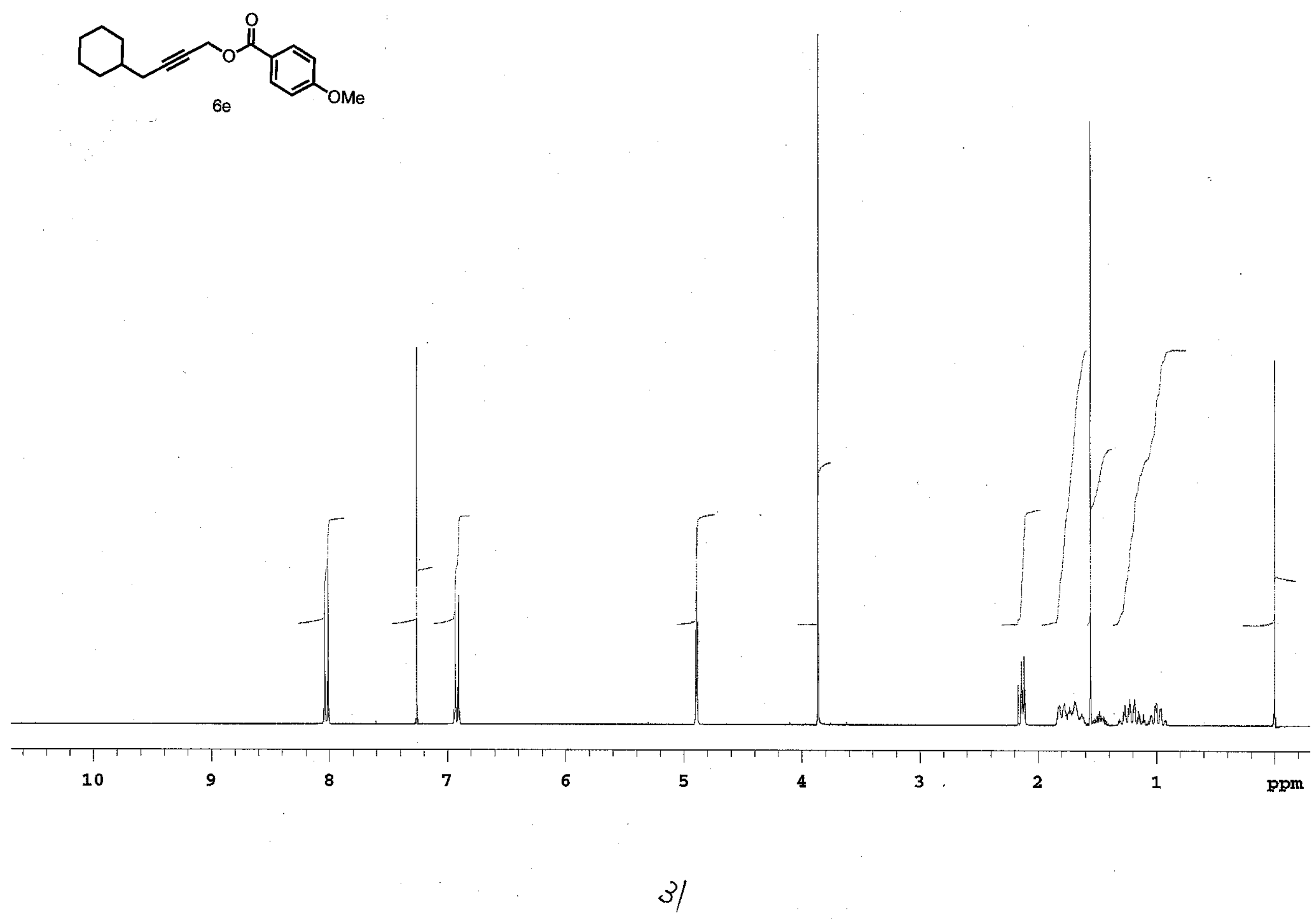




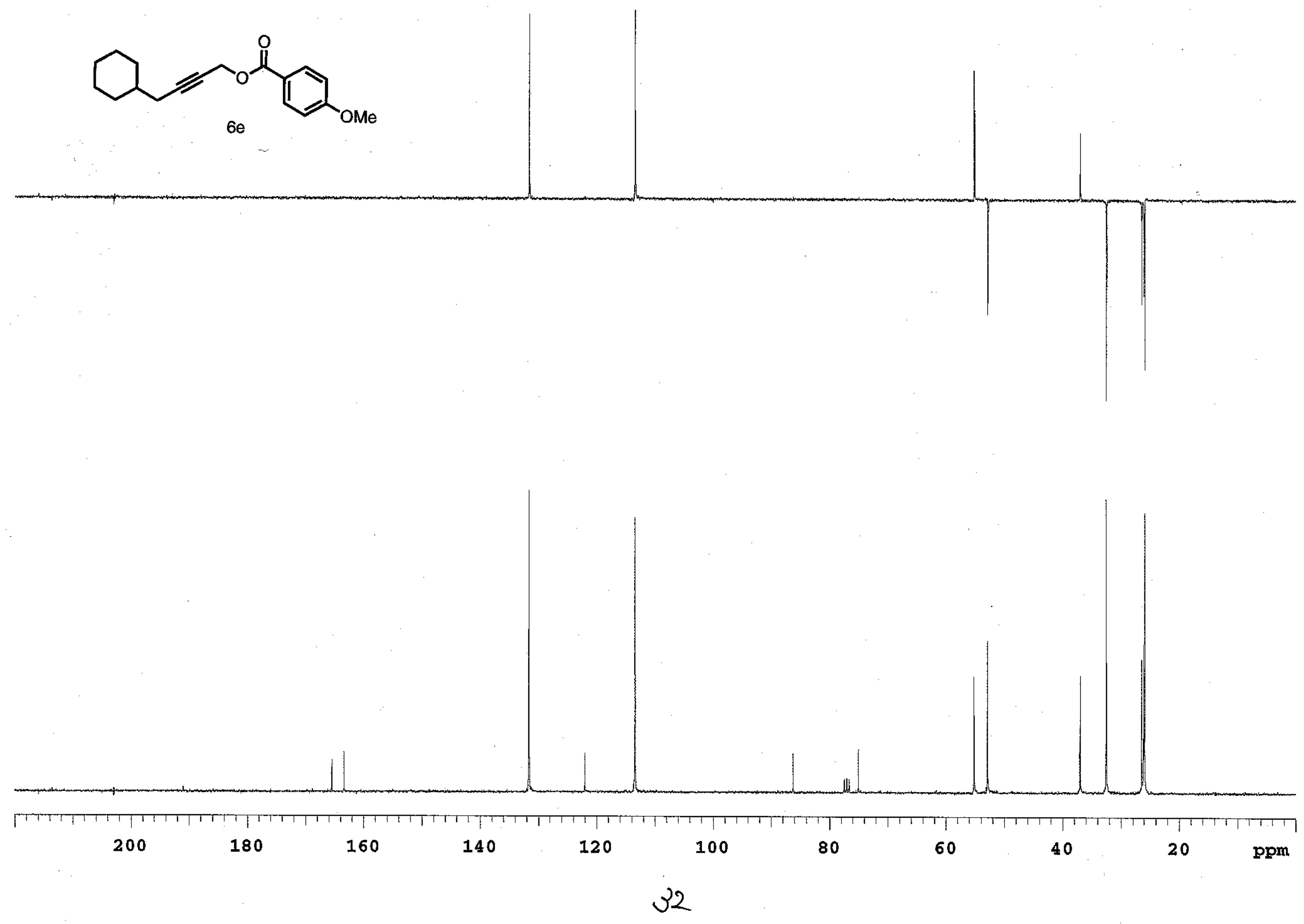




$$
116
$$



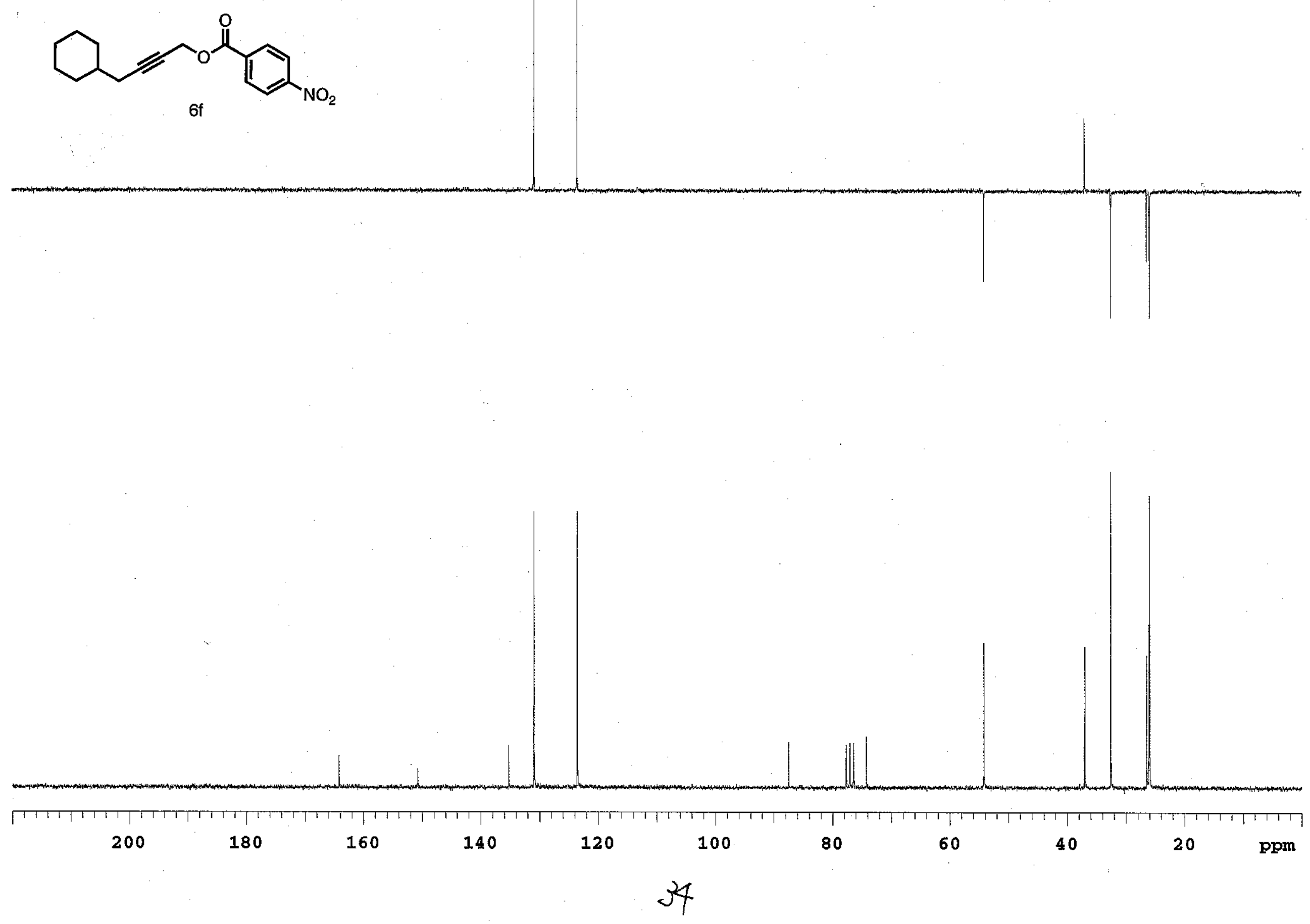


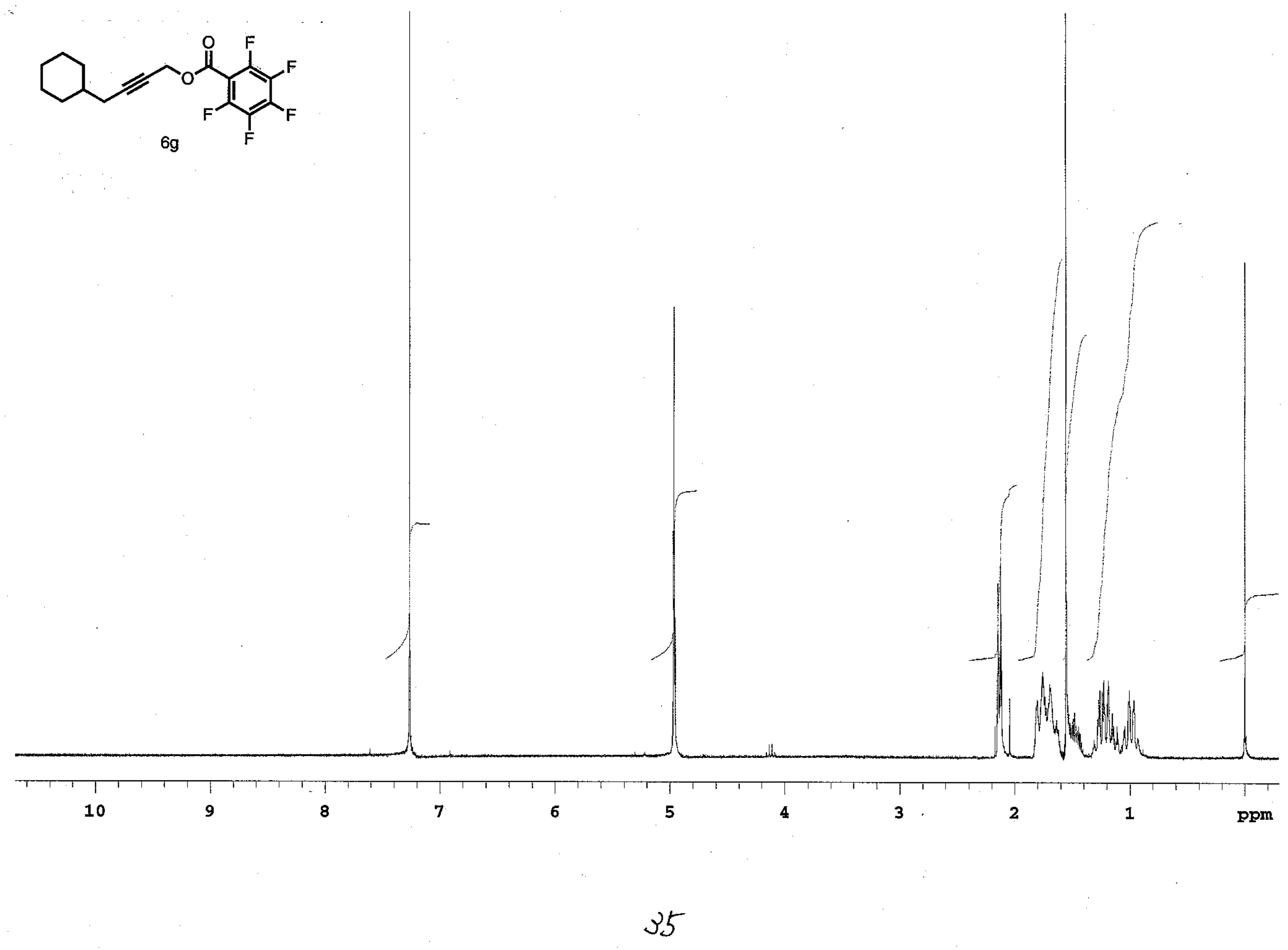



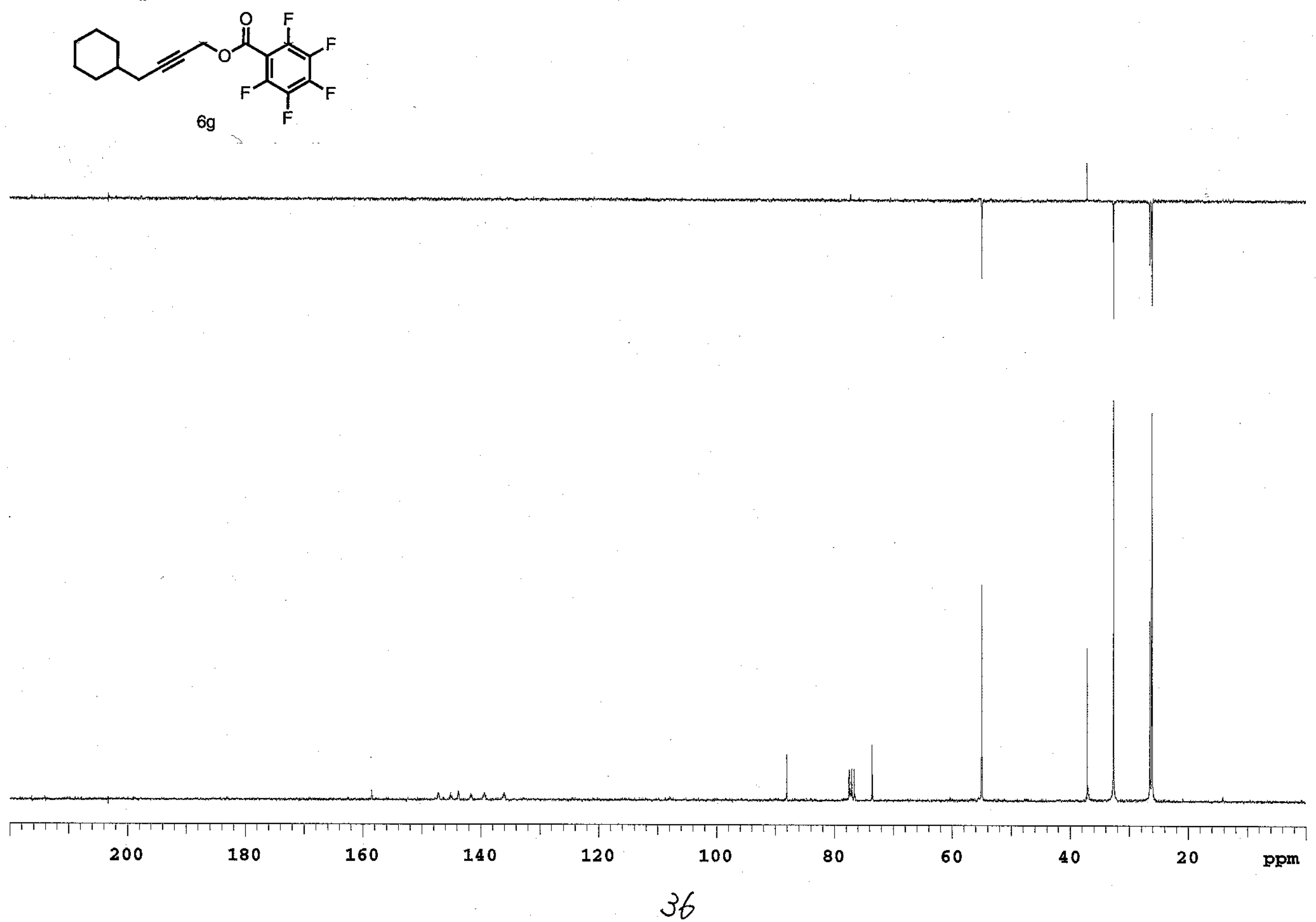


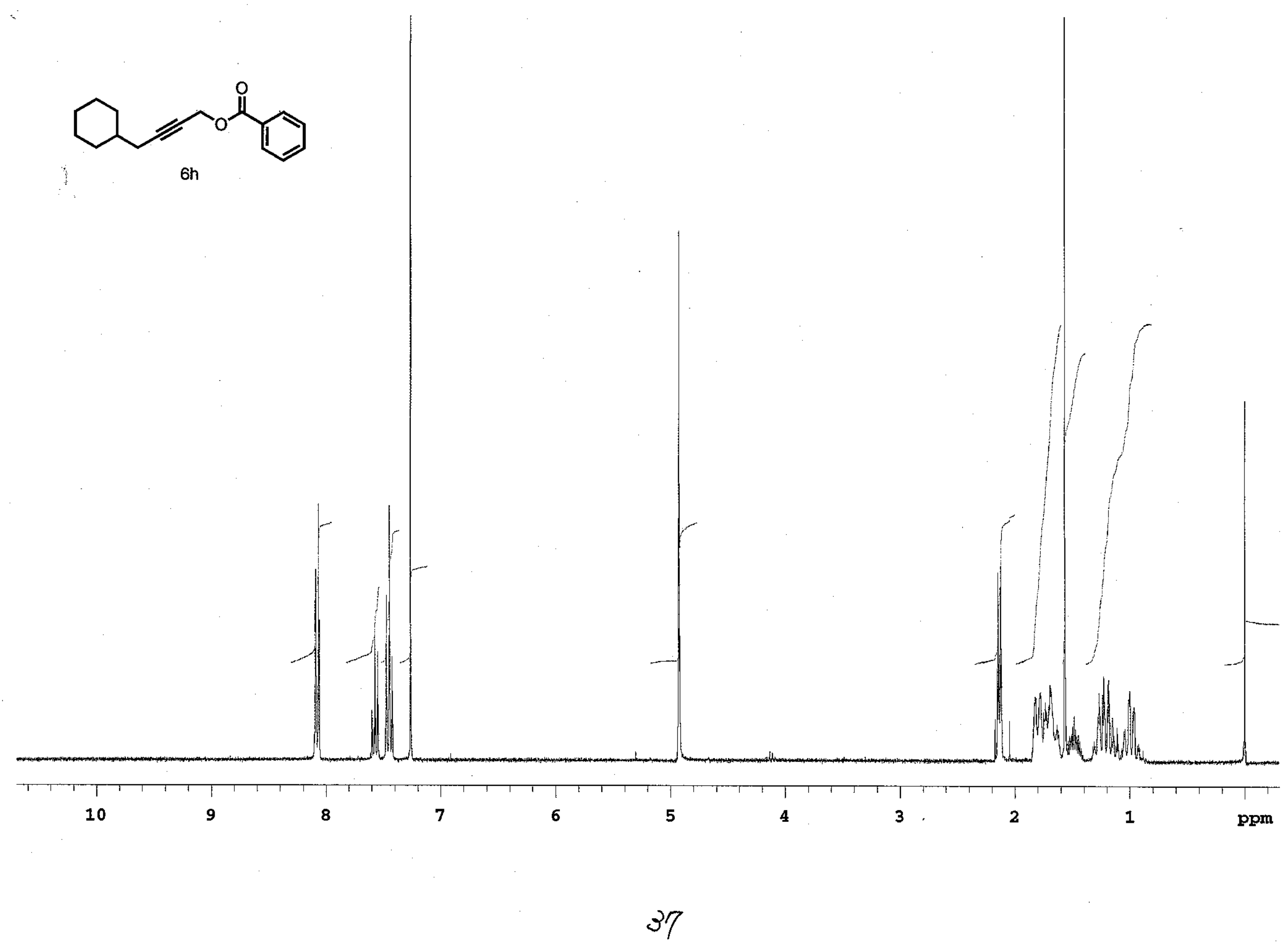




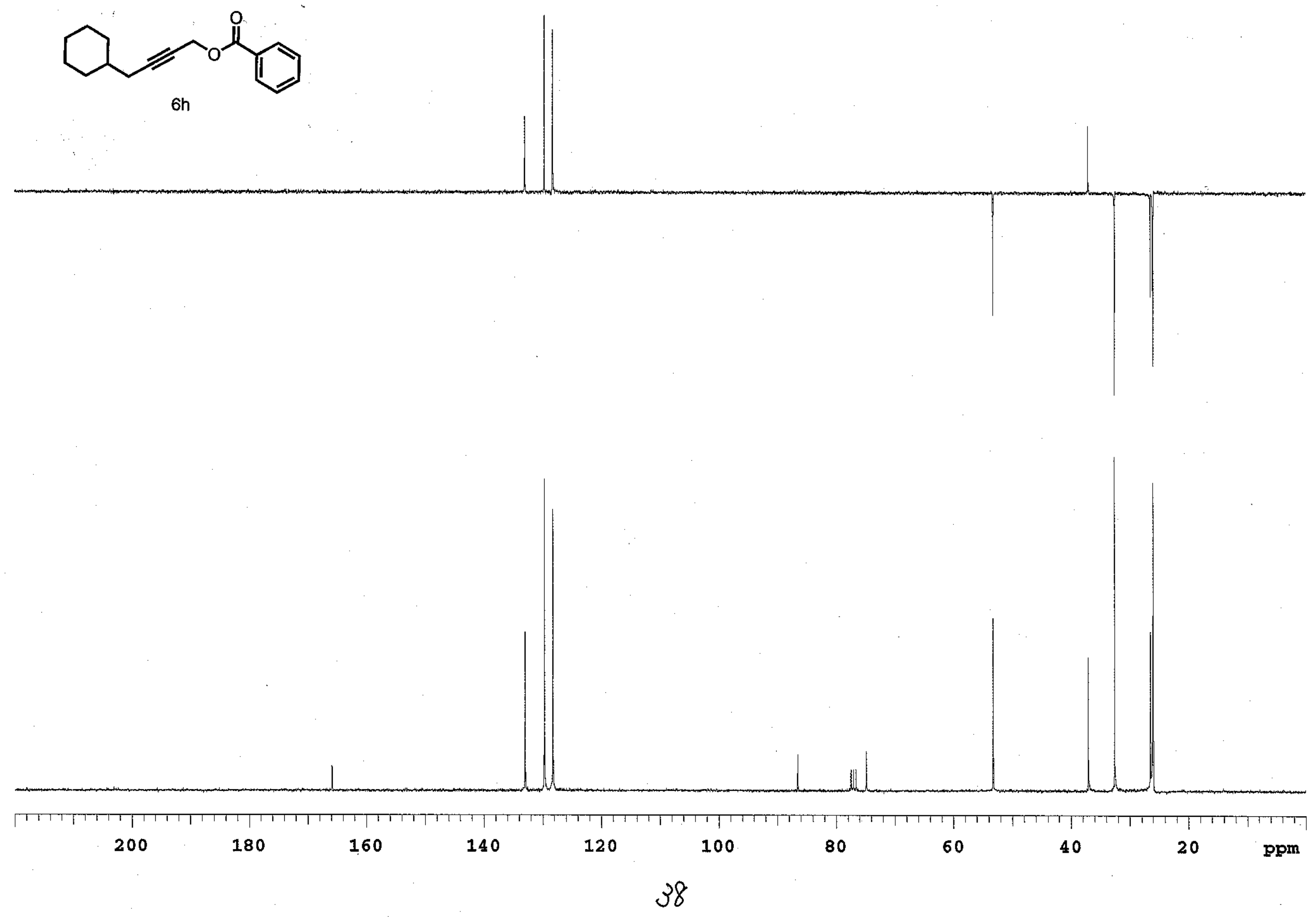




$$
=1
$$



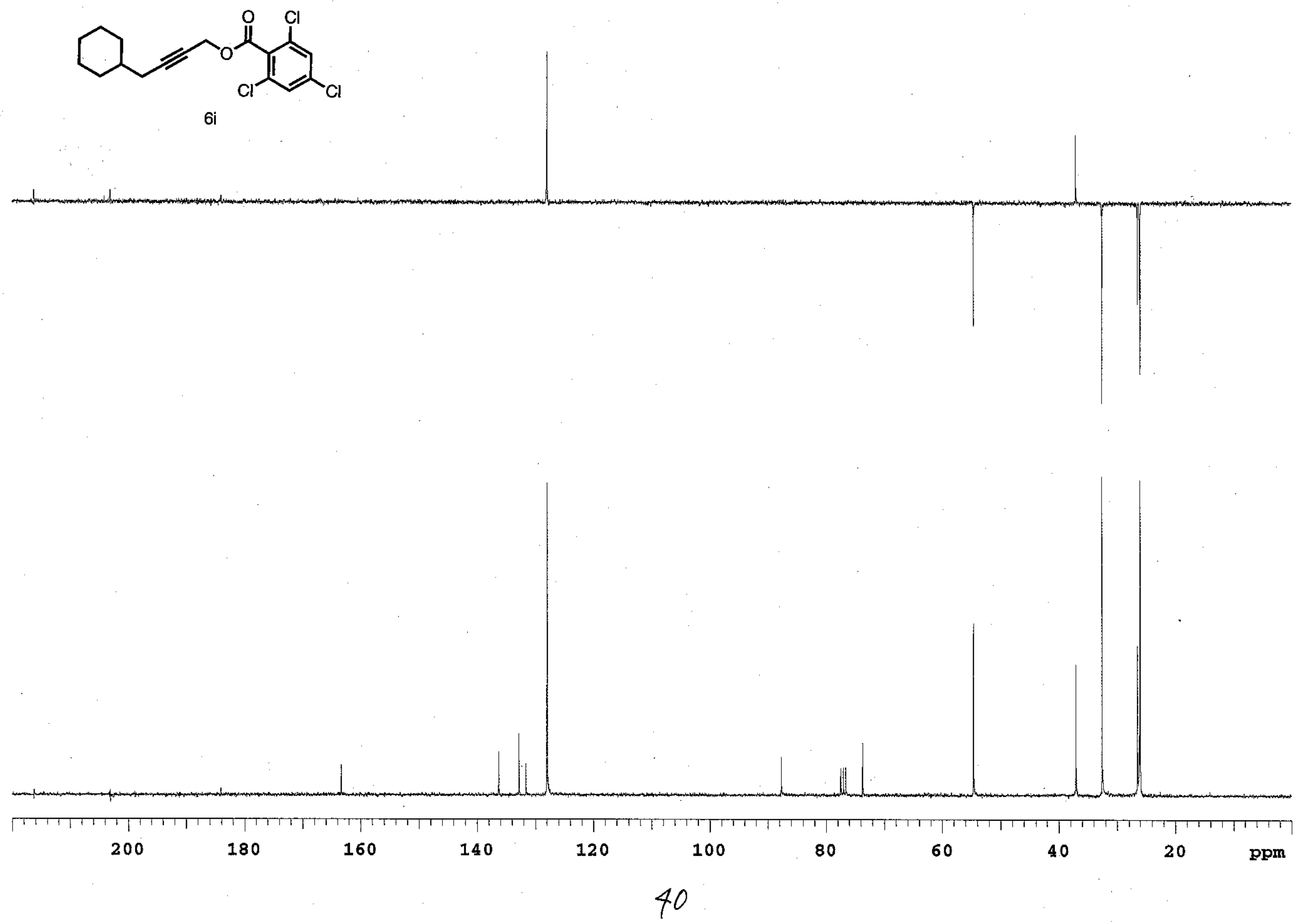


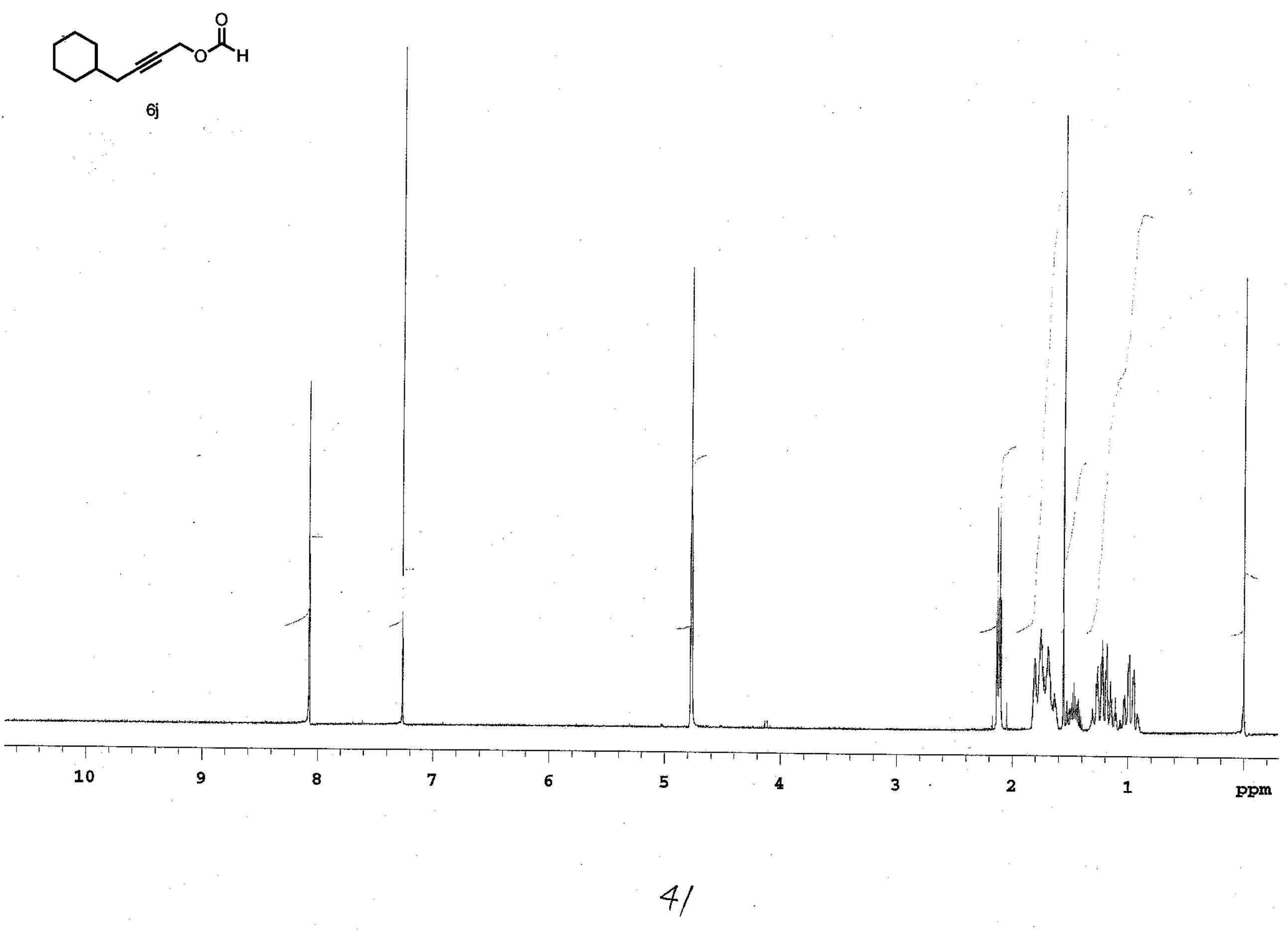




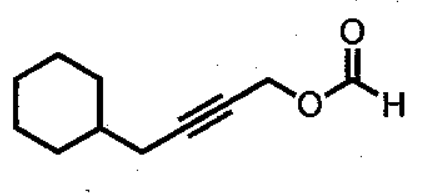

6j
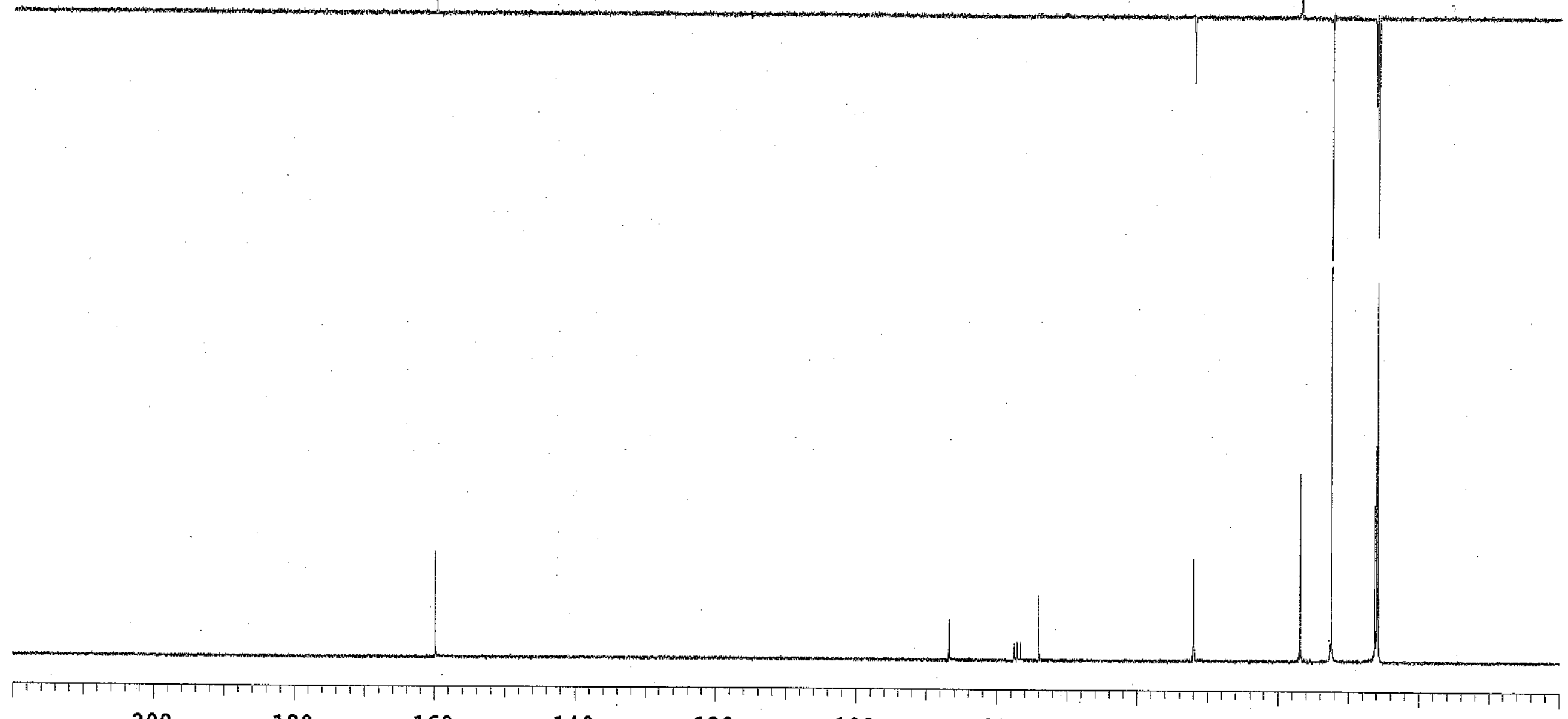


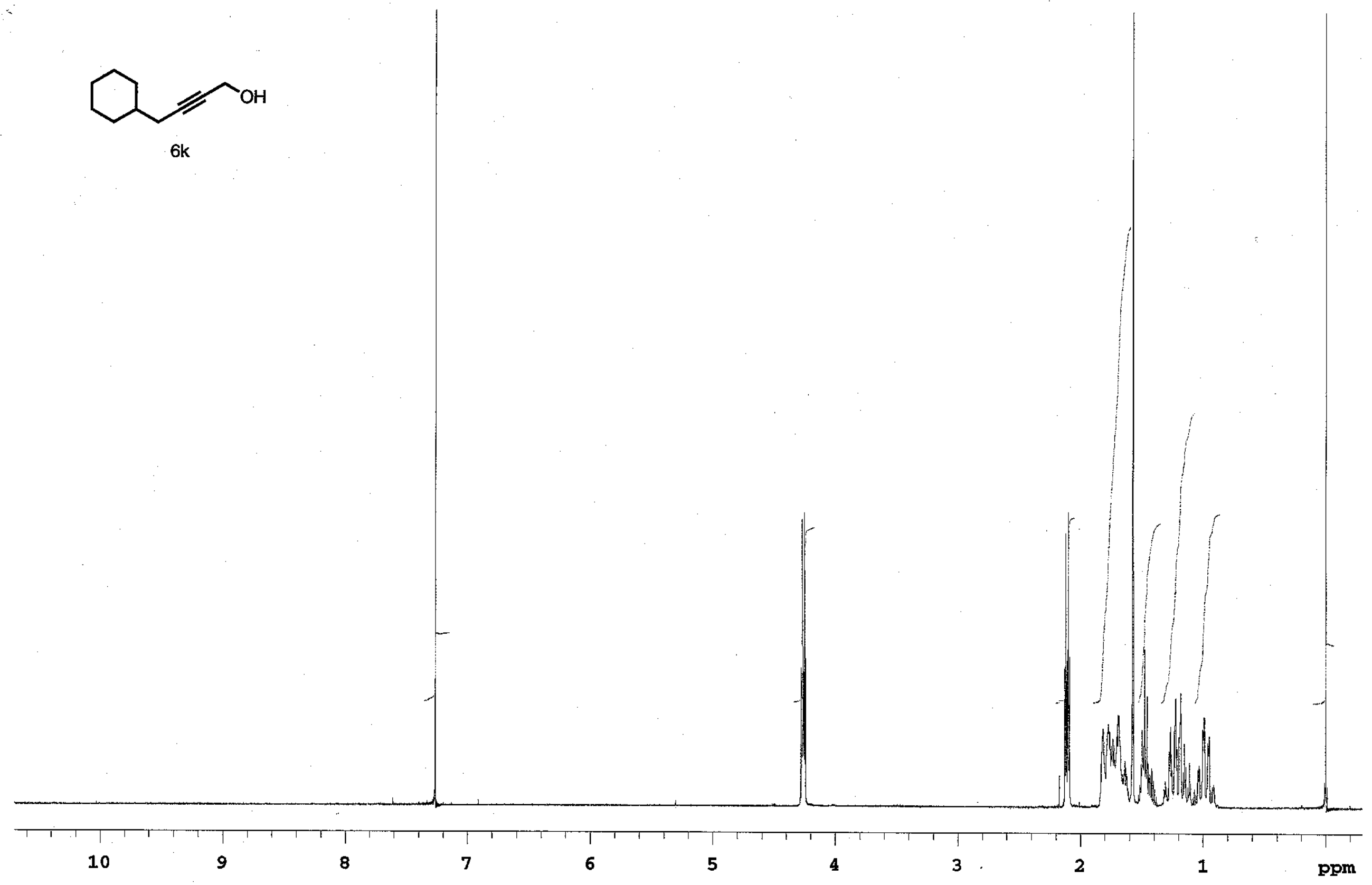




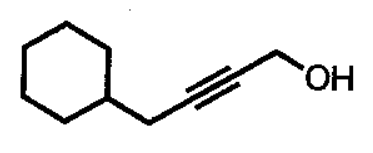

$6 k$
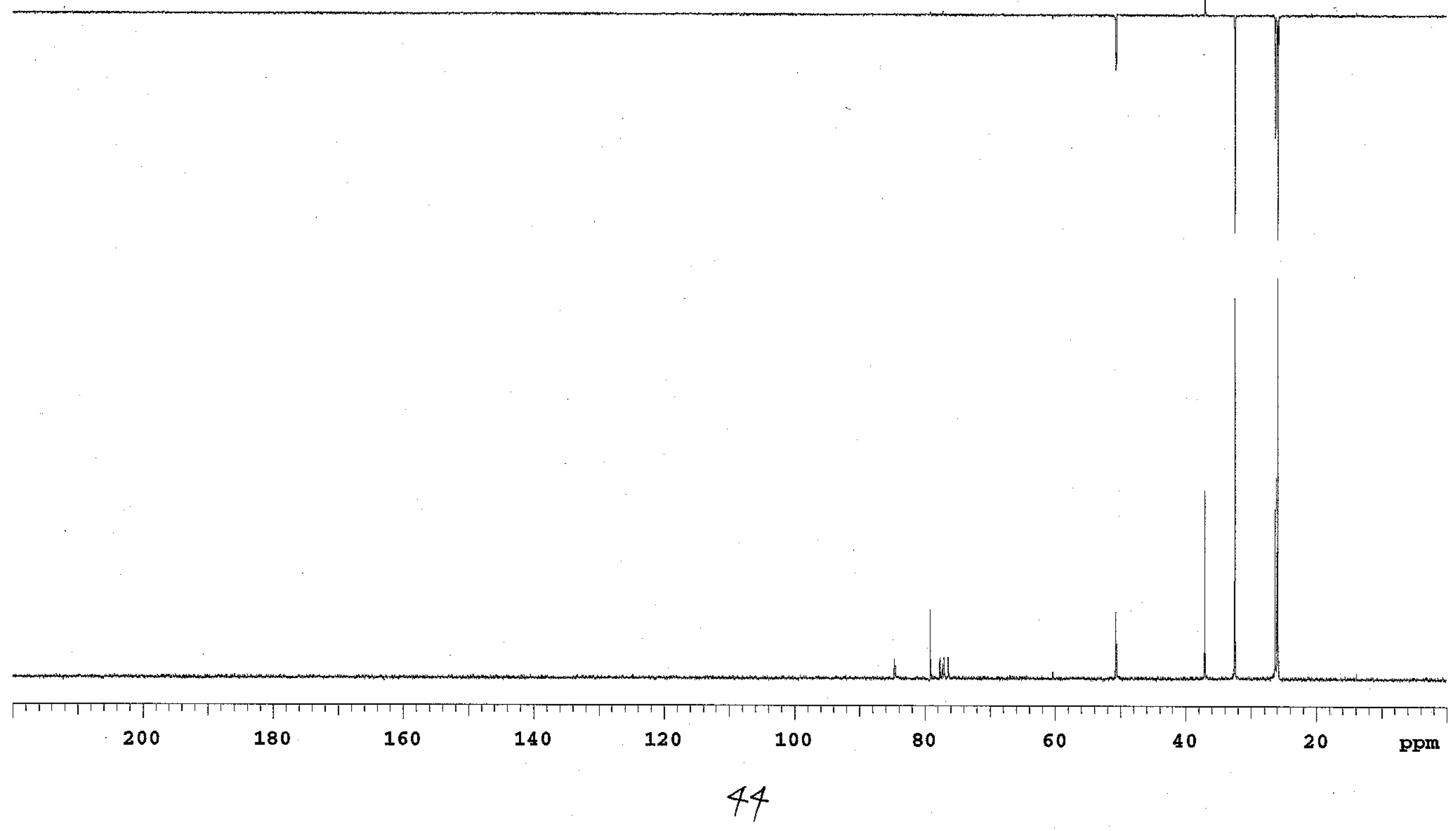


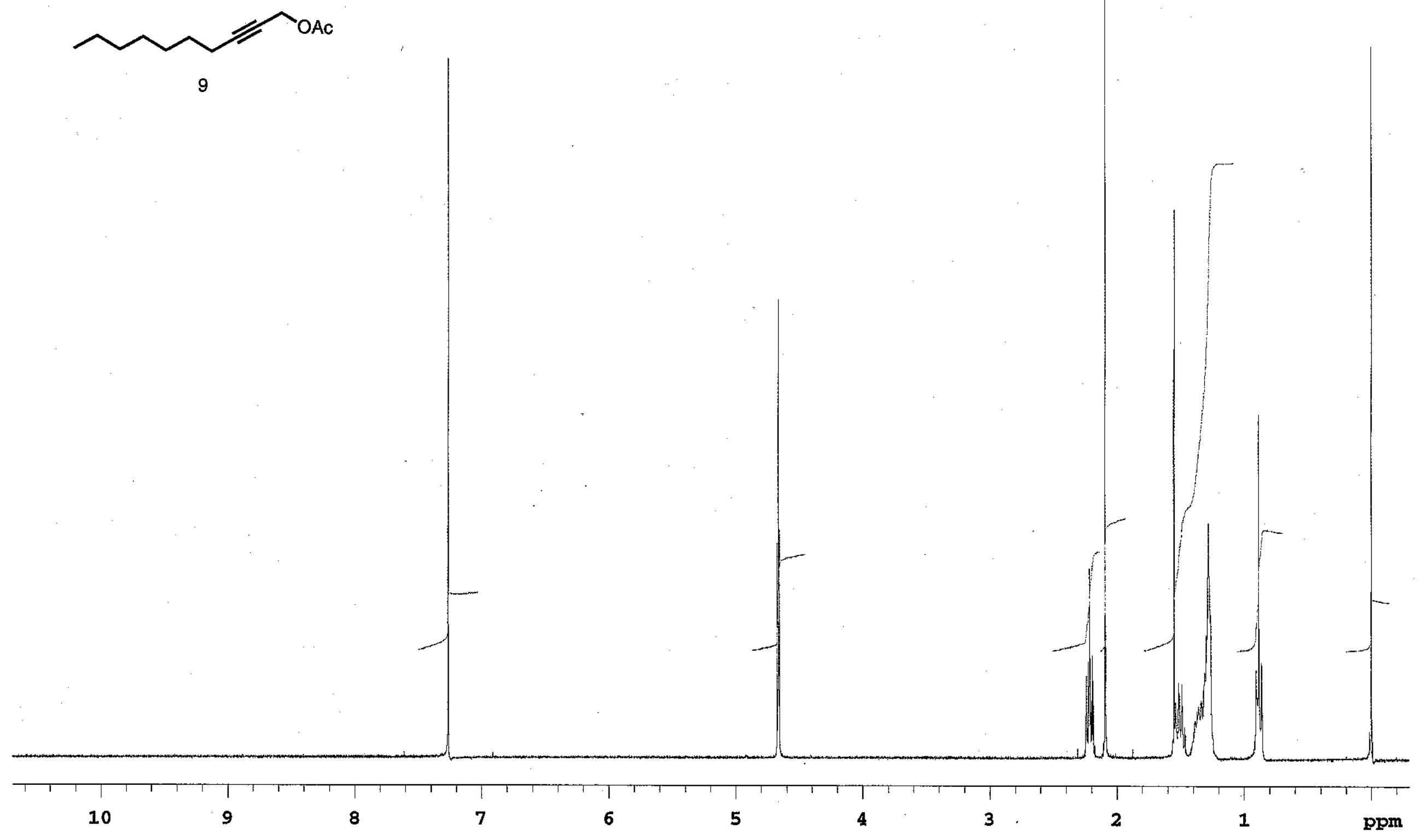




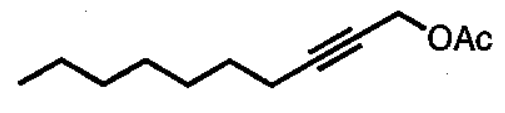

9

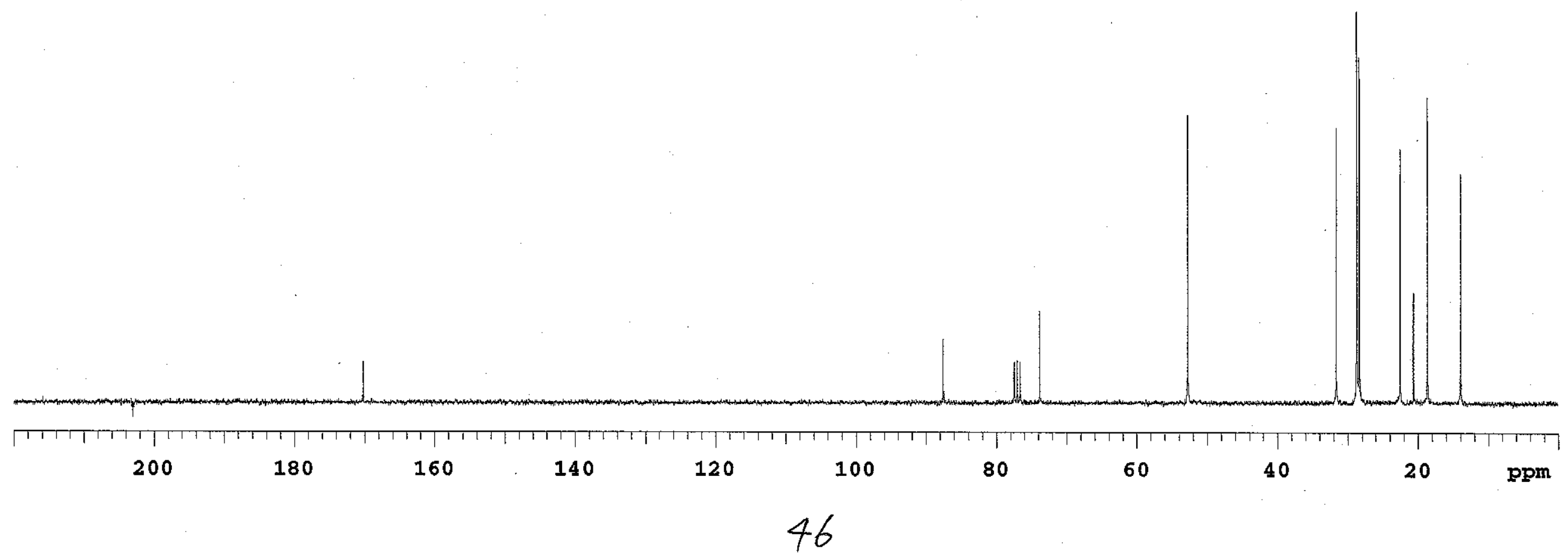


$\sim^{\circ}$

10

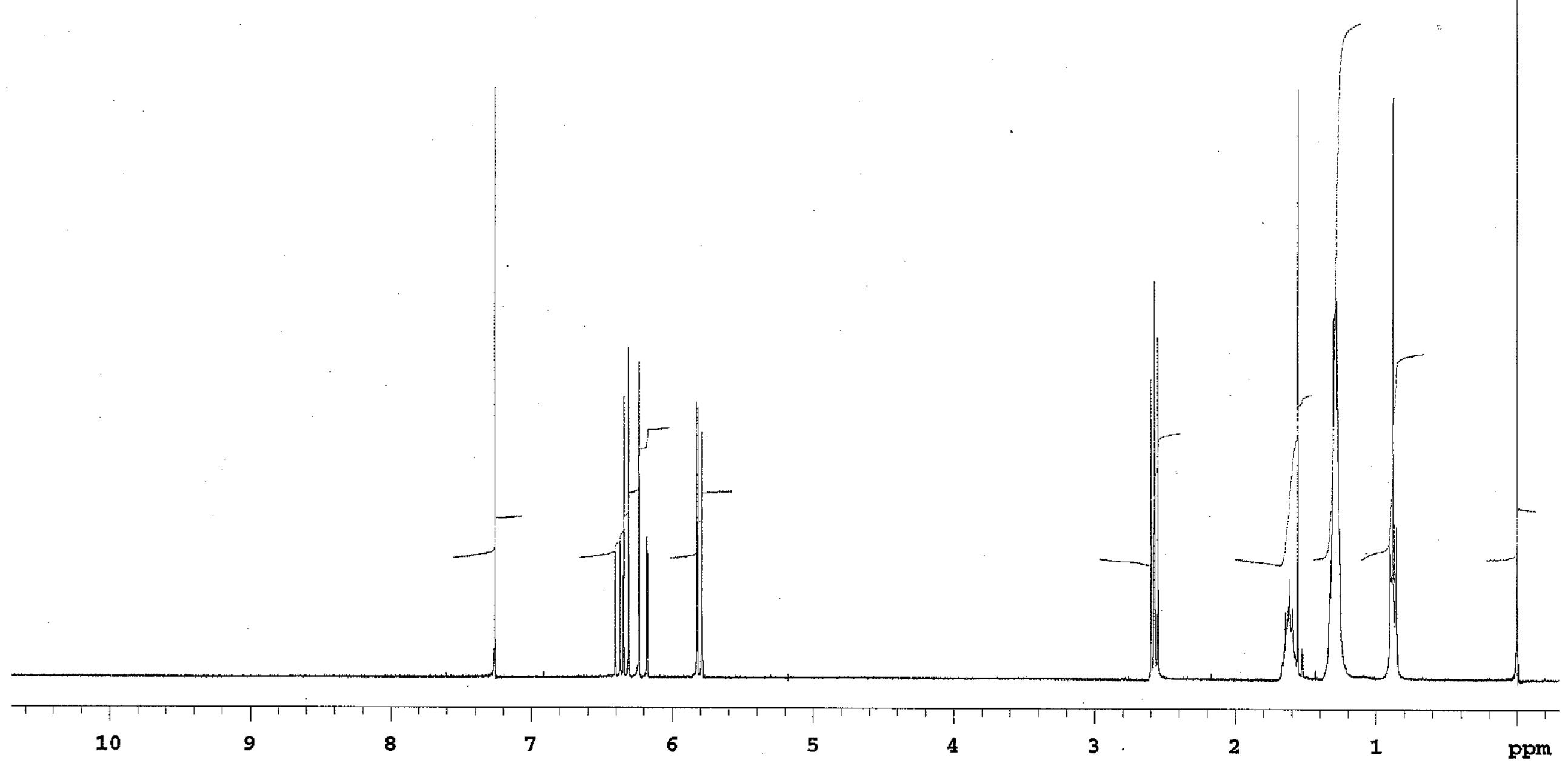




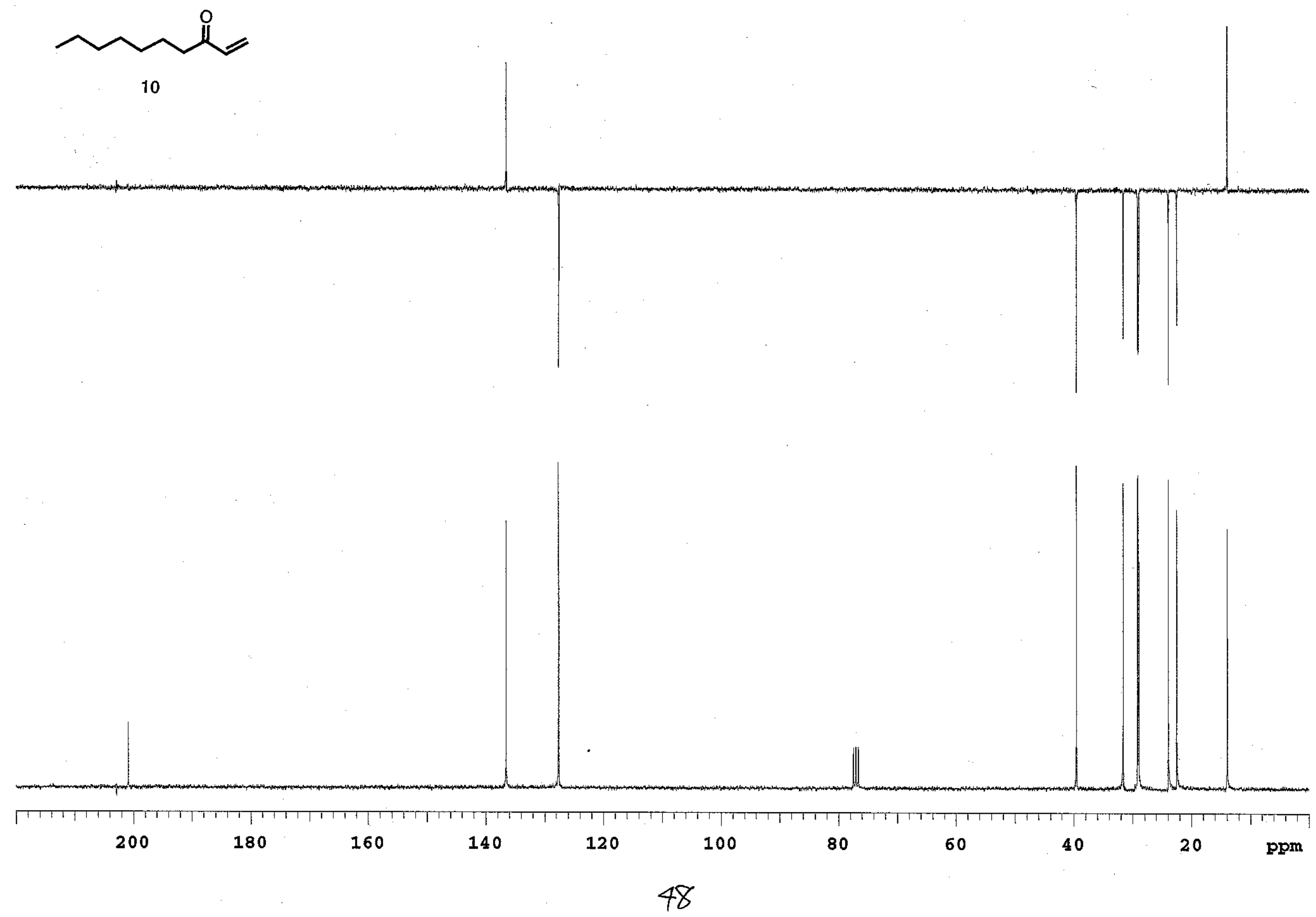



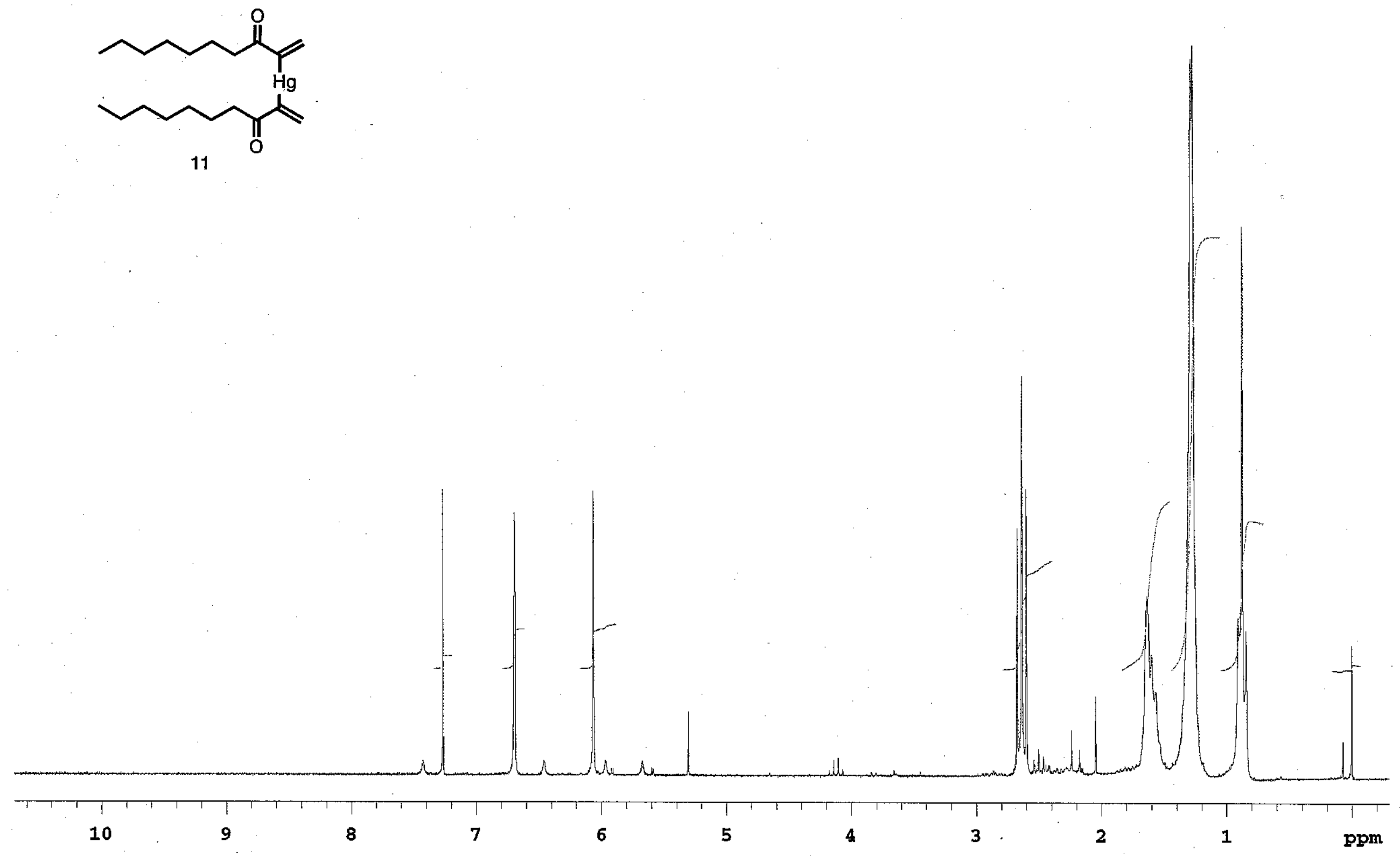


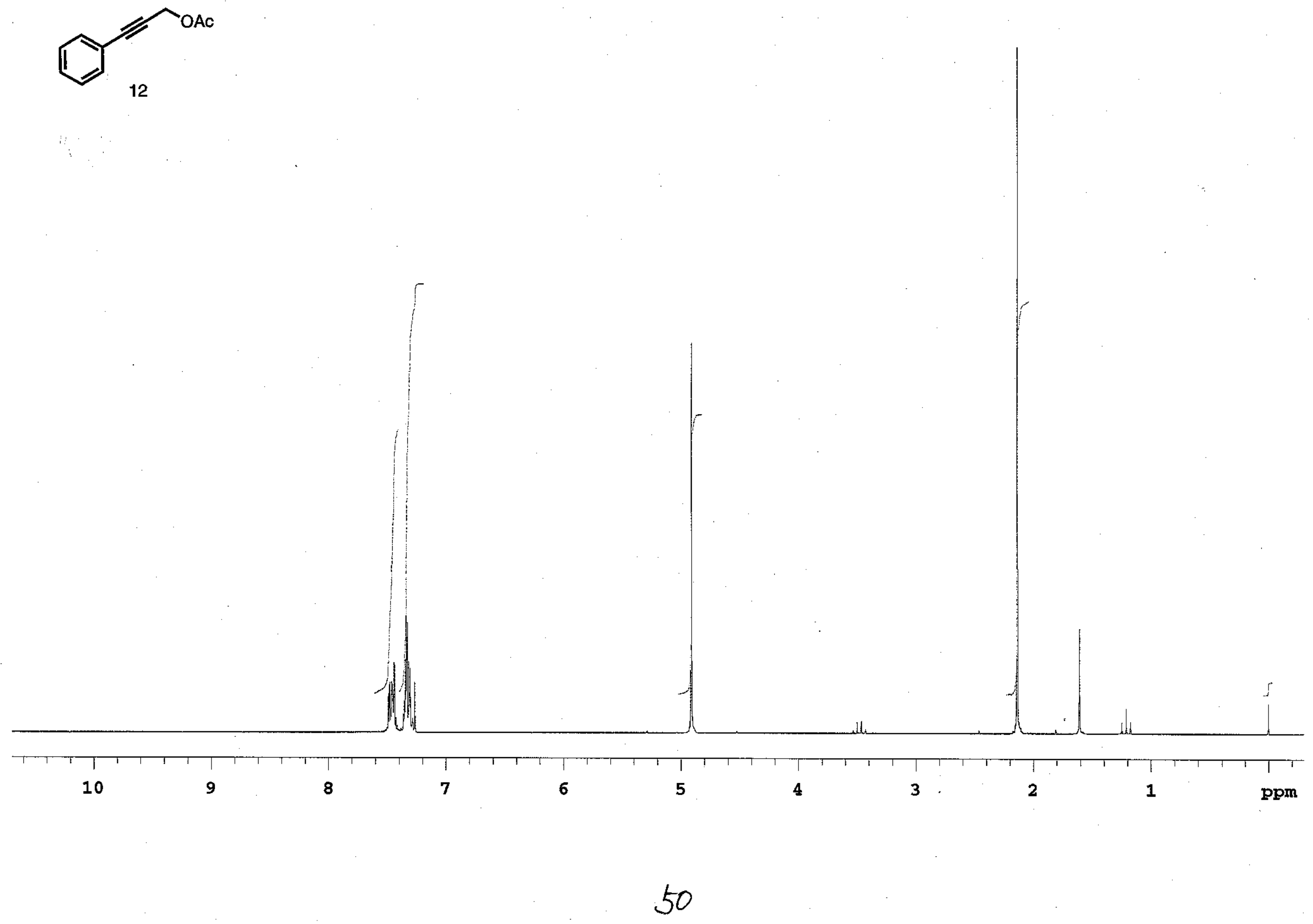




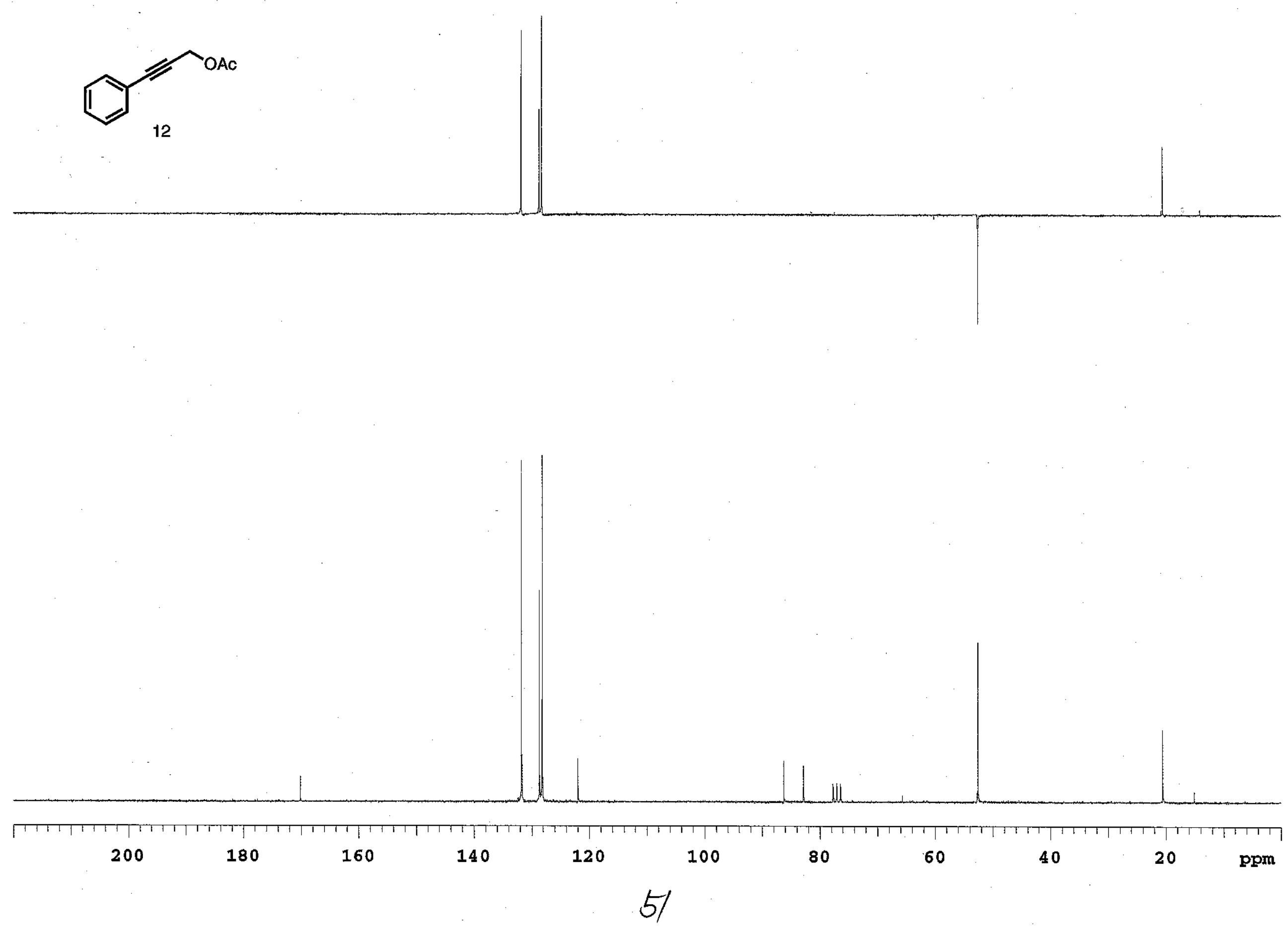




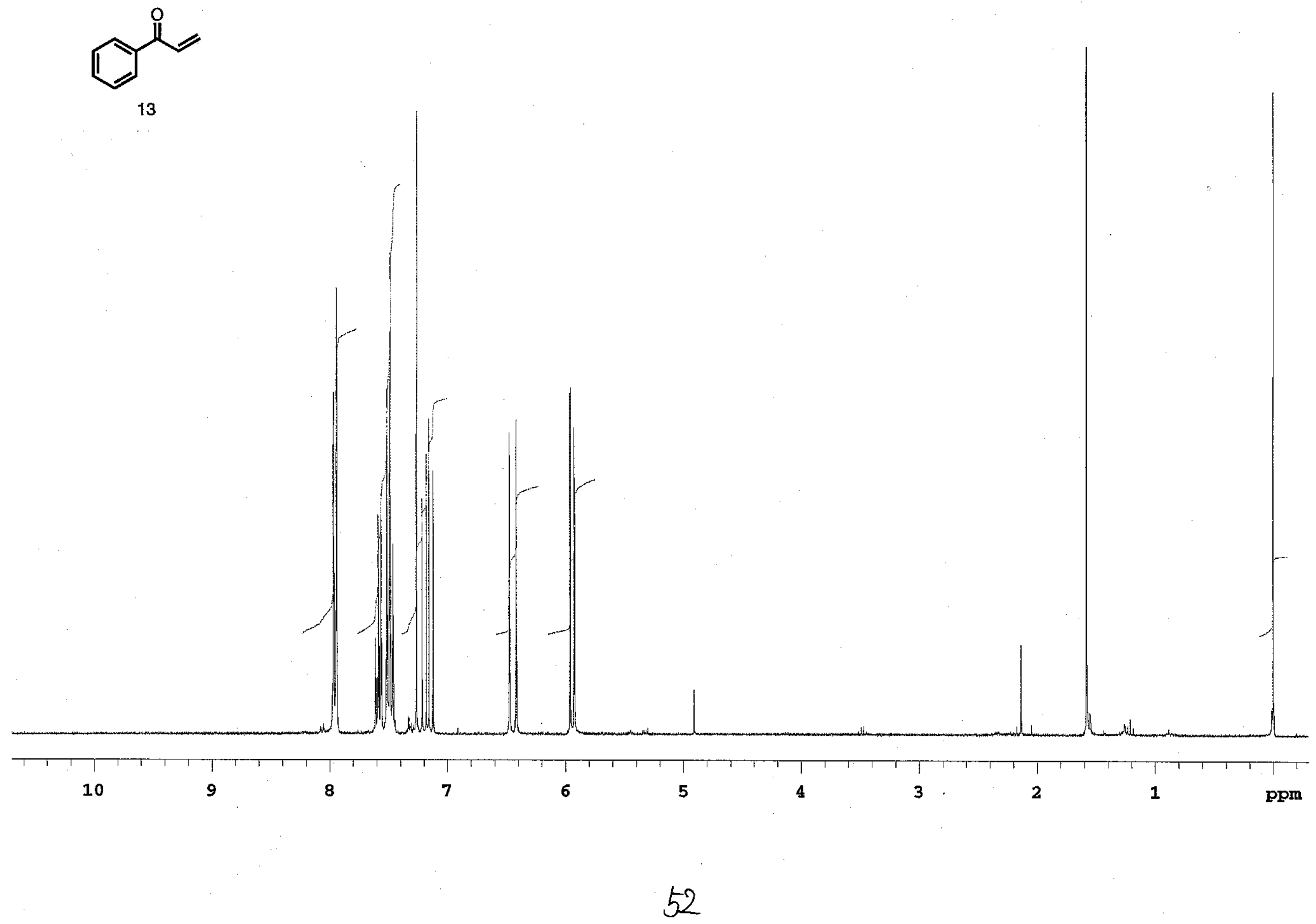




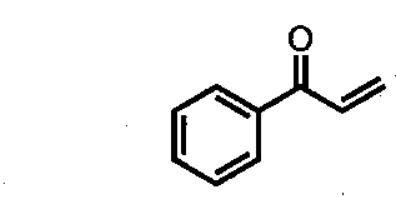

13

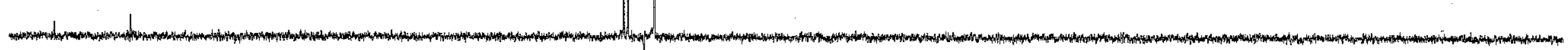

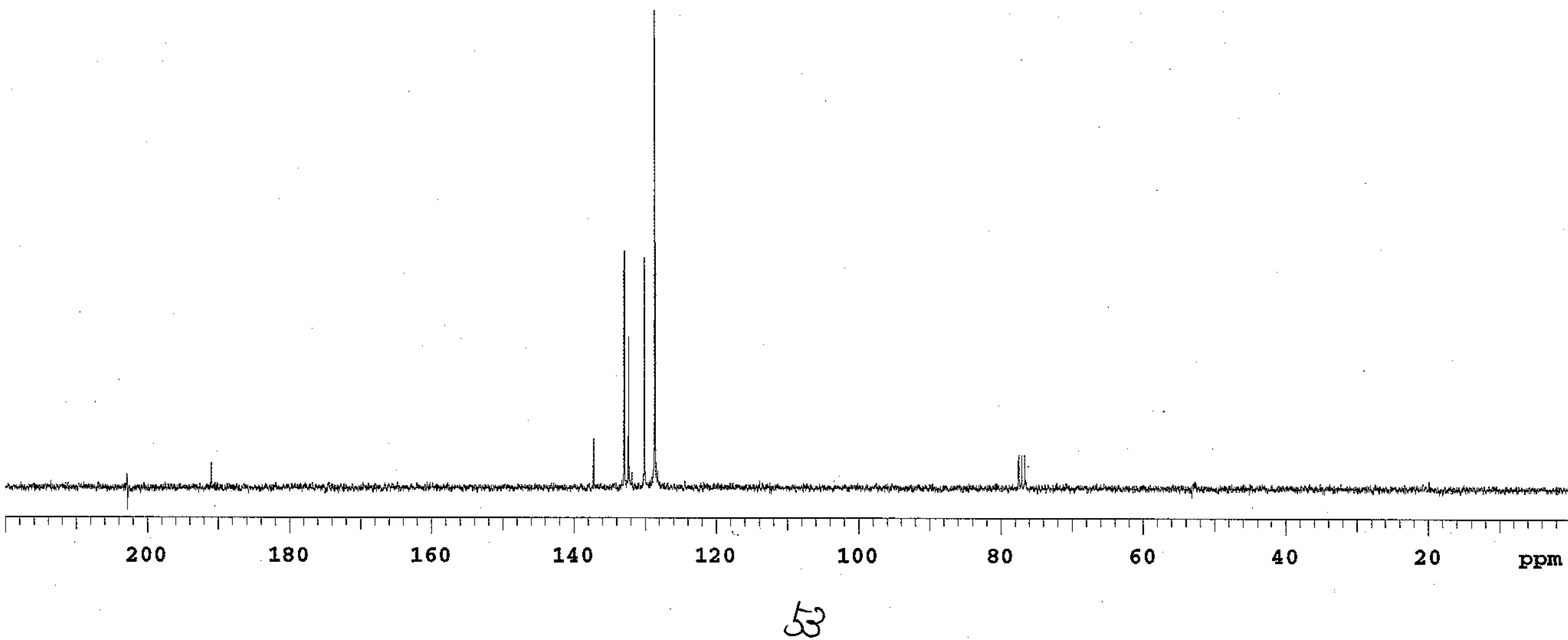




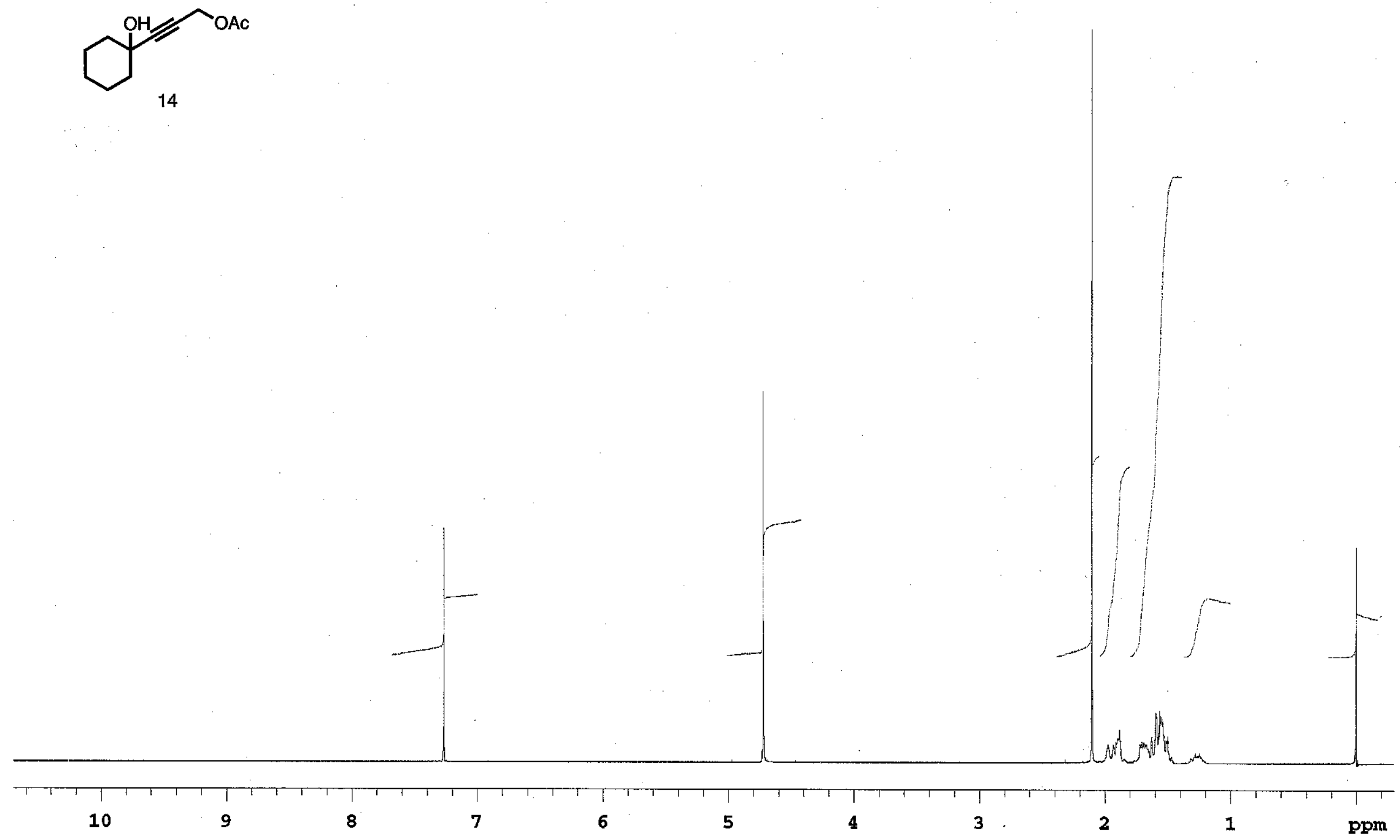

54 


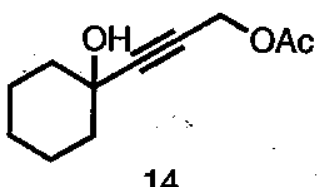

14

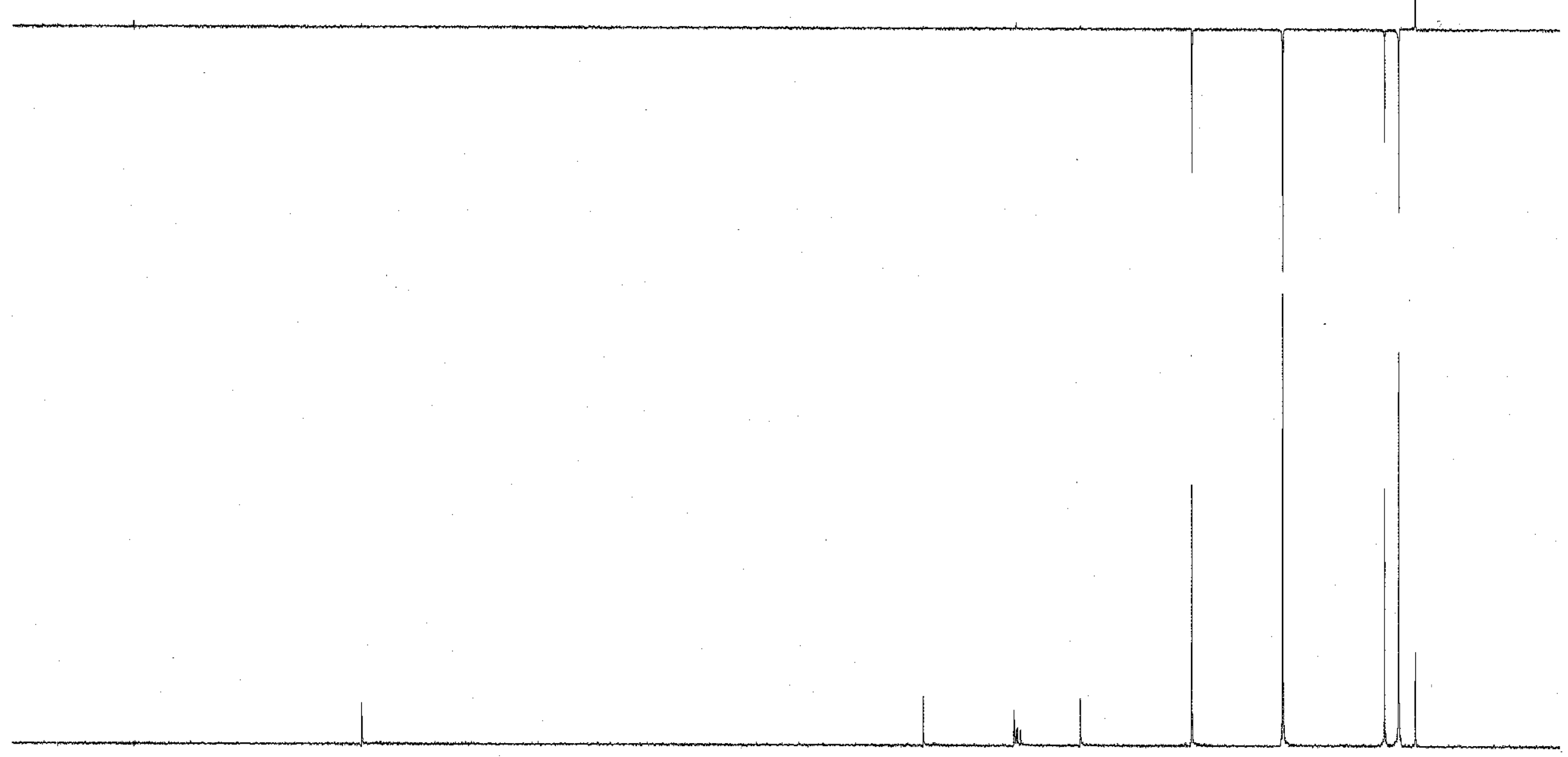

200

180

160

140

120

100

80

60

40

20

ppm 
oro

15

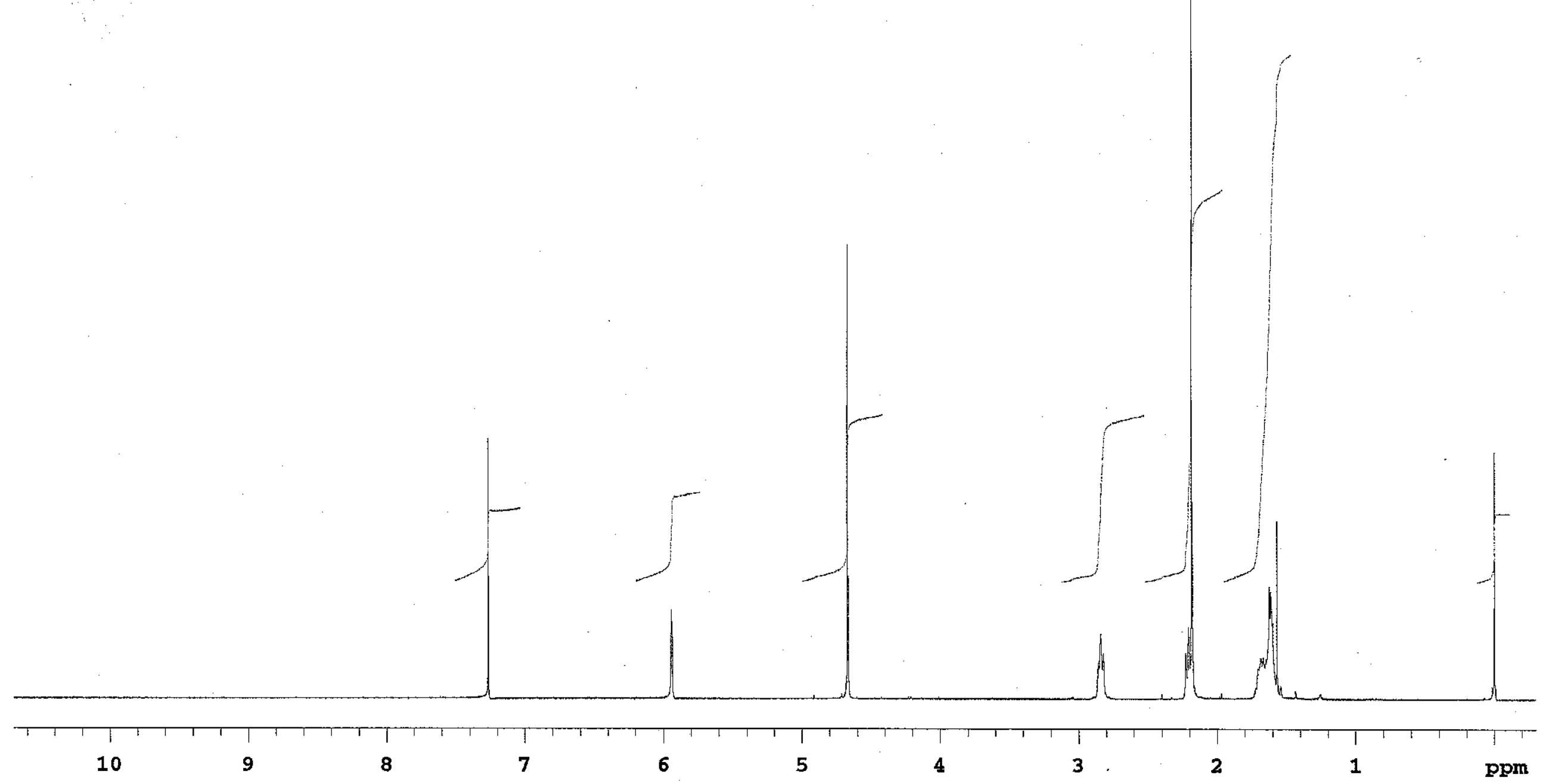


$\prod_{O A C}$

15

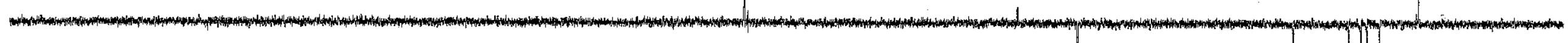
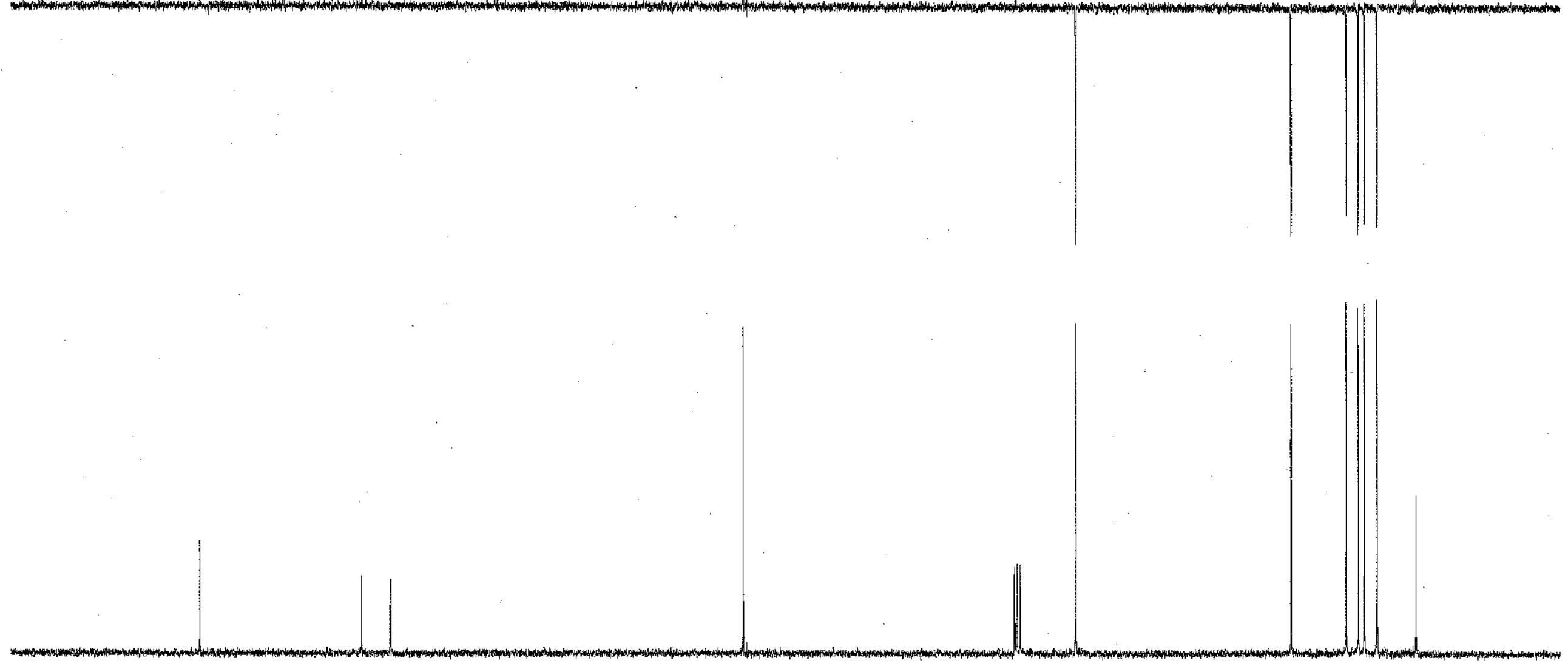

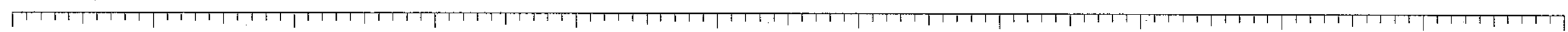



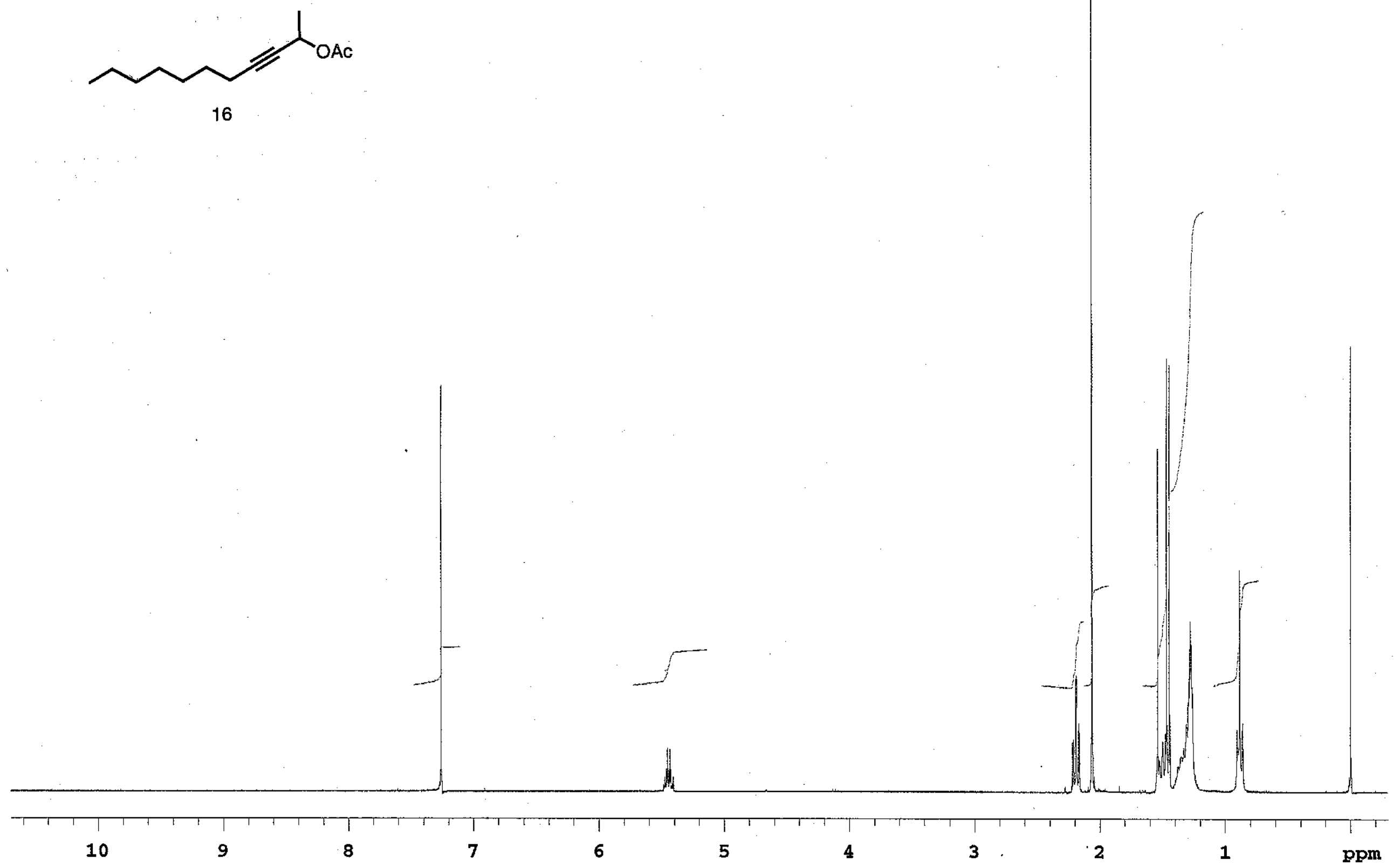


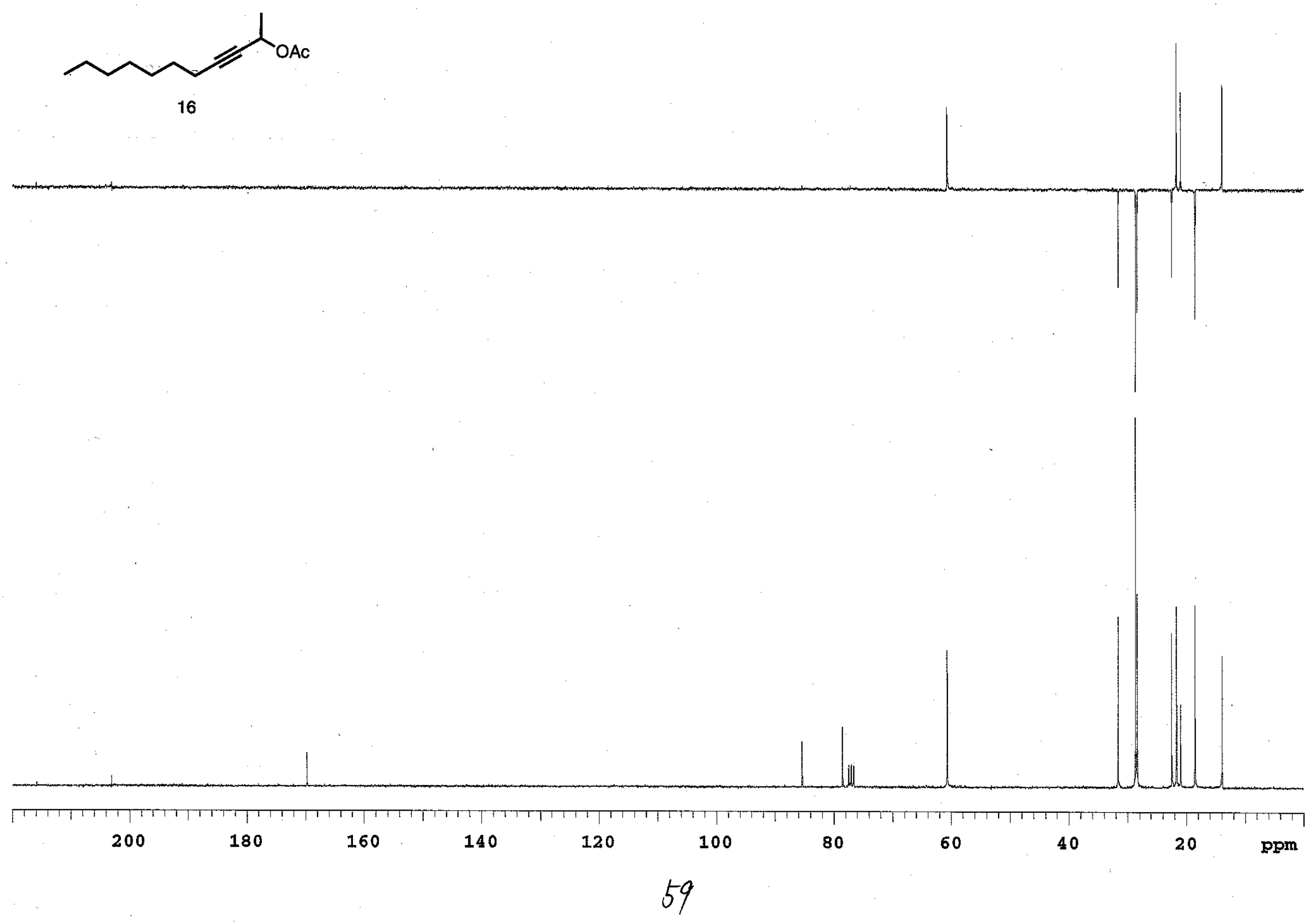



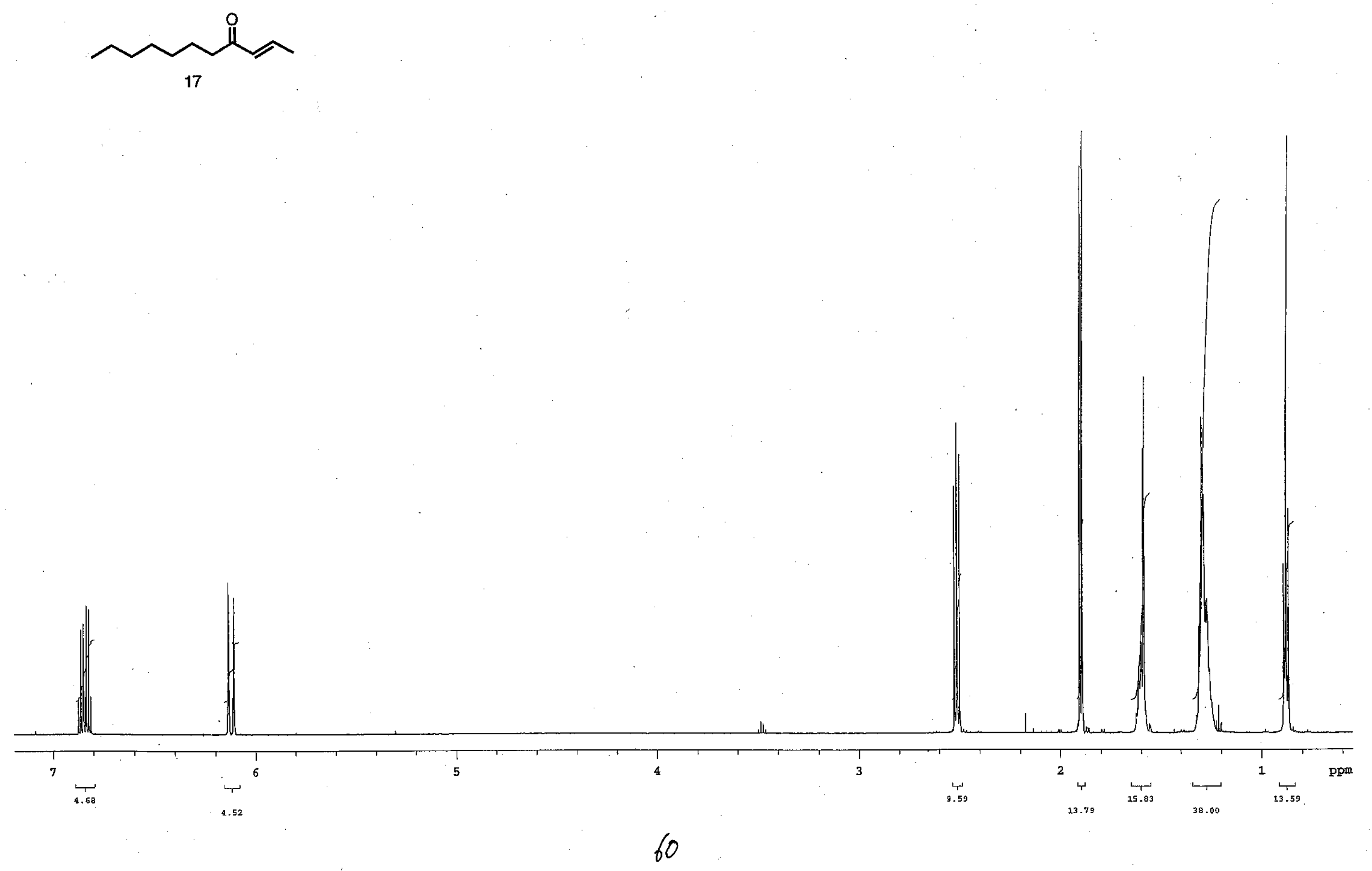

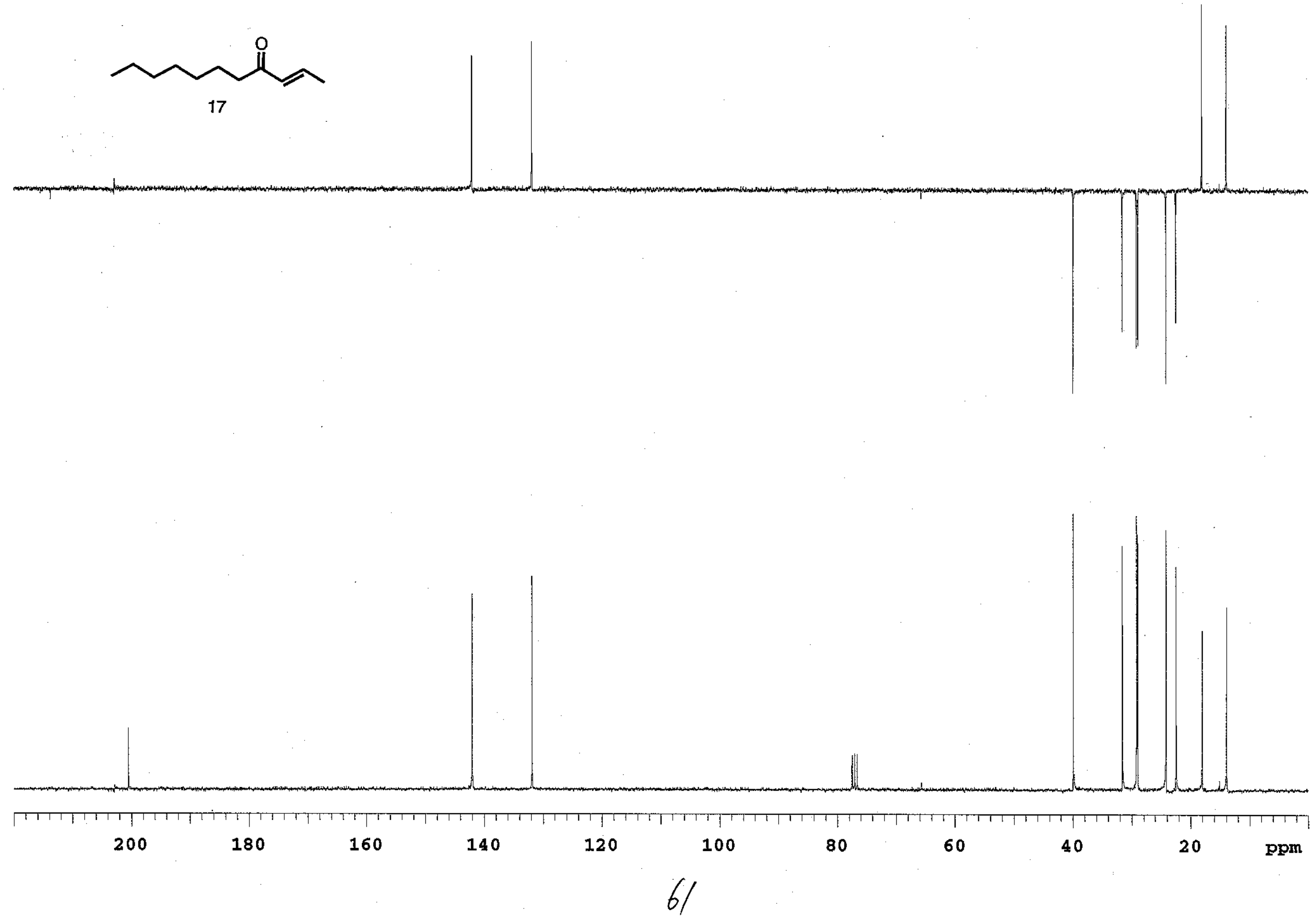


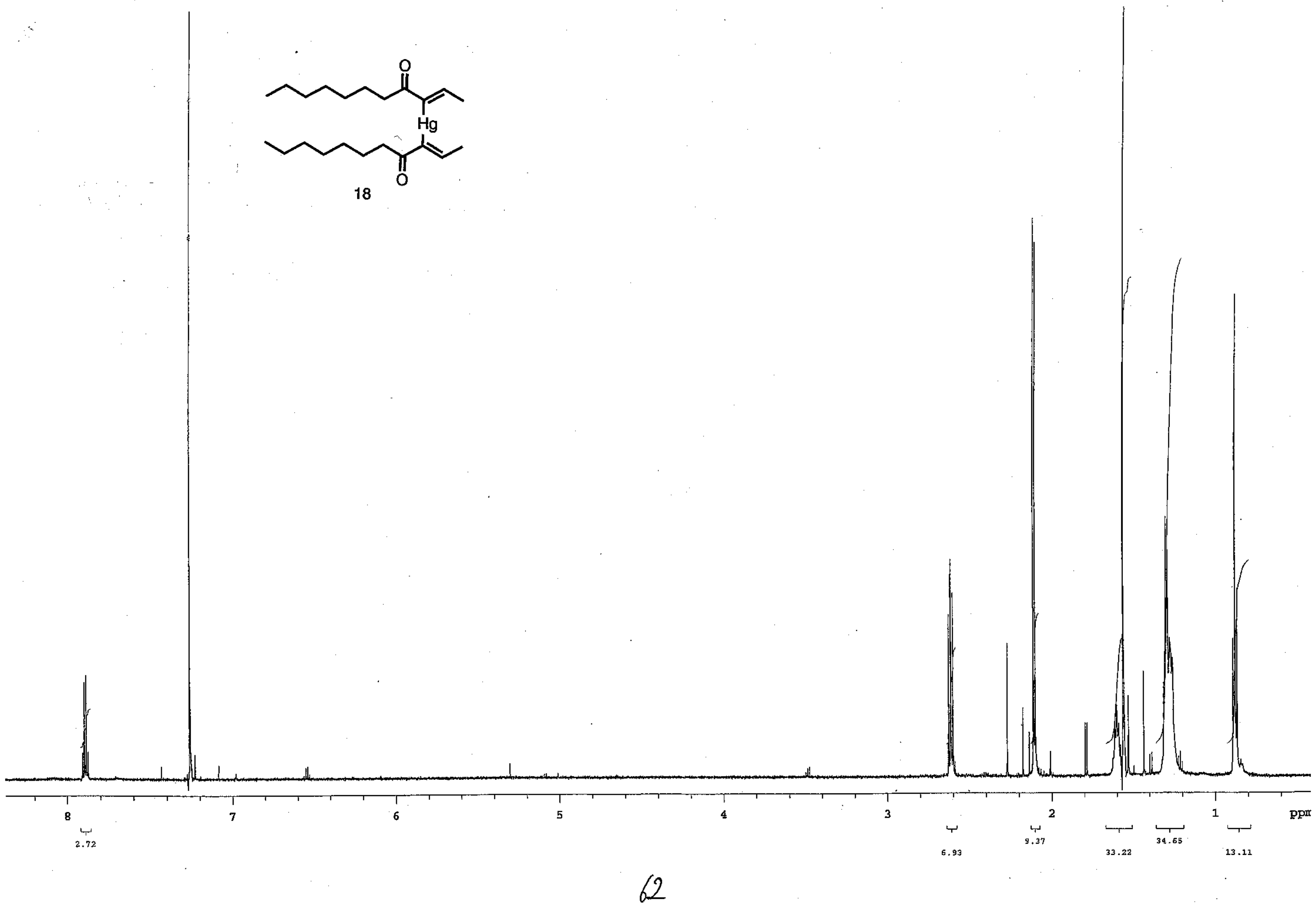




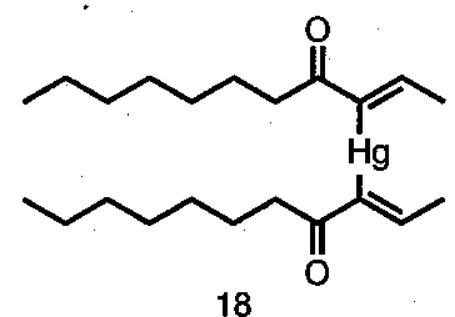

18

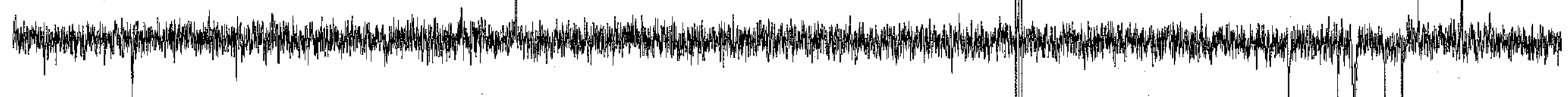

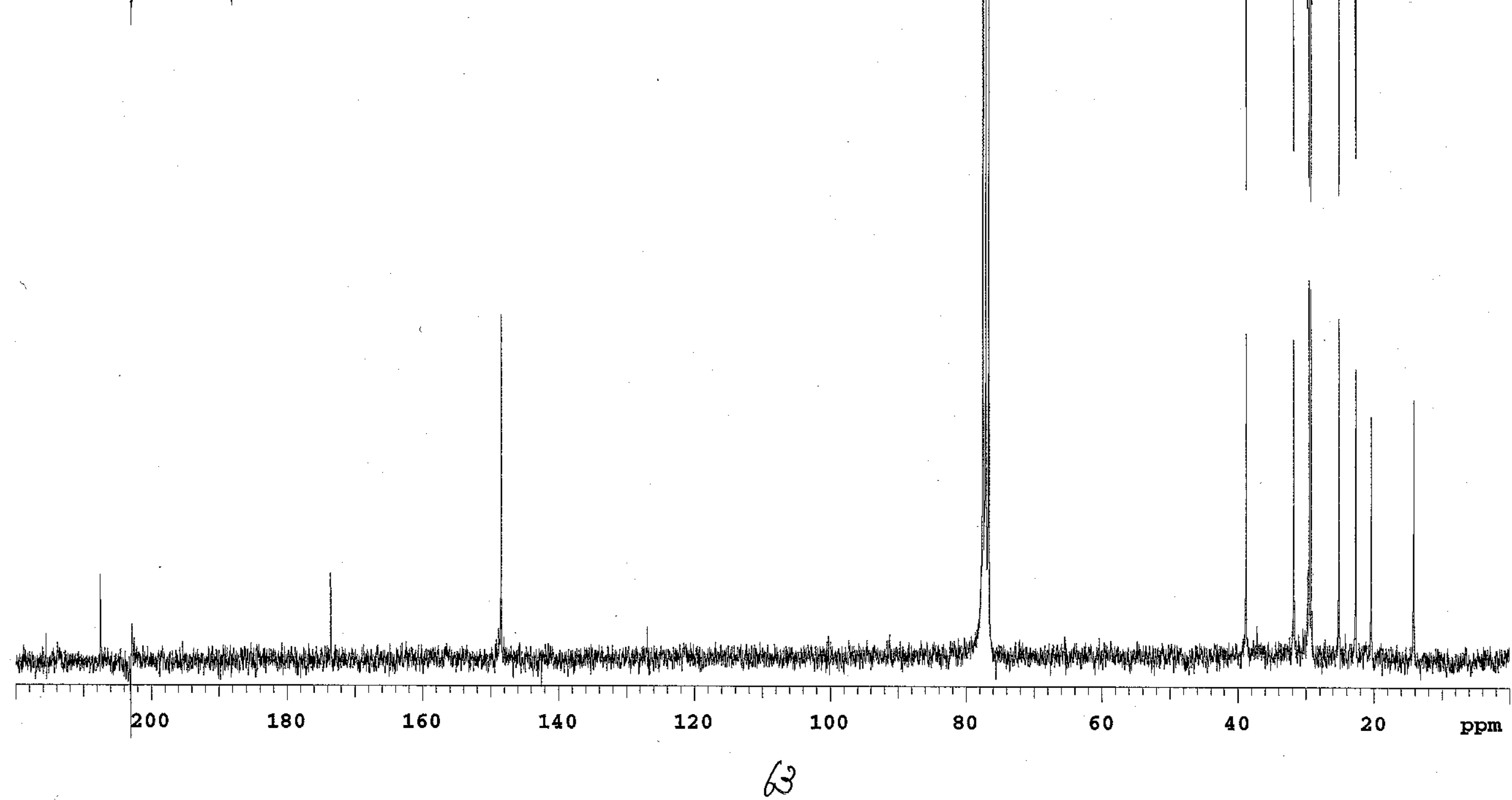



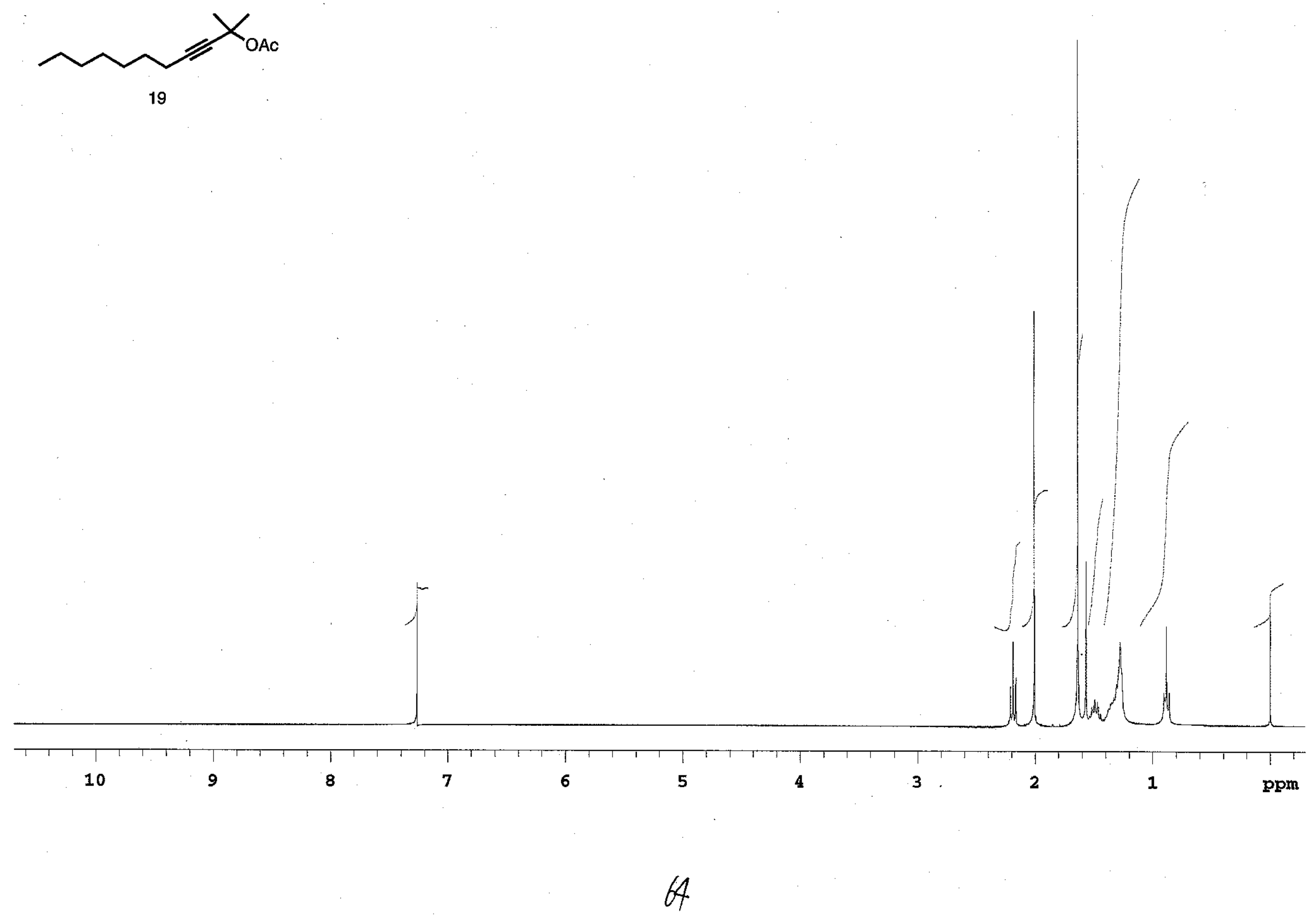


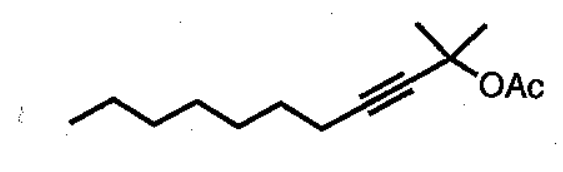

19

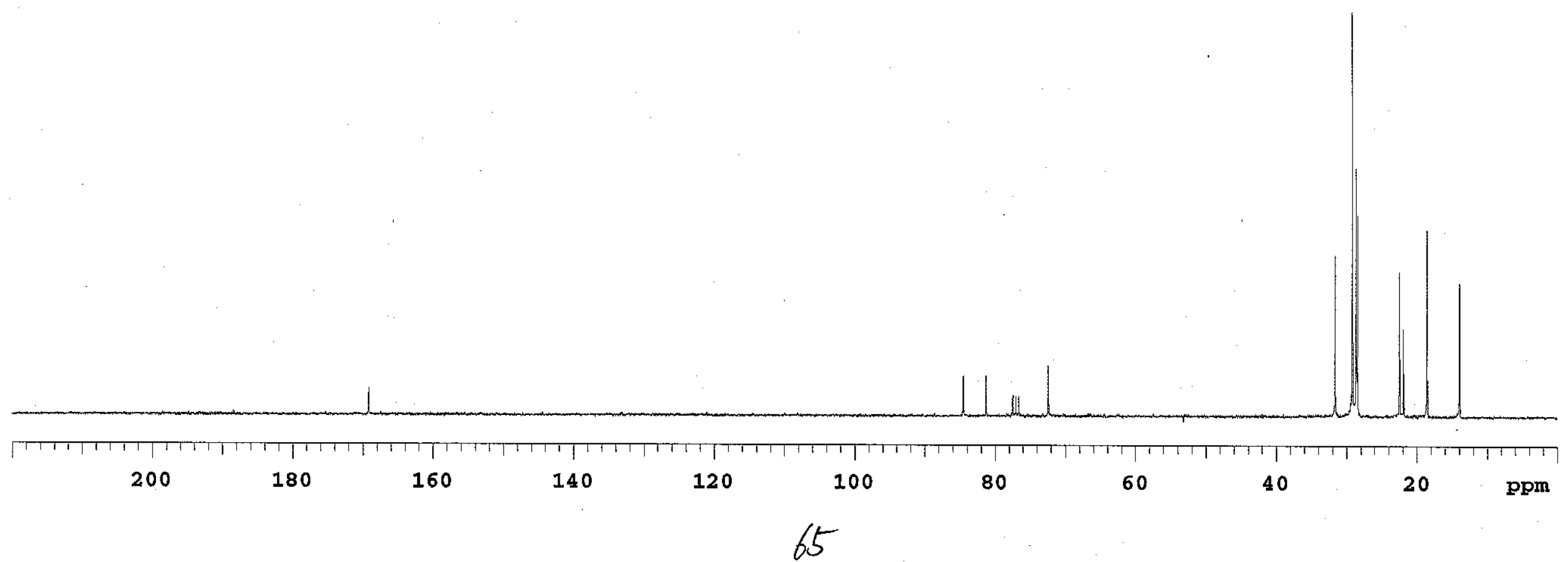




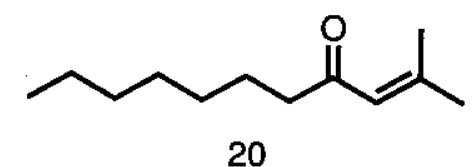

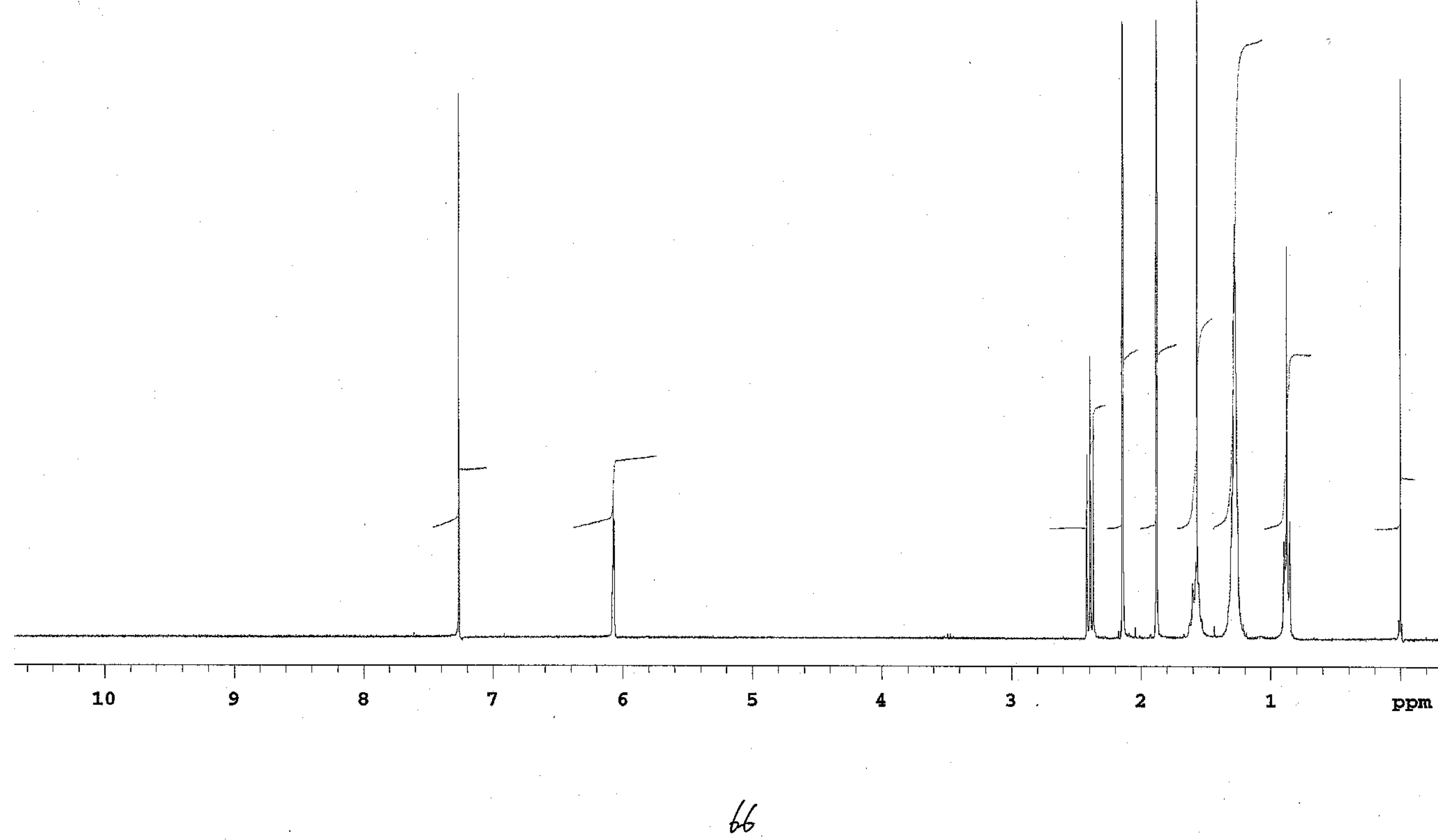




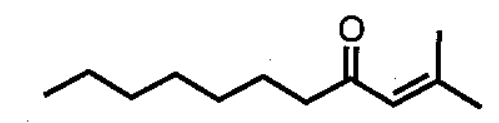

20
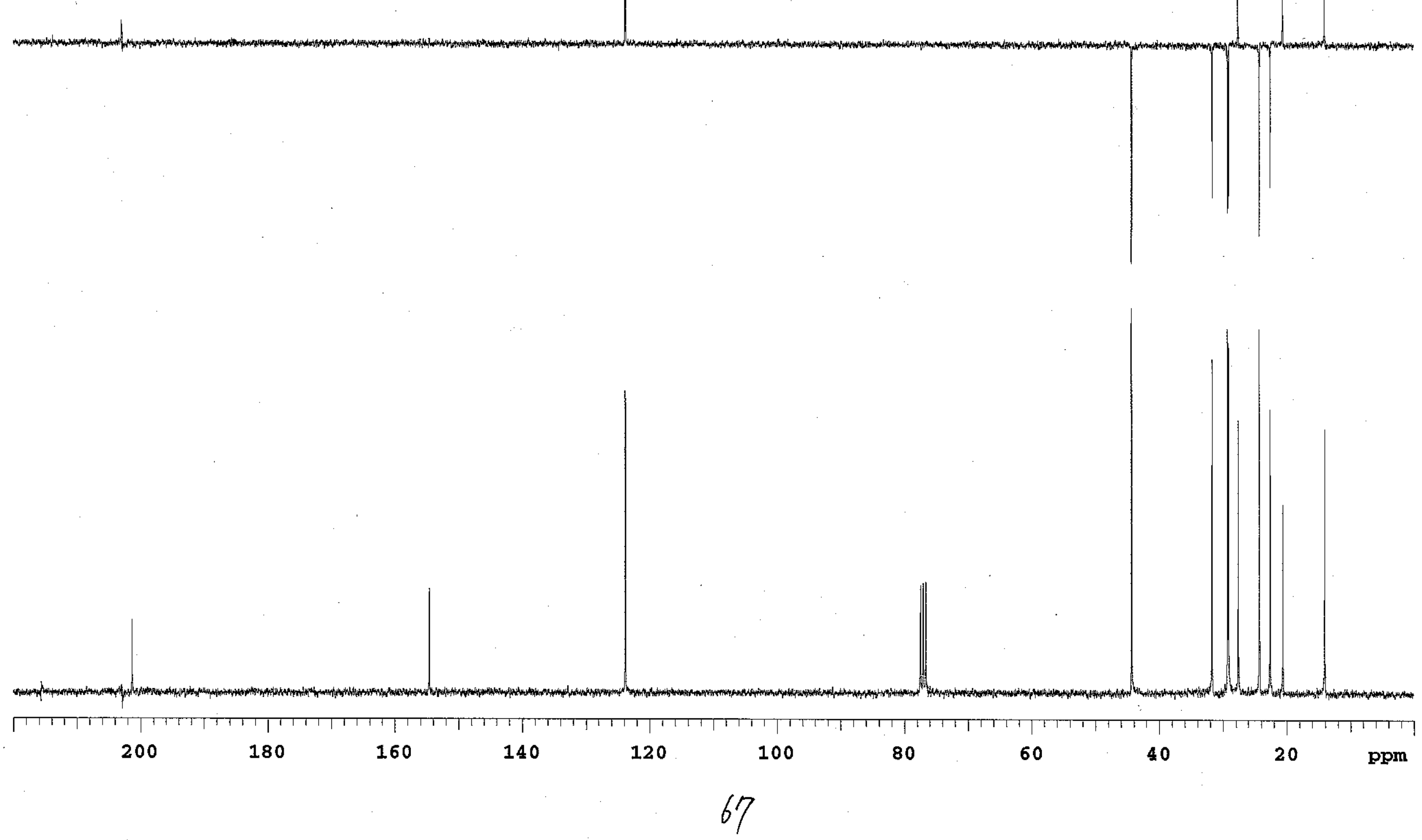


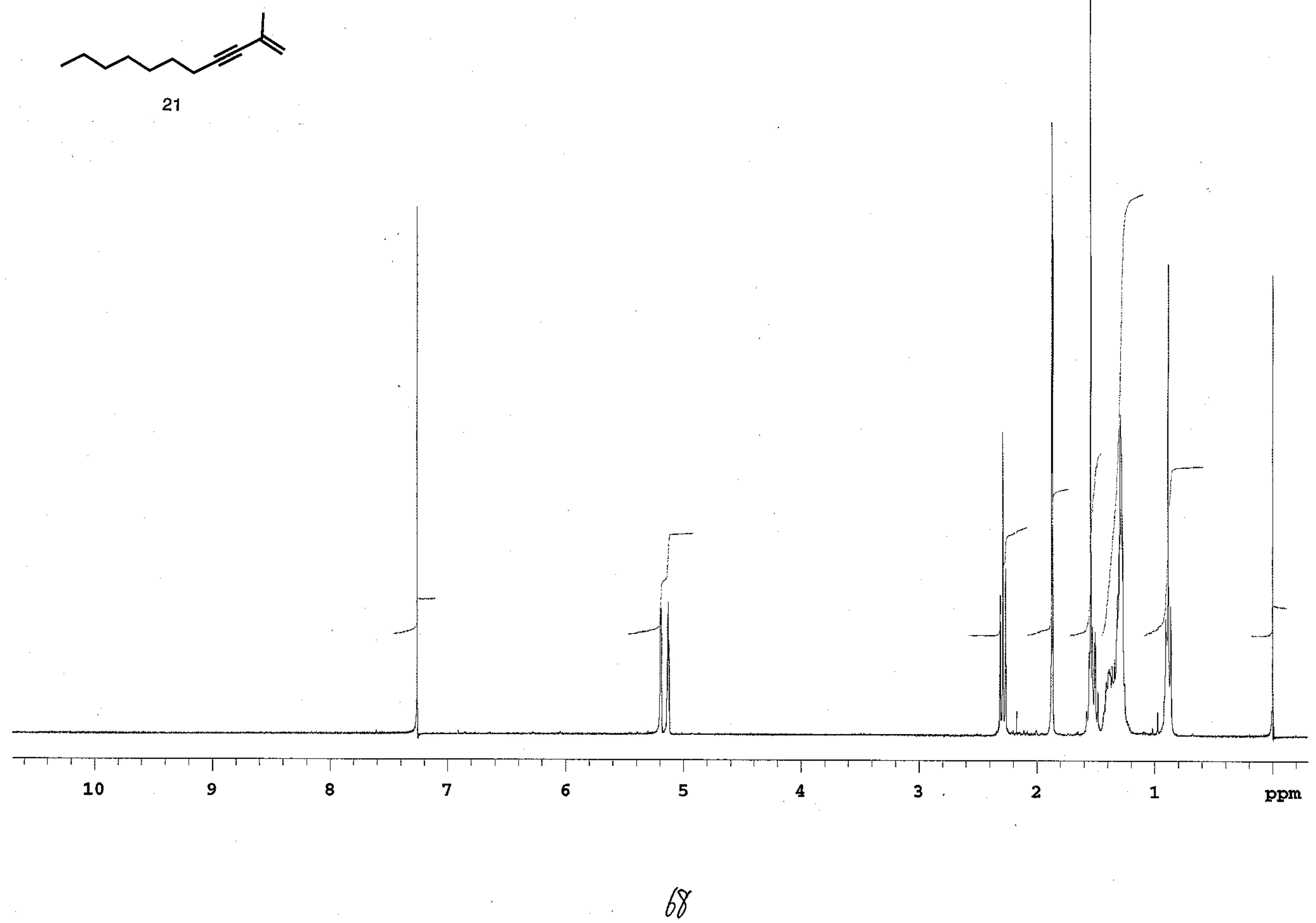



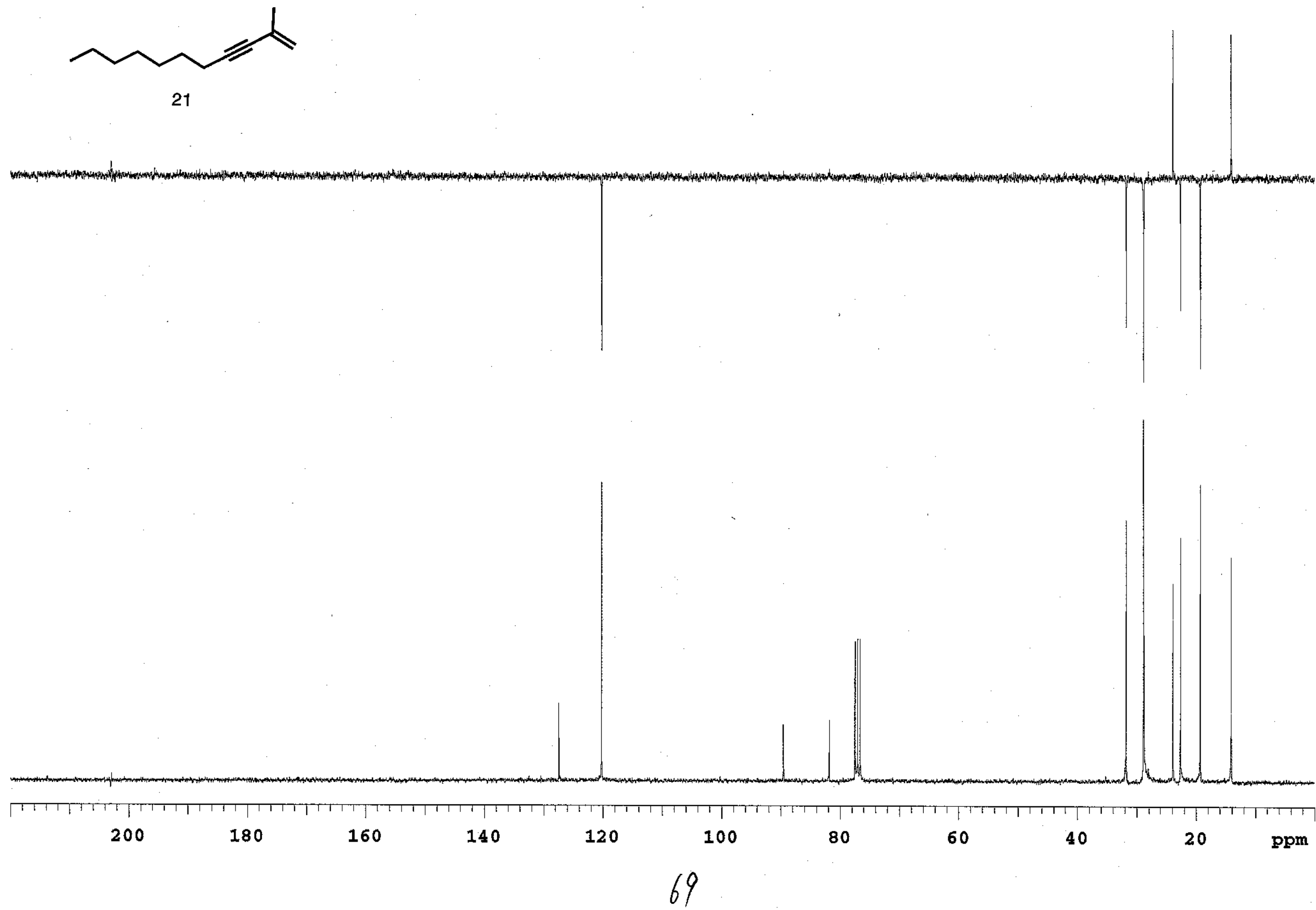


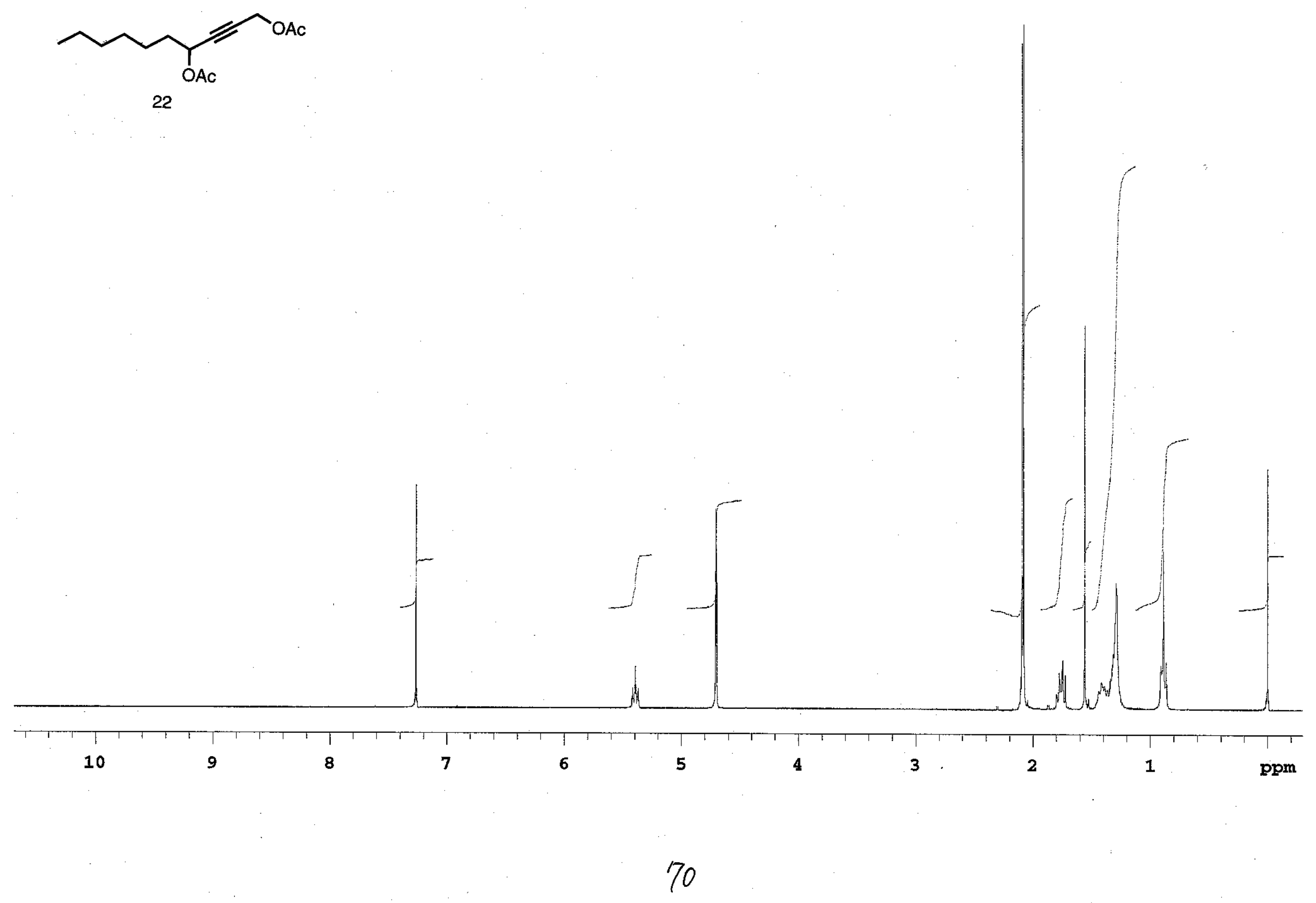




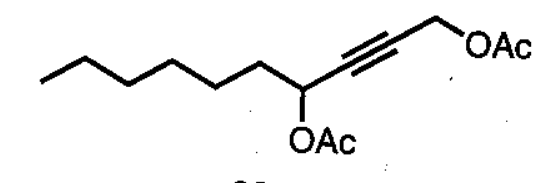

22

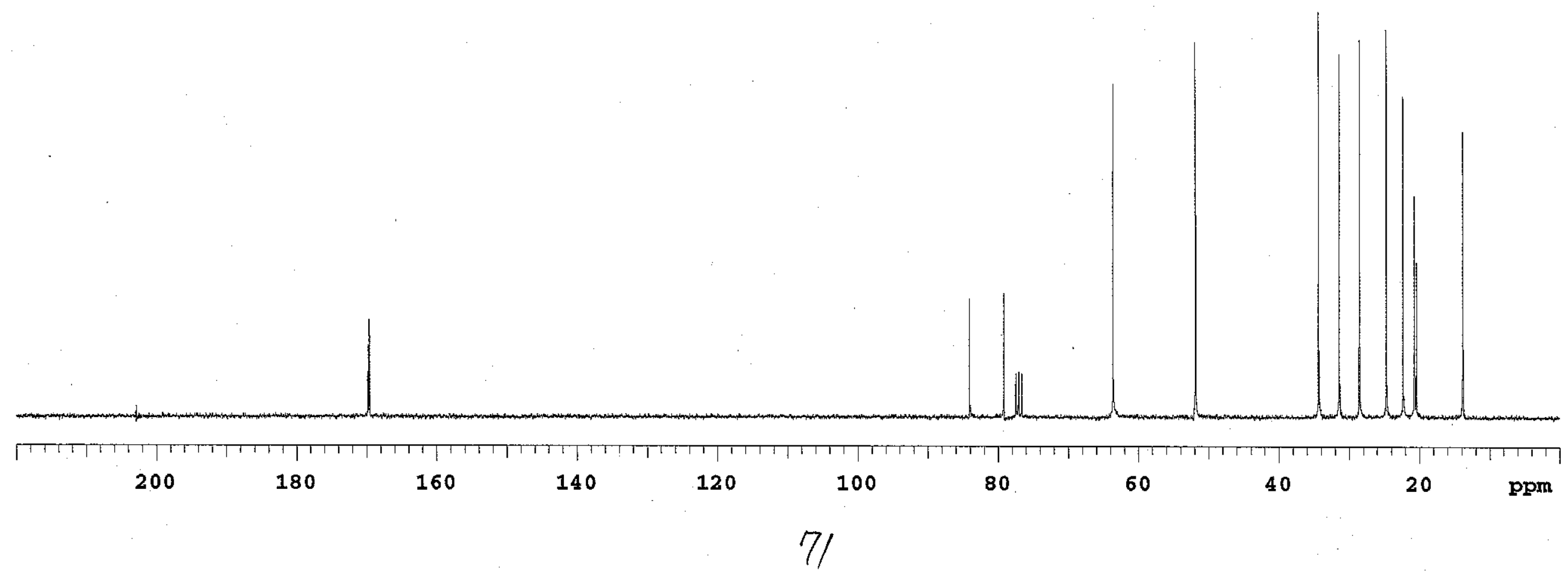




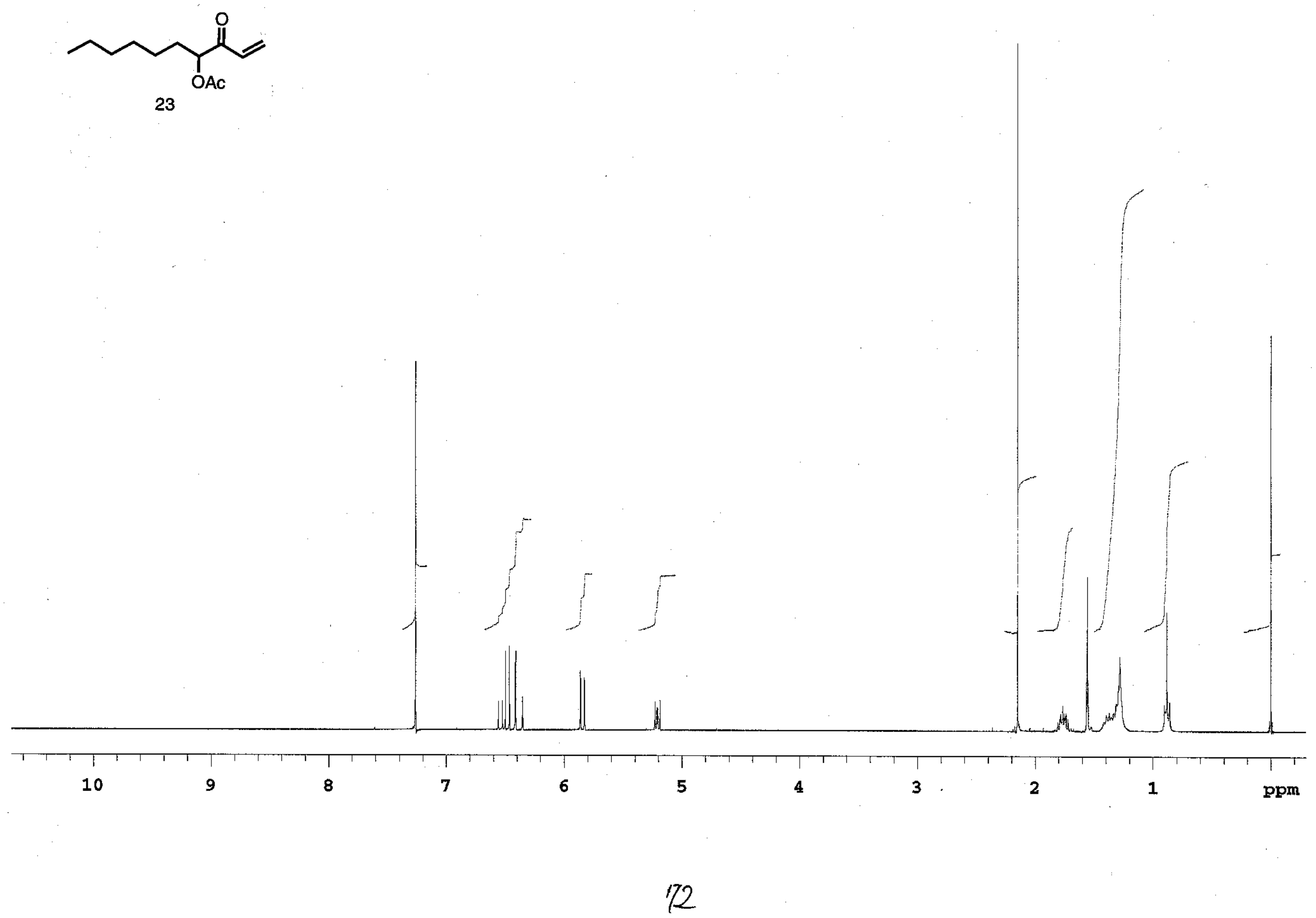




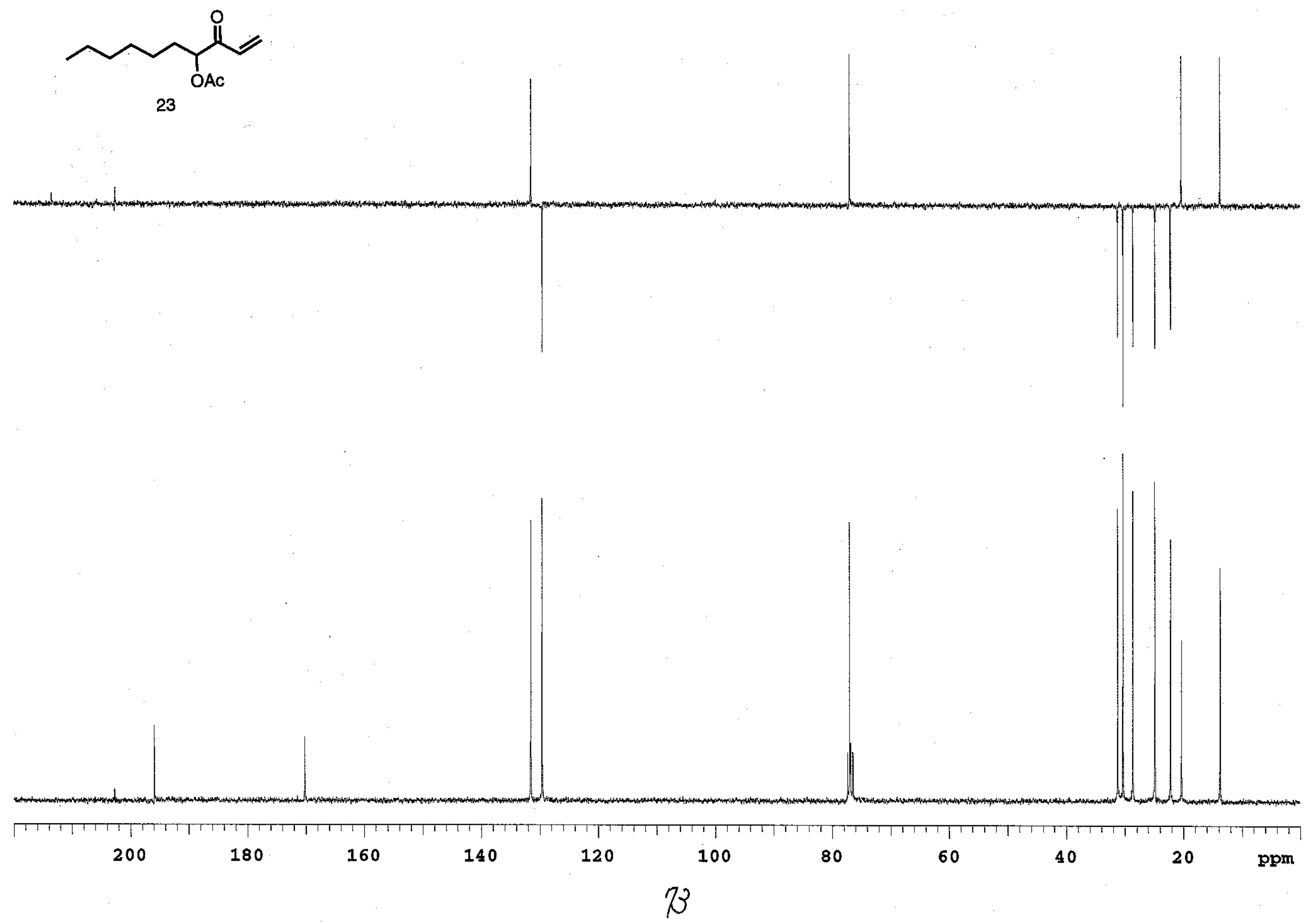




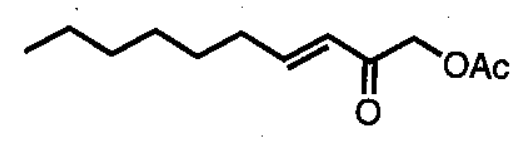

24

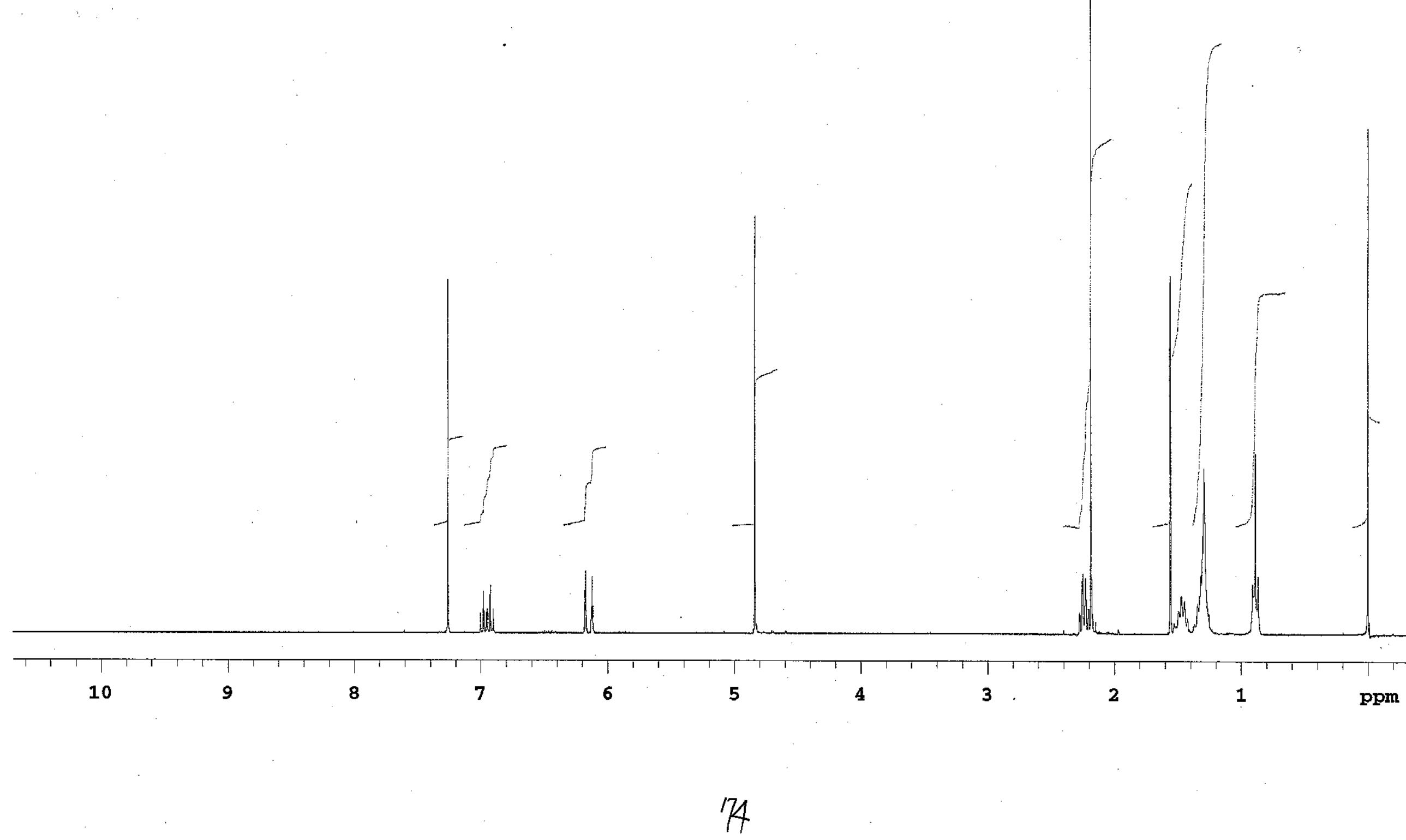




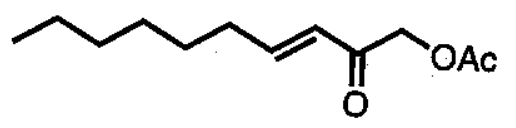

24

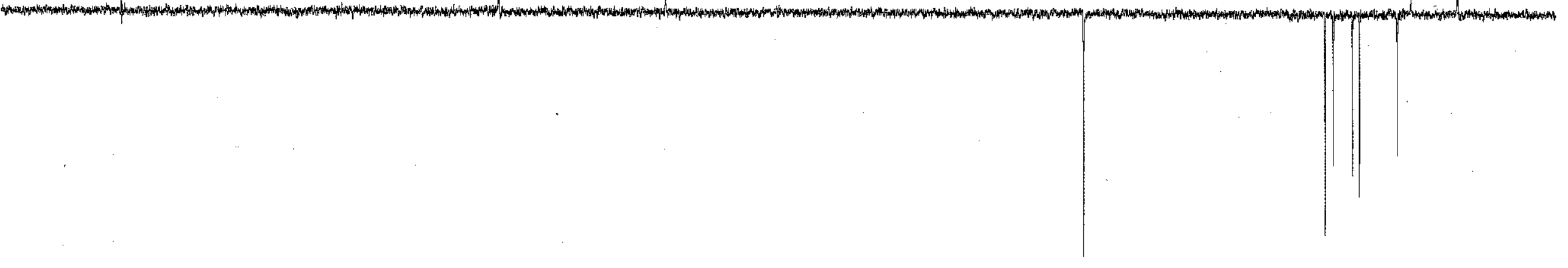

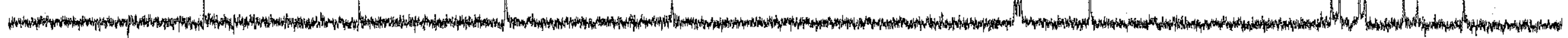

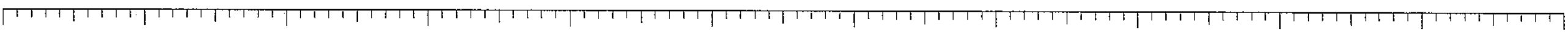



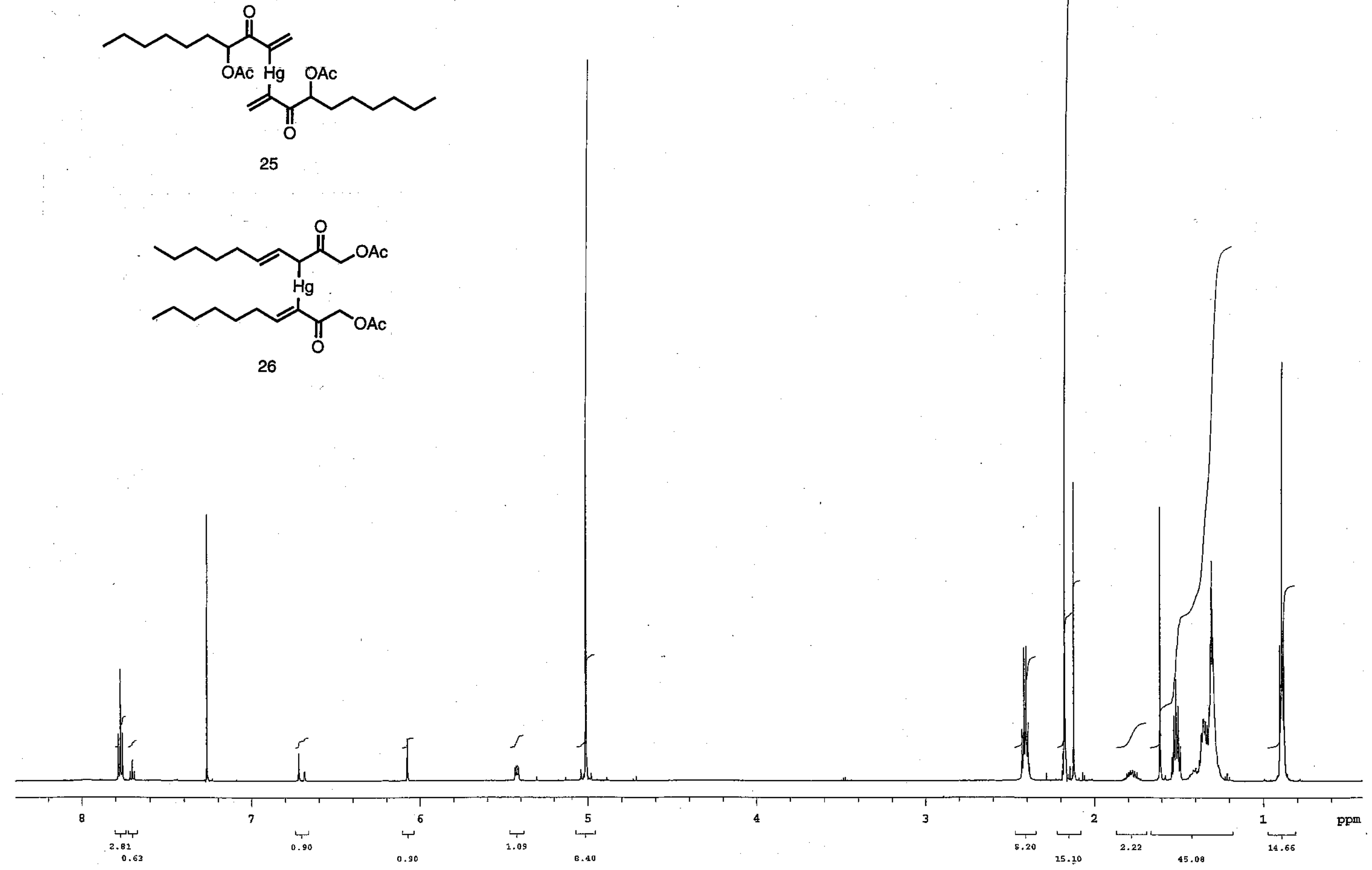